\title{
Climate change and dengue transmission in Vietnam: an integrated assessment
}

Citation for published version (APA):

Toan, D. T. T. (2015). Climate change and dengue transmission in Vietnam: an integrated assessment. [Doctoral Thesis, Maastricht University]. Datawyse / Universitaire Pers Maastricht.

https://doi.org/10.26481/dis.20151209dt

Document status and date:

Published: 01/01/2015

DOI:

$10.26481 /$ dis.20151209dt

Document Version:

Publisher's PDF, also known as Version of record

\section{Please check the document version of this publication:}

- A submitted manuscript is the version of the article upon submission and before peer-review. There can be important differences between the submitted version and the official published version of record.

People interested in the research are advised to contact the author for the final version of the publication, or visit the DOI to the publisher's website.

- The final author version and the galley proof are versions of the publication after peer review.

- The final published version features the final layout of the paper including the volume, issue and page numbers.

Link to publication

\footnotetext{
General rights rights.

- You may freely distribute the URL identifying the publication in the public portal. please follow below link for the End User Agreement:

www.umlib.nl/taverne-license

Take down policy

If you believe that this document breaches copyright please contact us at:

repository@maastrichtuniversity.nl

providing details and we will investigate your claim.
}

Copyright and moral rights for the publications made accessible in the public portal are retained by the authors and/or other copyright owners and it is a condition of accessing publications that users recognise and abide by the legal requirements associated with these

- Users may download and print one copy of any publication from the public portal for the purpose of private study or research.

- You may not further distribute the material or use it for any profit-making activity or commercial gain

If the publication is distributed under the terms of Article $25 \mathrm{fa}$ of the Dutch Copyright Act, indicated by the "Taverne" license above, 


\section{Climate Change and Dengue}

\section{Transmission in Vietnam:}

an Integrated Assessment

Do Thi Thanh Toan 
This research was supported by an educational development grant from the Netherlands Organisation for International Cooperation in Higher Education (EP-Nuffic)

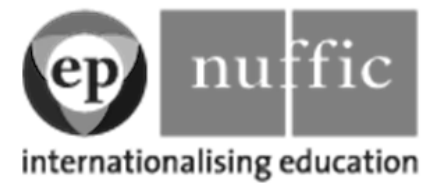

Print: Datawyse - Universitaire Pers Maastricht ISBN: 9789461595058 


\section{Climate Change and Dengue}

\section{Transmission in Vietnam:}

\section{an Integrated Assessment}

\section{DISSERTATION}

to obtain the degree of Doctor at Maastricht University, on the authority of the Rector Magnificus, Prof. dr. L.L.G. Soete, in accordance with the decision of the Board of Deans,

to be defended in public on Wednesday 9 December 2015, at 10.00 hours

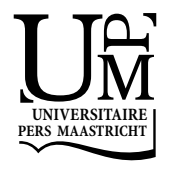




\section{Promotor}

Prof. Dr. Pim Martens

Co-promotors

Ass. Prof. Dr. Luu Ngoc Hoat, Hanoi Medical University, Vietnam

Prof. Dr. E. Pamela Wright, Amsterdam

\section{Assessment Committee}

Prof. Dr. Ralf Peeters - Chairman

Dr. Maud Huynen

Prof. Dr. Thomas Kraft

Prof. Dr. Chris Thomas (Aberystwyth University)

Prof. Dr. Vu Sinh Nam (Vietnam National Institute of Hygiene and Epidemiology) 


\section{Table of Contents}

List of Figures $\quad$ vi

List of Tables viii

1 Introduction 1

1.1 Introduction . . . . . . . . . . . . . . . . . 2

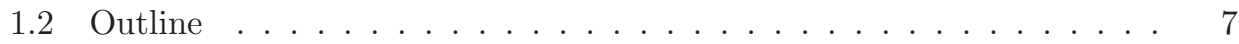

2 Climate change and Dengue Transmission: Evidence and Implica$\begin{array}{ll}\text { tions for Vietnam } & 10\end{array}$

2.1 Climate change in Vietnam . . . . . . . . . . . . . . . . 11

2.1.1 What is climate change? . . . . . . . . . . . . . . 11

2.1.2 How does climate change affect Vietnam? . . . . . . . . . . . . 12

2.1.3 The burden of dengue fever in the world and in Vietnam . . . 16

2.1.4 Dengue vector surveillance and control in Vietnam . . . . . . . 20

3 Conceptual and methodological framework $\quad 22$

3.1 Climate change and vector-borne disease: a framework for analysis . . 23

3.2 Knowledge gap in analyzing dengue fever-climate change relationships 25

3.3 Role of Integrated Assessment . . . . . . . . . . . . . . . . . . 27

3.3.1 Research approach and methods . . . . . . . . . . . 28 
3.3.2 Participatory methods . . . . . . . . . . . . . 30

3.3.3 Analysis of quantitative data . . . . . . . . . . . . 31

3.3.4 Study limitations . . . . . . . . . . . . . . . 33

4 Climatic-driven seasonality of emerging dengue fever in Hanoi, Viet$\begin{array}{ll}\text { nam } & 35\end{array}$

4.1 Introduction . . . . . . . . . . . . . . . . . 36

4.2 Materials and Methods . . . . . . . . . . . . . . . . . . . 39

4.2.1 Study setting . . . . . . . . . . . . . . . . 39

4.2.2 Data collection . . . . . . . . . . . . . . . . 39

4.2 .3 Vapor pressure . . . . . . . . . . . . . . . . 40

4.2.4 Wavelet analysis ... . . . . . . . . . . . 40

4.2.5 Reproductive ratio .................... 41

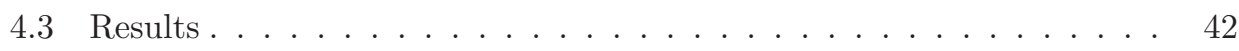

4.3.1 Dengue incidence and its reproductive ratio in Hanoi . . . . . . 42

4.3.2 Meteorological variables in Hanoi . . . . . . . . . . . . . . . . 44

4.3.3 Coherences between meteorological variables and DF incidence in Hanoi . . . . . . . . . . . . . . . . . . . . . 44

4.4 Discussion and conclusion . . . . . . . . . . . . . . 47

5 Risk factors associated with an outbreak of dengue fever/dengue haemorrhagic fever in Hanoi, Vietnam 53

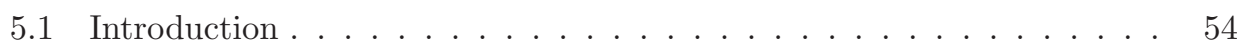

5.2 Materials and methods . . . . . . . . . . . . . . . . 54

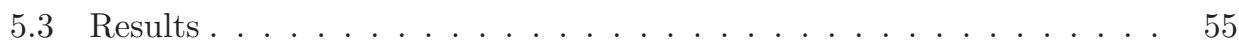

5.4 Discussion . . . . . . . . . . . . . . . . . . . 58

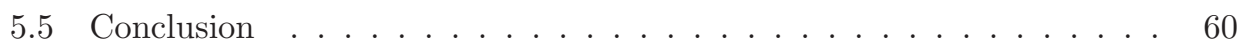


6 Environmental factors influencing epidemics of dengue fever in Vietnam

6.1 Introduction . . . . . . . . . . . . . . . . . . . . 63

6.2 Methodology . . . . . . . . . . . . . . . . . . 64

6.2 .1 Study site . . . . . . . . . . . . . . . . . 64

$6.2 .2 \quad$ Data sources . . . . . . . . . . . . . . . . 65

6.2 .3 Data analysis . . . . . . . . . . . . . . . . . . . 65

6.3 Results. . . . . . . . . . . . . . . . . 67 67

6.3.1 Distribution of cases in relation to distance from a body of water 67

6.3.2 Distance of cases from nearest roads . . . . . . . . . . . . . 69

6.3.3 Risk factors for appearance of dengue cases per commune . . . 69

6.4 Discussion . . . . . . . . . . . . . . . . . 72

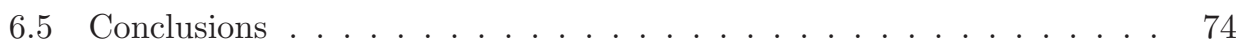

7 Hot spot detection and spatiotemporal dispersion of dengue fever in $\begin{array}{ll}\text { Hanoi, Vietnam } & 76\end{array}$

7.1 Introduction . . . . . . . . . . . . . . 77

7.2 Method ......................... 77

7.2.1 Some features of Hanoi . . . . . . . . . . . . . 77

$7.2 .2 \quad$ Data analysis . . . . . . . . . . . . . . . . . . 79

7.3 Results. . . . . . . . . . . . . . . . . . 80

7.3 .1 Descriptive analysis $\ldots \ldots \ldots \ldots$. . . . . 80

7.3.2 Spatial autocorrelation of dengue fever . . . . . . . . 84

7.3.3 Purely temporal clustering _ . . . . . . . . . . . . 84

7.3 .4 Purely spatial clustering . . . . . . . . . . . . . . 85

7.3.5 Space-Time Clustering . . . . . . . . . . . . . 85

7.3 .6 Dynamic dispersion of DF . . . . . . . . . . . 86

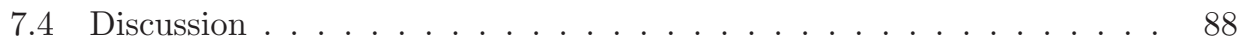


8 Perceptions on climate change and its impact on human health $\quad 90$

8.1 Introduction . . . . . . . . . . . . . . . . . 91

8.2 Methods . . . . . . . . . . . . . . . . . . 92

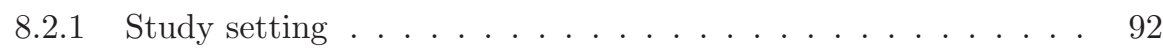

8.2.2 Study design and participants . . . . . . . . . . . 92

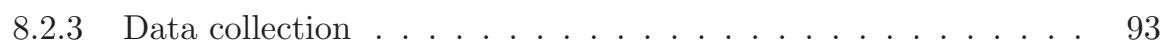

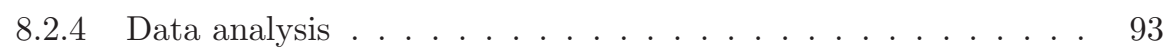

8.2.5 Ethical considerations ................... 94

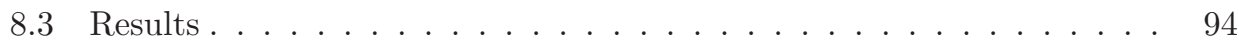

8.3.1 Perception of climate change ... . . . . . . . . . 94

8.3.2 Perception of impacts of climate change and social determinants 98

8.4 Discussion . . . . . . . . . . . . . . . . . . 99

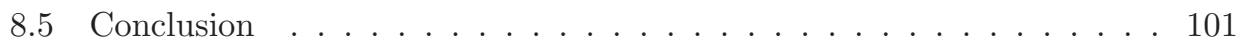

8.6 Policy recommendations . . . . . . . . . . . . . . . . 102

9 Discussion and Conclusion $\quad 103$

9.1 Potential effect of climate change on dengue transmission . . . . . . 105

9.2 Potential for change in dengue fever patterns . . . . . . . . . . . 108

9.2.1 Climate change and human health: public perceptions . . . . . 109

9.2.2 Future perspectives . . . . . . . . . . . . . 111

Bibliography

$\begin{array}{ll}\text { Summary } & 133\end{array}$

$\begin{array}{lr}\text { Samenvatting } & 139\end{array}$

$\begin{array}{lr}\text { Valorisation } & 144\end{array}$

$\begin{array}{lr}\text { Acknowledgements } & 147\end{array}$ 


\section{List of Figures}

2.1 Distribution of dengue types in Vietnam from 1991 to 2011. . 19

3.1 Research assessment framework . . . . . . . . . . . . . . . 34

4.1 The reproductive ratio, time series and wavelet power spectrum of DF in Hanoi (14 districts) from 2002 to 2009. . . . . 43

4.2 Time series and wavelet power spectra of mean temperature, cumulative rainfall and mean absolute and relative humidities in Hanoi, from 2002 to 2009.

4.3 Cross-correlation between the 4 climatic variables: mean temperature, rainfall, vapor pressure and relative humidity . . . 46

4.4 Cross-wavelet power spectra between DF and mean temperature, rainfall, absolute and relative humidities in Hanoi from 2002 to 2009

6.1 Frequency distribution of the case water body distance for

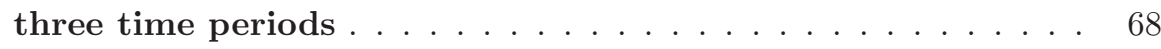

6.2 Dengue cases close to main roads . . . . . . . . . . . 70

6.3 Maps of dengue incidence rate, population density, street length area ratio and water surface area ratio $\ldots \ldots \ldots 71$ 
7.1 Location of the study area Hanoi, Vietnam . . . . . . . . . 78

7.2 Numbers of dengue cases and postcode areas with dengue notifications between January 2004 and December 2009 in Hanoi ............................ 81

7.3 Boxplots of the seasonal distribution of numbers of postcode areas with dengue infection in three periods in Hanoi . . . . . 82

7.4 Map showing raw dengue incidence rates in three periods . . 83

7.5 Scatterplot of significant space-time clusters in Hanoi from

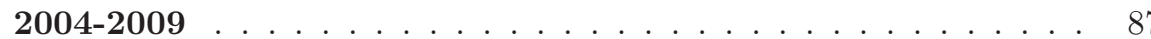




\section{List of Tables}

2.1 Projected increase in annual average temperatures relative to 1980-99 (MONRE Medium Scenario, $\left.{ }^{\circ} \mathrm{C}\right)$ Climatic zone. . . . . . . . . . . . . . 13

2.2 Projected changes in annual rainfall relative to 1980-99 (MONRE Medium Scenario, Climatic zone. . . . . . . . . . . . . . . . . . 14

5.1 Demographic characteristics of dengue fever/dengue haemorrhagic fever (DF/DHF) and control patients Characteristics. . . . . . . . . 56

5.2 Housing variables of dengue fever/dengue haemorrhagic fever (DF/DHF) and control patients Variables. . . . . . . . . . . . . . . 57

5.3 crude and adjusted logistic regression odds ratios on selected variables. 58

6.1 Total number of cases according to years. . . . . . . . . . . . . . 66

6.2 Distance of cases from water bodies for three time periods. . . . . . . 67

6.3 Cases per $\mathrm{km}^{2}$ close to water bodies. . . . . . . . . . . . . . 68

6.4 Cases per $\mathrm{km}^{2}$ close to roads. . . . . . . . . . . . . . . . . . 69

6.5 Ordinary Least Squares (OLS) results. . . . . . . . . . . . . . . 72

7.1 Descriptive statistics of monthly numbers of postcode areas with notified dengue cases. . . . . . . . . . . . . . . . 81

7.2 Spatial autocorrelation analysis for dengue in Hanoi, 2004-2009. . . . . 84 
7.3 The clusters of dengue cases detected using the purely temporal analysis. 84

7.4 The clusters of dengue cases detected using the purely spatial analysis. 85

7.5 SaTScan statistics for space-time clusters with significantly higher incidence in Hanoi from 2004-2009 (Most likely cluster). . . . . . . . . . 85

7.6 Changes of dengue fever in latitude and longitude, Hanoi, 2004-2009. . 86

8.1 Demographic characteristics of study population $(\mathrm{n}=1412$ people). . . 95

8.2 Perceptions of climate change and its impact. . . . . . . . . . . . . 96

8.3 Perceived changes in heat and cold compare to five years ago $(n=1412) .97$

8.4 Associations between perception of the impact of climate change and social determinants . . . . . . . . . . . . . . . . . 99 
Chapter 1

\section{Introduction}




\section{INTRODUCTION}

\subsection{Introduction}

Climate change has already been observed globally, including increases in air and water temperatures, reduced number of frost days, increased frequency and intensity of heavy downpours, a rise in sea level, and reductions in snow cover, glaciers, permafrost, and sea ice (WHO, 2009). In 1990, the Intergovernmental Panel on Climate Change (IPCC) concluded that climate change is human-induced (Thornes, 2002). This conclusion was repeated in the Framework Convention on Climate Change (UNFCCC) in Article 1, which defines climate change as: a change of climate which is attributed directly or indirectly to human activity that alters the composition of the global atmosphere and which is in addition to natural climate variability observed over comparable time periods. In the 2014 report of IPCC on March, the effects of climate change are reported to be occurring on all the continents and to have an impact on natural and human systems. According to this report, changing precipitation or melting snow and ice are significantly affecting the availability and quality of water resources in many areas. In the agriculture sector, the negative impacts of climate change are resulting in lower crop yields and growth rates, and a loss of soil fertility. Furthermore, climate-related extremes, such as heat waves, droughts, floods, cyclones, and wildfires result in significant alteration of ecosystems and increasing vulnerability of human systems. Due to global warming, there has been an increase in heat-related mortality and a decrease in cold-related mortality. In addition, local changes in temperature and rainfall have altered the distribution of many vector-borne and water-borne diseases (IPCC, 2014).

The link between population health and climate change has been demonstrated by scientists who warned that climate change poses a wide range of risks to population health. As noted by the World Health Organization (WHO), climate change is not just a threat to biological systems and the environment but a significant and emerging threat to public health, especially in lower income populations and tropical/subtrop- 


\section{INTRODUCTION}

ical countries. Protecting health from the impacts of climate change is recognized as one of the defining challenges in this century (WHO, 2009).

Three types of health repercussions from climate change have been recognized by the World Health Organization (WHO, 2003): (1) direct effects of extreme climate events; (2) health consequences of environmental disorders due to climate change; and (3) other consequences on health (i.e. trauma, infections, psychological conditions, and negative effects on food security, among others) caused by populations being displaced due to economic problems, environmental degradation or conflicts arising because of climate change. The Fourth Assessment Report of the Inter-governmental Panel for Climate Change (IPCC) highlights the substantial improvement in our understanding of the health problems resulting from climate change since the previous reports (IPCC, 2007). In many developed countries, significant results were gained in the national health impact assessments of climate change (Costello et al., 2009; Ford and Furgal, 2009; Ford, 2012; Huynen et al., 2001; Kovats et al., 2003; Patz et al., 2000; Watts, 2010). For example, Huynen et al. (2001) conducted a study on the mortality effects of climate change. They combined observed mortality risks accompanying temperature change, with the projected changes in monthly temperature estimated by nine General Circulation Models for the year 2050 under the IPCC 550 ppm stabilization scenario (i.e. approximate doubling of the pre-industrial level of greenhouse gases), in order to estimate future changes in mortality rates. Their findings showed that for the Netherlands, the continuing decreasing trend in winter mortality is more pronounced than the increasing excess summer mortality rate. Net yearly decreases in mortality rates varied between 0.9 and $1.6 \%$ for cardiovascular mortality, between 1.6 and $3.9 \%$ for respiratory mortality, and between 0.6 and $1.1 \%$ for total mortality. However, in developing countries, only a few studies have been done on these issues (Berrang-Ford et al., 2011; Bultó et al., 2006; Ebi et al., 2006; Friel et al., 2008; Hajat and Kosatky, 2010). One example used empirical statistical 


\section{INTRODUCTION}

methods to elaborate a special index to facilitate the description of the association between the weather, climate variability and health outcomes in Cuba, which can be applied to predict outbreaks of some climate-sensitive diseases (Bultó et al., 2006). Another study conducted in several Pacific small island states pointed out the changes in the distribution and burden of climate-sensitive diseases in the light of regional climate pattern changes (Ebi et al., 2006).

Links between diseases and the present climate have been found to be strongest between climate and mosquito borne diseases (Bouzid et al., 2013; Ebi et al., 2005; Myers and Patz, 2009; Small et al., 2003). Scientific interest in the relationship between infectious diseases and climate change has risen since the mid-1980s. Infectious disease transmission is influenced by many extrinsic factors (such as climatic and environmental factors, socio-economic conditions, health services and interventions) as well as intrinsic factors (such as population immunity). Many infectious agents, vector organisms, and reservoir host species, as well as rates of pathogen replication within vector organisms, are sensitive to climatic conditions (Kovats et al., 2003; Mai Kien et al., 2010; McMichael and Butler, 2006; Semenza and Menne, 2009). For example, in regions where low temperature, low rainfall or lack of vector habitat limit vector-borne disease transmission (including malaria), climate change may alter the ecological balance and trigger epidemics. Epidemics can also result from climaterelated migration of reservoir hosts or human populations (WHO 2006). In the case of dengue fever (DF) disease, climate affects the dengue virus and vector populations both directly and indirectly (Arcari et al., 2007; Gubler et al., 2001; Kearney et al., 2009; Martens et al., 1995b; Thai and Anders, 2011; Tseng et al., 2009). Temperature influences vector development rates, mortality, and behavior (Kearney et al., 2009; Martens et al., 1995b; Rueda et al., 1990; Tun-Lin et al., 2000) as well as controlling viral replication within the mosquito (Watts et al., 1987). Variability in precipitation influences habitat availability for the vectors - Aedes aegypti and Aedes albopictus 


\section{INTRODUCTION}

larvae and pupae. Temperature further interacts with rainfall as the chief regulator of evaporation, thereby also affecting the availability of water habitats. Indirectly, rainfall, temperature, and humidity influence land cover and land use, which can promote or impede the growth of vector populations. The incidence of DF has been associated with vegetation indices, housing quality, and surrounding land cover (Chadee et al., 2007; Tseng et al., 2009; Van Benthem et al., 2005). Climate change can also alter how humans interact with the land, altering its use and therefore impacting the magnitude of the mosquito population as well as species composition (Chang et al., 1997; Vanwambeke et al., 2007).

Among the many developing countries, Vietnam is one of those most affected by climate change (DANIDA, 2005). The country suffers from many kinds of natural disasters; annually, an estimated one million Vietnamese are affected by them. In addition, Vietnam will suffer the growing impact of natural disasters caused by climate change, especially storms and floods which are increasing in frequency and intensity.

The impact of climate change on individuals varies according to diverse factors including age, occupation, gender, education and economic status (Banu et al., 2011; Be et al., 2007; Few and Tran, 2010). Factors that influence peoples concern about climate change include past experiences of the impact of flooding on livelihood and observation of changes in the climate (MOH, 2007).

With the approval by the Government and the starting of nation-wide implementation of the National Target Program for addressing Climate Change (the current national climate change strategy for Vietnam), the Vietnam Ministry of Health is facing challenges in developing a Climate Change Action Plan for the health sector at the central, subnational and provincial levels. There is a crucial need for integrating climate change issues into health strategies and policies, making them climate proof, as well as for research into health implications (impacts and risks) of climate factors and the health-climate linkage. 


\section{INTRODUCTION}

Although many studies have been carried out to identify the impacts of climate change on the epidemiology and impacts of dengue fever in different geographical locations, there is still a need for a more comprehensive and quantitative evaluation of these impacts, certainly for Vietnam a country that ranks near the international top in terms of high morbidity for dengue fever in the sub-tropical region. The country also suffers from many kinds of natural disasters that could mediate the link between climate change and human health problems. Currently, in Vietnam, climate change and disaster issues have not yet been integrated into health policies, strategies and plans, partly because of the weak linkages between policy development and scientific evidence, and partly due to the lack of reliable quantitative and qualitative data on the topic. For example, in the Vietnam National Strategy on Preventive Medicine and Master Plan on Health Care System Development (MOH, 2007), only a few words in one paragraph mentioned climate and environmental change and its consequences. There is no text about potential impact or vulnerability assessment plans or adaptation and response programs that should be considered for the health sector.

The studies in this thesis assist in understanding these complex links as well as understanding peoples concerns, and in quantifying the relationships between socioecological factors (climate variables, mosquito density, vegetation and human population) and the epidemic potential of DF infection in Hanoi, Vietnam. We contribute to the growing knowledge base on the assessment of potential impact of socioenvironmental change upon the transmission of DF infection. Increased understanding of the relative importance of social and ecological variables in the transmission cycles of DF will aid public health planning and policy-making to develop effective strategies to control and prevent this increasingly wide-spread disease. Epidemic forecasting models were developed which may be used directly for the decision-making processes in the surveillance and control of DF disease. Additionally, the methods developed through this study may have a wider application to other public health 


\section{INTRODUCTION}

problems which have comparable elements in their epidemiology.

Based on the background sketched above, the central question this thesis aims to answer is:

In what different ways might climate change affect dengue fever transmission in the specific context of Hanoi?

In order to address this question, the following research objectives were identified:

1. To identify any direct and indirect link between climatic variables and DF incidence in Hanoi, Vietnam,

2. To estimate the potential a future change of dengue fever pattern in Hanoi,

3. To describe the perceptions on climate change and its impact on human health among people in Hanoi.

\subsection{Outline}

The thesis is structured as follows: the next chapter (Chapter 2) reviews the literature to provide background information on dengue fever and climate change, with specific reference to the city of Hanoi. A thorough knowledge of the current situation of climate change and of the burden of dengue fever in Vietnam is necessary before the methodology can be presented.

Chapter 3 presents the methodological framework for analysis. In this chapter, we discuss how Integrated Assessment (IA) is used as an overarching research philosophy in this thesis. The combination of different tools and research methods is needed to assess such a complex transmission as that of dengue.

Climate change would not affect human health in isolation, but in conjunction with other ecological and demographic changes. The reports presented in Chapters 4 to 7 help to identify whether there are any conditions resulting from climate change 


\section{INTRODUCTION}

that directly or indirectly affect the transmission of dengue fever. Chapter 4 describes the use of the wavelet analysis method to detect and measure the seasonality of DF and to investigate its direct links to climatic variables. We identified which among four climatic variables - vapor pressure and relative humidities, temperature and rainfall - are most critical for the onset of dengue epidemics. Chapter 5 explores the risk factors associated with DF in patients admitted to hospitals in Hanoi. The demographic characteristics of the patients such as age, education, occupation and the variables related to their housing conditions were included in the multivariable backward stepwise logistic regression model to identify key risk factors for DF. Moreover, as the risk of dengue fever is known to vary with space and time, we need precise knowledge of the risk factors, the risk localities, the levels of risk, and details on the exposed population. Chapter 6 applied GIS technique to map the risk of DF with selected environmental factors such as surface hydrology and vegetation. Spatial scan statistics was applied in Chapter 7 to detect any hot spots in Hanoi and to identify the dynamics of the dispersion of DF.

Results from Chapters 4 to 7 also provide estimation of potential changes in the patterns of dengue fever in the future. In Chapter 4, we estimate the values for the reproductive ratio of dengue fever through time and characterize the trend and seasonality of the reproductive ratio. Then, we discuss these results in the light of immunological and entomological factors specific to dengue epidemiology in Hanoi City. Chapter 5 and Chapter 6 report on the measure of the correlations between socio-demographic factors that could affected dengue fever, and Chapter 7 presents the estimates of how spatial patterns may change over time for DF in Hanoi.

Chapter 8 reports the perceptions of people in Hanoi about climate change and the impact of climate change on human health. Appreciating the complex relations between climate change and human health as well as awareness of peoples concerns form the basis for policy makers to develop communication strategies to engage com- 


\section{INTRODUCTION}

munities most effectively.

Chapter 9 summarizes the study findings across the five chapters, and reviews conclusions in relation to the overall aims of the study. We first discuss how people become aware about climate change and human health, and link that to the case of dengue fever in Hanoi. We then discuss the direct and indirect impacts of climate change on dengue fever transmission, taking into account the case of dengue fever in Hanoi as an example for intepreting the case of dengue fever at regional and global levels. Finally, we discuss the future changes in dengue transmission patterns based on findings from research. We will also provide an overview of estimation for global climate change and human health from the point of view of dengue cases. This Chapter 9 further notes the study limitations, presents the public health implications of the results, and proposes directions for future research. 
Chapter 2

Climate change and Dengue

Transmission: Evidence and

Implications for Vietnam 


\section{CLIMATE CHANGE AND DENGUE TRANSMISSION: EVIDENCE AND IMPLICATIONS FOR VIETNAM}

\subsection{Climate change in Vietnam}

\subsubsection{What is climate change?}

Climate change is defined as any long-term significant change in the average weather that a given region experiences. In 1992, at the Earth Summit in Rio de Janeiro, the United Nation Framework Convention on Climate Change (UNFCCC) stated that Human activities have substantially increased the global atmospheric concentration of greenhouse gas. These increases enhance the natural greenhouse effect, which results in warming of the earths surface and atmosphere and may adversely affect natural ecosystems and humankind.

According to the fourth assessment report of the Intergovernmental Panel on Climate Change (IPCC, 2007), the expected increase in world average temperature by 2100 is within the range $1.4-5.8^{\circ} \mathrm{C}$, becoming greater at higher latitudes and over land. Another report, from the American Meteorological Society, recently gave an estimation that global temperatures will rise by 3.5 to $7.4^{\circ} \mathrm{C}$ in less than one hundred years (Herring et al., 2014). Furthermore, the global average annual rainfall will increase. In some regions precipitation events and flooding could become more severe, while many mid-latitude and lower latitude land regions will become drier. Climate variability is expected to increase in a warmer world (IPCC, 2014). Global climate change is already an imminent threat and the projected changes in the earth's climate are an environmental concern with severe social and economic implications. Climate change represents a serious challenge to the three pillars of sustainable development - economics, society, and the environment. In the agriculture sector, climatic stresses may result in lower crop productivity, the need to change cropping systems, and weaker resistance to infectious diseases. The 2014 report from the IPCC shows that negative effects of climate change on crop yields have been more common than positive impacts and that the greatest effect was found on wheat and maize yields. 


\section{CLIMATE CHANGE AND DENGUE TRANSMISSION: EVIDENCE AND IMPLICATIONS FOR VIETNAM}

Climate change may lead to more frequent and intense natural disasters such as typhoons, floods, droughts and saline water intrusion, all of which are causes of declines in national food output and cultivable land.

Regarding the society, climate change is likely to exacerbate poverty and have a negative impact on human health; it may also shorten life expectancy. Climaterelated hazards have directly affected the livelihoods of poor people or destroyed their homes, or indirectly affected them by increasing food prices and food insecurity. In addition, climate change could lead to greater likelihood of injury, disease, and death due to more intense heat waves and to increased likelihood of malnutrition because of reduced food production in poor regions. Increasing numbers of outbreaks of infectious diseases such as malaria, dengue and a number of fatal viruses are also expected. With regard to environmental issues, climate change may reduce the richness of biodiversity and eco-systems, and make the management of water resources and forests an even greater challenge.

\subsubsection{How does climate change affect Vietnam?}

Vietnam is a long narrow country with an extensive coastline, two major river deltas, and mountainous areas on its eastern and northeastern borders. Vietnam is heavily exposed to the risks of weather variability and climate change. In a study of eighty-four countries, Vietnam was ranked among those that can expect the most adverse impacts of global climate change and especially sea level rise, affecting land, population, GDP, urbanization, agriculture, and wetlands (Dasgupta et al., 2009). The IPCCs Fourth Assessment characterized Vietnam as a natural disaster hotspot, ranking 7 th globally on economic risk, 9th on land area and population exposed, and 22nd on mortality from multiple hazards.

In June 2009, the Ministry of Natural Resources and Environment of Vietnam (MoNRE) published Vietnams official scenario for climate change. While climate 


\section{CLIMATE CHANGE AND DENGUE TRANSMISSION: EVIDENCE AND IMPLICATIONS FOR VIETNAM}

change estimates were developed for three different emissions scenarios: low (B1), medium (B2), and high (A2 and A1FI), the medium emission scenario (B2) was selected by MoNRE for the purpose of impact assessment and adaptation planning. The official scenario includes projected changes in temperature, rainfall, and sea level over the period 2020 to 2100 (MONRE, 2009).

Temperature According to MONRE (2009), the annual average temperature in Vietnam increased by 0.5 to $0.7^{\circ} \mathrm{C}$ over the period $1958-2007$. The report further notes that winter temperatures have increased faster than summer temperatures, and that temperatures in Northern Vietnam have increased faster than those in the South. Annual average temperatures observed in Hanoi, Danang, and Ho Chi Minh City have all been higher over the decade 19912000 than the 193140 decade, and still higher in 2007 than over the 19912000 decade. These observations are all consistent with measured increases in global average temperature. The projected increases in average temperature are slightly higher in the sub-temperate Northern part of the country $\left(2.46 .8^{\circ} \mathrm{C}\right.$ by 2100$)$ than in the tropical South $\left(1.62 .0^{\circ} \mathrm{C}\right)$ (Table 2.1$)$

Table 2.1: Projected increase in annual average temperatures relative to 1980-99 (MONRE Medium Scenario, ${ }^{\circ} \mathrm{C}$ ) Climatic zone.

\begin{tabular}{llllll}
\hline Climatic zone & 2020 & 2040 & 2060 & 2080 & 2100 \\
\hline North-West & 0.5 & 1.0 & 1.6 & 2.1 & 2.6 \\
\hline North-East & 0.5 & 1.0 & 1.6 & 2.1 & 2.5 \\
\hline North Delta & 0.5 & 0.9 & 1.5 & 2.0 & 2.4 \\
\hline North-Central & 0.5 & 1.1 & 1.8 & 2.4 & 2.8 \\
\hline South-Central & 0.4 & 0.7 & 1.2 & 1.6 & 1.9 \\
\hline Central Highlands & 0.3 & 0.6 & 1.0 & 1.4 & 1.6 \\
\hline South & 0.4 & 0.8 & 1.3 & 1.8 & 2.0 \\
\hline
\end{tabular}

Precipitation As noted in the official scenario (MONRE, 2009), changes in rainfall patterns are complex, seasonal, and region-specific. Over the last century, changes in annual average rainfall were not systematically either upward or downward. Periods with declining rainfall were followed by periods with increasing rainfall. However, the 


\section{CLIMATE CHANGE AND DENGUE TRANSMISSION: EVIDENCE AND IMPLICATIONS FOR VIETNAM}

annual rainfall appears to have decreased slightly over climate zones in the North, and increased over climate zones in the South. Despite the lack of obvious and definite trends in historical data, annual rainfall is projected to increase by $45 \%$ in Northern Vietnam by 2060 and by $78 \%$ by 2100 . The projected changes in southern Vietnam are rather smaller, $1.53 \%$ by 2100 (table: 2.2 )

Table 2.2: Projected changes in annual rainfall relative to 1980-99 (MONRE Medium Scenario, Climatic zone.

\begin{tabular}{lrrrrr}
\hline Climatic zone & 2020 & 2040 & 2060 & 2080 & 2100 \\
\hline North-West & 1.4 & 3.0 & 4.6 & 6.1 & 7.4 \\
\hline North-East & 1.4 & 3.0 & 4.7 & 6.1 & 7.3 \\
\hline North Delta & 1.6 & 3.2 & 5.0 & 6.6 & 7.9 \\
\hline North-Central & 1.5 & 3.1 & 4.9 & 6.4 & 7.7 \\
\hline South-Central & 0.7 & 1.3 & 2.1 & 2.7 & 3.2 \\
\hline Central & 0.3 & 0.5 & 0.9 & 1.2 & 1.4 \\
Highlands & & & & 1.2 & 1.5 \\
\hline South & 0.3 & 0.6 & 1.0 & & \\
\hline
\end{tabular}

Rainfall projections across seasons are of particular interest. The dry seasons are projected to get drier, with the reductions in MarchMay rainfall being higher in the Southern part of the country, while the wet seasons are projected to get wetter, with the JuneAugust rainfall increases being higher in the Northern part of the country. Hence, it is expected that rainfall will be concentrated even more than it is now in the rainy season months, leading to an increase in the frequency, intensity, and duration of floods, and to an exacerbation of drought problems in the dry season. Sea level is projected to rise approximately $30 \mathrm{~cm}$ by 2050 and up to $75 \mathrm{~cm}$ by 2100 under the medium scenario.

Aiming to provide a more comprehensive view of the range of potential outcomes, and given the great uncertainty in all models and scenarios, the modelling performed by World Bank (WB, 2010) or International Food Policy Research Institute (IFPRI, 2011) on climate change in Vietnam generally found the MoNRE scenario to be com- 


\section{CLIMATE CHANGE AND DENGUE TRANSMISSION: EVIDENCE AND IMPLICATIONS FOR VIETNAM}

plementary to other IPCC scenarios. For example, given the uncertainty over climate change impacts on rainfall in Vietnam, in addition to MoNREs scenario, both World Bank and IFRRI have included the driest and wettest scenarios in order to have a better estimation of the potential range of outcomes and impacts. Furthermore, in the response to Vietnams Second Submission to the IPCC in 2011, the United Nations stated that The B2 scenario will lead to an average annual temperature rise in Vietnam by 2100 of about $2.3{ }^{\circ} \mathrm{C} \ldots$ however, recent scientific data suggest that the world is still on a high emissions pathway, and according to the A2 high emissions scenario, the average annual temperature rise would be as much as $3.6{ }^{\circ} \mathrm{C}$ in the North-central coastal region. Under the high emissions scenario, the temperature in the North is estimated to double that increase, while in the South, rainfall would increase in the rainy season (with more extreme rainfall events, flooding and landslides) and decrease by $20 \%$ during dry months (IFAD, 2014).

A large proportion (74\%) of the population of Vietnam is concentrated along the coastal plains and river deltas, which would be the areas most affected by sea level rise (Carew-Reid, 2008). A rise of one meter in sea level, which is likely to be reached sometime in the next century, would directly affect almost $10 \%$ of the current population. The most serious effects of climate change and sea level rise would be increases in flooded areas, obstructions to water drainage, intensified coastline erosion, and enhanced salt water intrusion. These would cause difficulties for agricultural production and domestic water use, and create risks to coastal infrastructure, urban areas and coastal communities. Rises in sea level and sea water temperature will have strong adverse effects on coral reefs and mangrove forests, which are vital to coastal aquaculture and fishery (MONRE/PEP/UNDP, 2008).

The greatest concern of the Vietnamese Goverment currently is climate-related risk of potential negative effects on poverty. McElwee (2010) conducted a study in Vietnam on the social dimensions of adaptation to climate, and found that the di- 


\section{CLIMATE CHANGE AND DENGUE TRANSMISSION: EVIDENCE AND IMPLICATIONS FOR VIETNAM}

rect factor considered by most community members to be among the main causes of poverty was climate change. Drought and hazard damage were brought up again and again as main causes of poverty (McElwee, 2010). In another study conducted in a poor community of a mountainous province, Duong (2009) found that in the 10 years between 1998 and 2008, there were 10 major events causing financial losses, including floods, landslides, cold spells and typhoon-associated storms. Women, ethnic minorities and disabled people are the most vulnerable people and are likely to be disproportionally less able to cope with such disasters and to adapt to climate change.

Another particularly important impact of climate change is increased susceptibility to health problems. A warmer climate could have adverse impacts on human health. Extremely hot weather would lead to increased heat stress, threatening especially old people and those suffering from cardiac diseases. Many infectious diseases would also be exacerbated by climate change such as: malaria, dengue fever, lymphatic filariasis, Japanese encephalitis, and arboviral diseases that are common in humid tropical regions like Vietnam. Climate change would facilitate the increased number and spread of insect vectors, leading to an increase in patients and death rates and greater pressure on health services. Taking the case of dengue fever, we will look more closely at the current and potential effects of climate change on that public health issue.

\subsubsection{The burden of dengue fever in the world and in Vietnam}

Dengue is regarded as the most important arboviral disease of humans in terms of morbidity and mortality (WHO, 1997). Dengue fever (DF) has been recognized by WHO as the fastest spreading tropical disease across all continents (WHO, 2008) (2.1). Bhatt et al. (2013) estimated that the global number of new infections per year (390 million, 95\% confidence interval: 284-528) is greatly under-reported: only 96 million cases (95\% confidence interval: 67-136) are recorded annually. 


\section{CLIMATE CHANGE AND DENGUE TRANSMISSION: EVIDENCE AND IMPLICATIONS FOR VIETNAM}

Dengue virus occurs in four serotypes: DENV-1, DENV-2, DENV-3, DENV-4; it belongs to the genus Flavivirus (family Flaviridae). Infection with dengue virus causes a wide range of clinical symptoms, ranging from a mild, undifferentiated fever, to classical dengue fever (DF) or dengue (also known as breakbone fever because of the pain it causes in joints), through to dengue hemorrhagic fever (DHF) and dengue shock syndrome (DSS). The clinical symptoms and resulting debilitation can last for weeks or months before recovery and return to full function.

The virus is transmitted by bites of female Aedes aegypti or Aedes albopictus mosquitoes in the inter-tropical regions of the world. It adversely impacts the health, social and economic status of these countries by causing a significant loss of life as well as debilitation, creating a major economic burden on society (Shepard et al., 2013). In the absence of an effective vaccine (although one is under development), mosquito control is the only available method of prevention, but that too is characterized by a lack of success and sustainability (Chadee et al., 2005; Halmar and Peter, 2011; McConnell and Gubler, 2003).

Patterns of dengue infection have depended to a great extent on the effectiveness of control efforts, together with socio-economic and ecological developments. Global warming may increase the number of people at risk of dengue infection by expanding both the area suitable for the mosquito vectors and the length of the dengue transmission season in temperate areas, whereas increased flooding may create stagnant bodies of water and perfect breeding grounds for the mosquitoes, exacerbating disease spread. Researchers in Australia and New Zealand calculated that climate change is projected to increase the range and risk of dengue, bringing 1.4 million Australians into newly risky areas suitable for the mosquito vector by 2050. Moreover, the number of months suitable for transmission may rise, increasing the costs of dengue management three to five times (McMichael et al., 2006).

Located in Southeast Asia, Vietnam is in the heart of this endemic area for dengue 


\section{CLIMATE CHANGE AND DENGUE TRANSMISSION: EVIDENCE AND IMPLICATIONS FOR VIETNAM}

and dengue hemorrhagic fever (DHF). It is recognized as a major cause of mortality and morbidity in Vietnam; for many years, it ranks amongst the top ten communicable diseases in terms of overall health burden (NIHE, 2009). In 1958, the first case of DHF was described in Vietnam (Mihov et al., 1959) and then, in 1963, the first reported outbreak occurred in Southern Vietnam, which resulted in 116 deaths (Ha and Huan, 2000). Major epidemics have occurred in 1969, 1983, 1987, 1998 and 2009. Based on the numbers of cases reported to World Health Organizations DengueNet (WHO, 2009), the 1987 dengue outbreak with 354,517 cases and 1,566 deaths, represented the single largest reported outbreak of dengue in the world. The estimated reported annual morbidity and mortality rates between 1979 and 2005 were 33-462 cases and 0.1-2.7 deaths, respectively, per 100,000 population per year. There has been an upward trend of dengue fever in all areas of Vietnam since the year 2004, with notable rises in 2009 and 2010. The total dengue cases in the whole country were 105,370 cases for 2009 and 128,710 for 2010, an increase of $22.15 \%$ compared to the previous year (Duoc et al., 2011). Although dengue transmission occurs in both rural and urban areas in Vietnam, $73 \%$ of Vietnams population live in rural areas and therefore the majority of dengue cases and deaths are from these areas (Tien et al., 2001).

Dengue transmission occurs throughout the year in Vietnam, with peak numbers of cases $(72 \%$ of total) reported between June and November. There are, however, regional differences in the climate in Vietnam and therefore seasonal transmission patterns can vary. For example, the Northern and Central Highland regions have a cool, dry winter from December to March each year, and dengue notifications are low during this time. Areas in Southern Vietnam have a warm stable climate throughout the year and dengue transmission can occur throughout the year, with peak transmission occurring between July and September (Ha and Ninh, 2000), which coincides with the rainy season in the South. In general, the majority of dengue cases have been reported from Central to Southern Vietnam, with yearly incidence rates in some 


\section{CLIMATE CHANGE AND DENGUE TRANSMISSION: EVIDENCE AND IMPLICATIONS FOR VIETNAM}

central coast communes of 2,418 cases per 100,000 population ( $2.4 \%$ of population with dengue) (Vu et al., 2005). From 1996-2007, the majority of dengue cases (77\%) and deaths (90\%) have been in Southern Vietnam. All four dengue virus serotypes have been found circulating in Vietnam, but the dominant serotype has varied over time. Between 1991 and 2007, DENV-1 and DENV-2 were the dominant serotypes circulating in Vietnam. DENV-3 emerged in the late 1990s causing the large outbreak in 1998, followed by a rise in the circulation of DENV-4 between 1999 and 2003. In recent years, DENV-1 has emerged as the predominant serotype in Vietnam (Duoc et al., 2011).

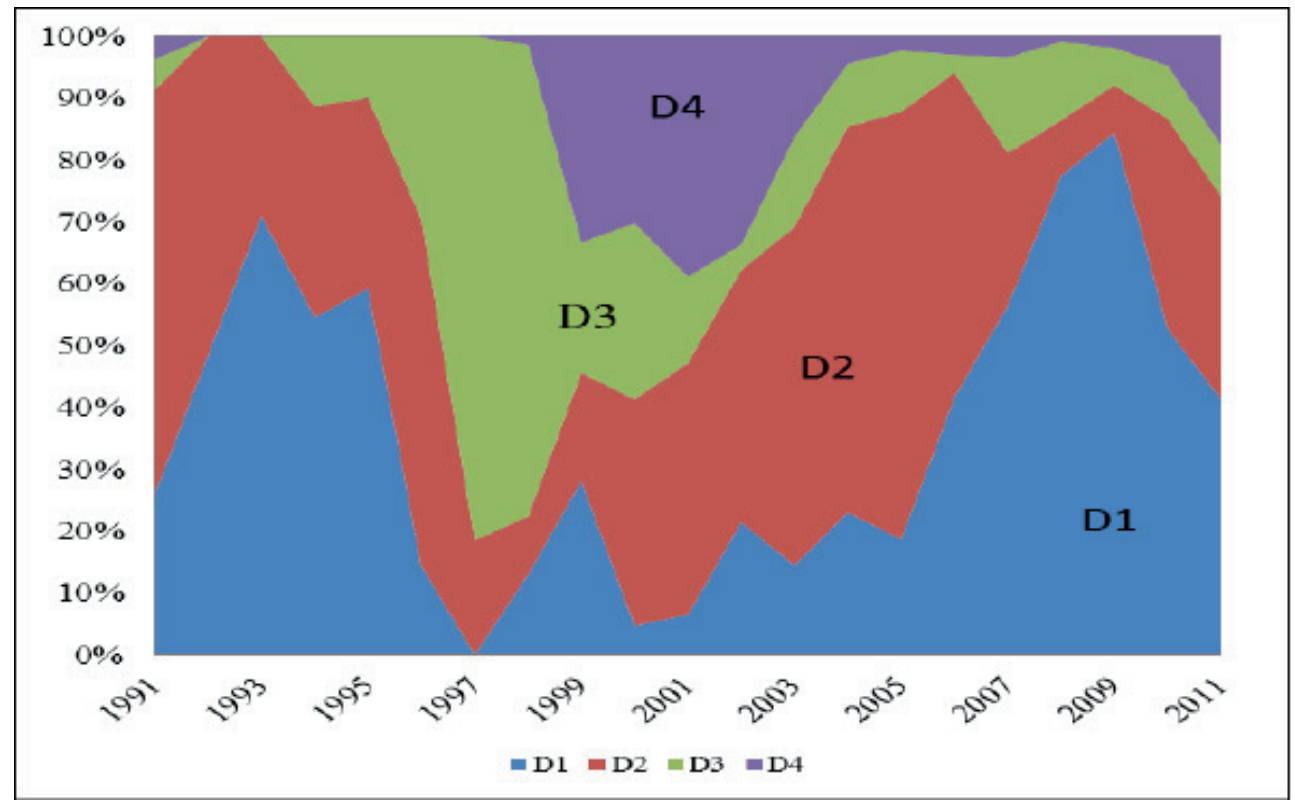

Figure 2.1: Distribution of dengue types in Vietnam from 1991 to 2011.

source: Vietnam National Institute of Hygiene and Epidemiology 


\section{CLIMATE CHANGE AND DENGUE TRANSMISSION: EVIDENCE AND IMPLICATIONS FOR VIETNAM}

\subsubsection{Dengue vector surveillance and control in Vietnam}

No vaccine or chemotherapy for dengue fever is available yet. Prevention and control of dengue therefore depend on vector control (larvicide treatment, insecticide sprays and elimination of breeding sites) and avoidance of bites. The national dengue control program in Vietnam recommends vector control by larvae elimination.

Vietnams National Dengue Control Program was first established in six provinces (Nam Dinh, Thanh Hoa, Khanh Hoa, Ho Chi Minh City, Minh Hai and Can Tho) in 1997 (Ha and Huan, 2000). The original program included active disease surveillance based on serological confirmation of suspected dengue cases, improved case management, emergency vector control incorporating ULV insecticide treatment around patients houses and to a radius of 25 metres to reduce the number of infected mosquitoes, and development of community based biological control programs which incorporated the larvivorous Mesocyclops. The program was expended in 2001 to 51 provinces, and is currently applied in all 64 provinces in Vietnam.

The selection of target communes as part of the National Dengue Control Program, including the type and level of control to be used, is made at the national level through the Ministry of Healths regional institutions, and activities are generally implemented by the district health centres with assistance from commune health staff (Knox et al., 2007). Currently, entomologic surveillance is conducted on a 1-3 monthly basis at communes identified as being at high risk of dengue transmission based on previous epidemic activity (Nguyen et al., 2011). Program staff undertakes routine sampling for adult mosquitoes at 50-100 randomly selected houses in each commune. Communes represent the lowest administrative unit in Vietnam. There are 9,069 communes throughout Vietnam, usually comprising 1,500 2,000 households within an area of approximately 20 square kilometers. During each survey, data from each house (number of each type of water container, capacity, fullness and the presence of Mesocyclops and other predators) are recorded, and adult mosquitoes and a subsample of immatures from each container are entered into a standardized spreadsheet that contains predefined calibration factors to convert the number of immatures obtained from quantitative net sampling to estimates of total immature abundance. These data are then collated by container type (e.g., large tanks, standard jars, waste containers) as a basis 


\section{CLIMATE CHANGE AND DENGUE TRANSMISSION: EVIDENCE AND IMPLICATIONS FOR VIETNAM}

for identification of key container types (Tun-Lin et al., 1995) and from that, prioritization of control activities, also for monitoring control effectiveness. As these data are aggregated at the commune level, they provide a broad assessment of the relative productivity of the various container types across the whole commune.

The vector control activities that form part of the National Dengue Control Program are generally community based, and involve a system of $25-50$ health volunteers per commune. Each volunteer is responsible for mobilizing community activities (Mesocyclops distribution to households, clean-up campaigns to remove discarded containers) and for conducting monthly entomology surveys at 50-100 households. Because these health volunteers are sourced from the local community, they are generally responsible for contiguous groups of households located in their own neighborhoods, hence the health volunteers are able to tailor the community based control activities to the local situation. However, these measures are usually only implemented after notification of severe cases (DHF and DSS) (WHO, 1997). This local policy is based on the assumption that such cases reflect locally increased vector densities with higher infection rates. It remains unclear to what extent this approach controls further transmission, because the majority of dengue virus infections ( $80 \%$ ) are mild/atypical or even asymptomatic (Burke et al., 1988). It is therefore likely that such measures are not adequate to prevent sustained dengue virus transmission in the community (Beckett et al., 2005; Chen and Wilson, 2004).

In fact, the geographical distribution of dengue infection has been expanding and transmission rates have increased over the last decades (Kroeger and Nathan, 2006). As mentioned above, increases in dengue incidence and severity are related to many factors including urbanization, increasing migration from rural to urban areas, mobility of people up and down the country, habitat pollution, floods, and climate factors such as temperature, rainfall, and humidity. However, the Government of Vietnam is currently still not considering how outbreaks of DF might be caused by climate change impacts. 
Chapter 3

Conceptual and

methodological framework 


\subsection{Climate change and vector-borne disease: a frame- work for analysis}

Many potential human health effects have been hypothesized to result either directly or indirectly from climate change, among which changes in the prevalence and spread of infectious diseases are frequently cited as potential results of climate change (Bouzid et al., 2013; Chan et al., 1999; Martens et al., 1995b; Reiter, 2001; Semenza and Menne, 2009). The direct effects of the anticipated changes in global and regional temperature, precipitation, humidity, and wind patterns resulting from anthropogenic climate change can be expected to have a direct impact on the vectors" reproduction, development rate and longevity. Thus, these factors would be associated with changes in vector density (Hunter, 2003; Martens, 2002).

Indirectly, climate change could also have an effect by influencing environmental factors such as vegetation and the availability of breeding sites. Otherwise, the influence which climate change is likely to exert on human populations may play an important role in the dynamics of disease transmission. For example, the dynamics of climate change (including sea-level rise in low-lying coastal areas) could strongly affect the migration of populations from areas in which vector-borne diseases are endemic into receptive areas (areas in which vector numbers and climate conditions are conductive for transmission) because of coastal rural impoverishment.

The influence of climatic conditions on vector-borne diseases such as malaria or dengue fever has been extensively studied. Climate change has already caused some changes in the prevalence and distribution of dengue fever and will continue to affect it (Bouzid et al., 2013; Gubler et al., 2001; Khasnis and Nettleman, 2005; Martens, 1998; Sutherst, 2004). Dengue prevalence is likely to be affected by climate change due to the fact that the mosquito vector is cold-blooded and therefore sensitive to changes in temperature, humidity or precipitation (Cuong et al., 2011; Hales et al., 2002; Khasnis and Nettleman, 2005; Martens et al., 1995a; Tanser et al., 2003). A study in Brazil showed that precipitation, temperature and relative humidity occurring three months previously were positively correlated with dengue, 


\section{CONCEPTUAL AND METHODOLOGICAL FRAMEWORK}

but precipitation and relative humidity had a negative relationship when occurring in the same month as dengue. The authors suggested that warm, humid conditions could promote mosquito development in the three months prior to increased dengue incidence, while heavy rainfall during the same month could wash out mosquito breeding habitats and inhibit dengue transmission (Lowe et al., 2008). Other studies have highlighted the relationship between climate and dengue transmission. An increase of temperature has been reported to be associated with dengue in Thailand, Indonesia, Singapore, Vietnam and Puerto Rico. Elevated humidity with high mosquito density increased the transmission rate of dengue infection in Southern Taiwan. Meanwhile, high rainfall has been linked to dengue fever in Indonesia, Trinidad, Venezuela, Barbados, Thailand and Vietnam. Temperature is linked to dengue incidence since temperature affects both the mosquitos longevity and biting rate, and the rate of viral reproduction within the mosquito (Gubler, 1998; Hales et al., 2002). Higher temperatures can shorten the extrinsic incubation period (viral development rate) of the dengue virus within the mosquito; conversely, very high temperatures may actually shorten mosquito survival (Patz et al., 1998). Meanwhile, rainfall increases the number of breeding places for mosquitoes, and high wind velocity could contribute to reduce mosquito density (Gharbi et al., 2011; Lu et al., 2009).

In addition to climatic factors, it is apparent now, with changes of other global drivers such as pollution, land use patterns, urbanization and human mobility, that dengue fever is now spreading outside the humid tropical and sub-tropical urban centers where it had been commonly found (Hay et al., 2002; Jacobs et al., 2005; Sutherst, 2004). It is therefore important to develop an integrated framework to identify the key plausible links and interactions that may influence the potential effects of climate change on vector borne disease, in particular on dengue fever. 


\subsection{Knowledge gap in analyzing dengue fever-climate change relationships}

Climate change would not affect the infectious diseases in isolation, but simultaneously and in conjunction with other ecological and demographic changes. This cause-effect relationship is described detail in MIASMA (Modeling framework for the health Impact ASsessment of Man-induced Atmospheric changes) which has been developed since 1997 (Janssen and Martens, 1997). This modeling approach has tried to cover as much as possible of the cause-effect relationship with respect to global atmospheric changes and human health. For example, to examine the potential risk of dengue fever posed by global climate change, Patz et al. (1998) applied global circulation models (GCM) toward projections and used a modified vectorial capacity equation to estimate the epidemic of dengue. They found that dengue incidence potential increased with a relatively small temperature rise, which indicated that lower mosquito infestation values could still maintain or spread dengue in a vulnerable population. Other studies have employed other statistical analytical methods to investigate this complex relationship. Gharbi et al. (2011) conducted a study in French West Indies, which fitted a seasonal autoregressive integrated moving average (SARIMA) model to data on dengue incidence and temperature, precipitation and relative humidity from 2000 to 2006. This model was also applied in Hus study to identify the impact of El Niño on dengue in Queensland, Australia from 1993 to 2005 (Hu et al., 2010). In both studies, it was found that including temperature significantly improved the ability of the model to forecast dengue cases. Earnest et al. (2012) and Pinto et al. (2011) applied a Poisson regression model to determine the relationship between meteorological variables and dengue in Singapore. The results of Earnest suggested a similar predictive ability for all the climatic variables, while Pinto reported that temperature was the best predictor. In Taiwan, Chen and Hsieh (2012) used Poisson regression with a GAM model to examine the relationship between dengue fever and precipitation. This GAM model could help to evaluate the multiple-lag effects of stratified precipitation levels on dengue disease. Descloux et al. (2012) used a multivariate non-linear model to develop an early warning system for dengue cases and climatic data. 


\section{CONCEPTUAL AND METHODOLOGICAL FRAMEWORK}

Their study again demonstrated a strong seasonality of dengue endemics and a relation with temperature, precipitation and relative humidity.

Although DF is now the most common vector born disease in Vietnam, relatively little research has been conducted on it. Thai et al. (2010) and Cuong et al. (2011) used a wavelet time series analysis, while Pham et al. (2011) used a Poisson regression model to investigate the associations between climate variables and dengue incidence in three provinces of Vietnam. There results revealed a significant association between dengue fever and climate change.

Many of the above methods have been successful in demonstrating a relationship between dengue and climate and in developing predictive models of dengue from this relationship. For example, Patz et al. (1998) have provided a good framework for future research on this relationship. However, the estimations should be updated based on better improved resolution with current GCM projections. Further, wavelet analysis is suitable for investigating the time series data from non-stationary systems and for inferring associations between such systems. Both Thai et al. (2010) and Cuong et al. (2011) used monthly dengue case data to show the coherence in 2-3 year periodic band. Nevertheless, to investigate the intra-annual patterns of seasonality, finer temporal resolution is needed for which data should be more aggregated.

There is still a debate as to whether dengue incidence increased because of climate change or resulting from socio-economic changes in combination with ecologic and demographic changes (Gubler, 2011). The question remains as to the role of climate change as a causal factor in the socio-economic and other changes that contribute to the spread of dengue in a country like Vietnam.

In summary, the recognition of the complex relationship between dengue fever and climate change demands the use of new approaches towards its analysis. Complexity theory helps understanding this relationship as the result of multiple elements interacting at different spatial and temporal scales. Analyzing the impacts of climate change on dengue fever requires the use of methodologies to deal with this complexity and uncertainty: Integrated Assessment (IA) provides the tools to deal with this need. 


\section{CONCEPTUAL AND METHODOLOGICAL FRAMEWORK}

\subsection{Role of Integrated Assessment}

The previous sections argued that the relationship between dengue fever and climate change is both complex and surrounded by uncertainties. Interdisciplinary and collaborative research in epidemiology, ecology, biostatistics and sociology is needed to address such complexity and uncertainty issues. Integrated assessments (IA) bring together and summarize information from diverse fields of study.

Integrated assessment is defined according to Rotmans (1998) as a structured process of dealing with complex issues, using knowledge from various scientific disciplines and/or stakeholders, such that integrated insights are made available to decision makers. In 2009, The Integrated Assessment Society (TIAS) has evolved this definition and made it more explicit: "the scientific 'meta-discipline' that integrates knowledge about a problem domain and makes it available for societal learning and decision making process". Although these definitions are slightly different, the basic idea behind IA is to combine, interpret and communicate knowledge from different scientific disciplines (Valkering et al., 2006) and from different stakeholders (Huynen and Martens, 2008). This kind of framework, therefore, is often used as a tool to help decision makers understand very complex environment problems.

In assessment of the cause-effect chain for climate change and dengue fever, integrated assessment refers to that activity that considers the direct and indirect impact of climate factors that drive the transmission of dengue fever. More specifically, the two defining characteristics of this integrated assessment are 1) that it brings together a broader set of areas, methods, styles of study, or degrees of certainty, than would typically characterize a study of the same issue within the bounds of a single research discipline; and 2) that it seeks to provide information not merely to advance understanding for its own purpose but also to inform decision makers. For example, the framework of integrated assessment in evaluating the potential impacts of climate change on infectious diseases includes not only pathogen transmission dynamics, but also contextual elements such as changing land use, and demographic forces such as population movement (Cox et al., 1999).

The methods developed and applied in the context of IA framework are commonly grouped together into two categories: analytical tools and participatory methods (Rotmans, 


\section{CONCEPTUAL AND METHODOLOGICAL FRAMEWORK}

1998; Valkering et al., 2006; Van Asselt et al., 2001). The diversity of analytical tools and participatory methods helps to perform assessments in an integrated way (Rotmans, 1998; van der Sluijs, 2001). Analytical methods (e.g. model analysis, scenario analysis and risk analysis) are adopted from the natural sciences and they are aimed at representing and structuring scientific knowledge in an integrated way. Participatory methods came from the social sciences; they are quite diverse, including focus group discussions, interviews, Delphi methods and policy discourses.

The aim of this research is to analyse the connections between climate change and dengue fever, including relevant social and ecological developments that may influence the relationship, and assess the possible impacts. In this thesis, the principles of IA have been incorporated by combining knowledge from different disciplines and research methods from the social and natural sciences. The way this has been done is presented in the next section.

\subsubsection{Research approach and methods}

An integrated assessment framework developed for this thesis is presented in Figure 3.1, which also illustrates how each study contributed to the whole. The objective of our framework is to identify the key plausible links and interactions that may influence the potential effect of climate change on human health and particularly on dengue fever. Other frameworks aim to model future health impacts of climate change at both global (or broad regional) level and local level (e.g. city/country), using global gridded projection data sets (Huynen and Martens, 2008; Martens, 1997). However, our approach was applied only at the local level in Hanoi City. We used real databases from local/national statistics stations to build up a statistical model based on the urban context of Hanoi. Thus, our application could show the potential of the proposed model for other similar contexts.

Both qualitative and quantitative approaches were applied in this thesis. We took a quantitative approach in addressing the direct and indirect impact of climate change on dengue fever. First, the direct impact of climate change on dengue fever was assessed with wavelet decomposition, a time-series statistical analysis (Chapter 4). This method allows to efficient deal with non-stationary data, which is the case for dengue in Hanoi where it is still 


\section{CONCEPTUAL AND METHODOLOGICAL FRAMEWORK}

an emerging disease. In Chapters 5, 6 and 7, we describe the results of assessing the indirect impact of climate change by investigating the potential influences of socio-ecological variation on the transmission of DF infection. A multivariable backward stepwise logistic regression model was established to describe the relationship between the dependent variable (get dengue infection or not) and a set of independent (predictor or explanatory) variables, such as age, education, occupation, having been in an epidemic area within past 6 months, urban or rural place of living, living in rented house or near open sewer, unhygienic environment, other family member had DF/DHF during past 6 months, presence of mosquitoes, presence of larvae in water containers, directly discharging sewage to ponds, and being flooded during the rainy season (Chapter 5). In Chapter 6, we applied a geographic information systems (GIS) technique to test for ecological links and map the risk of dengue fever with selected environment factors such as water bodies and vegetation. GIS are particularly well suited for the study of associations between location, environment and disease due to their spatial analysis and modelling capabilities (Gesler, 1986; Hu et al., 2004). Finally, the spatiotemporal distribution of DF infection and of epidemic foci were examined at the district level in Hanoi using space-time scan statistics (SaTScan), which is appropriate for detecting and evaluating the clusters of dengue cases in either a purely temporal, purely spatial or spacetime setting (Chapter 7). For a qualitative approach, we used participatory methods, such as scientist-stakeholder workshops and focus group discussions, to explore the perceptions of people in Hanoi about climate change and perceptions of the impact of climate change on health (Chapter 8). It is known that the quality of research can be improved by involving all relevant stakeholders, because they can contribute practical knowledge and experience, as well as range of different perspectives (Huynen et al., 2001; van Asselt Marjolein and RijkensKlomp, 2002). The following section outlines the main research methodologies applied in this study. In each of the following chapters of this thesis, methodological considerations will be presented in more detail. 


\section{CONCEPTUAL AND METHODOLOGICAL FRAMEWORK}

\subsubsection{Participatory methods}

The five participatory methods used in IA are focus groups, participatory modeling, scientist-stakeholder workshops, scenario analysis, and policy exercises. The transdisciplinary approach taken in this research project has been implemented through focus groups, stakeholder workshops, and in-depth interviews with policy makers. These methods help in different ways to identify knowledge about and perspectives on the complex issue of climate change and human health.

\section{Focus groups}

Focus groups consist of a limited number of stakeholders who join structured, moderated discussions designed to elicit preferences, opinions and viewpoints. Local political leaders, district health staffs and community members have knowledge and opinions about the current and future implication of climate change and health. They were invited to two stakeholder workshops held in Hanoi. A research progress report was presented at each workshop and participants were invited to give suggestions for improvement. A report providing details on the methodology, process and outcomes was prepared for each workshop. Each of the reports lists the stakeholders that participated during the workshops. To maintain the confidentiality of the data, specific ideas or results of the workshops were not attributed to individual participants. An overview of the workshops and the outcomes is presented in Chapter 4 .

Stakeholder workshops played an important role in the first part of this research; they contributed to the identification of knowledge about how recent climate change has affected Hanoi and may have led to an increase in health problems for its inhabitants. These findings laid the base for the following chapters to look more deeply into the real problems of climate change and health focusing on the case of dengue fever.

\section{In-depth interviews}

The in-depth interview is a qualitative research method "that involves conducting intensive individual interviews with a small number of respondents to explore their perspectives on a particular idea, program, or situation" (Boyce and Neale, 2006). It is characterized by the length, depth and structure of the interview and seeks to obtain more deep information 


\section{CONCEPTUAL AND METHODOLOGICAL FRAMEWORK}

then a questionnaire-based interview could do (Silverman, 2000). In order to obtain insights from key stakeholders not participating in the focus groups, in-depth interviews were conducted with them which were unstructured and often used a flexible approach. During the interviews, together with a semi-structured interview guideline, several open questions were asked reflecting the issues discussed during the workshops (Lamers et al., 2012). The interview guideline is included in the appendix of this thesis. Interviews were audio-recorded, transcribed, and sent back to the interviewees for revisions and approval.

In-depth interviews were done with the political leaders of two representative slum and non-slum districts in Hanoi, to find out about their perceptions on climate change in general and specifically on its implications for human health. Furthermore, their responses contributed to our understanding of the current situation of climate change and vulnerability of people living in Hanoi to climate change (for details see Chapter 4).

\subsubsection{Analysis of quantitative data}

Quantitative data analysis is helpful in evaluation because it provides quantifiable and easy to understand results. Quantitative data can be analyzed in a variety of different ways. In this thesis, we have applied several tools for quantitative analysis that are mentioned above: wavelet analysis, logistic regression, spatio-temporal analysis with GIS, and SatScan analysis.

- Wavelet analysis is a common tool, which helps to analyze localized variations of power within a time series. Both the dominant modes of variability and how those modes vary in time are easier to determine by decomposing a time-frequency space (Torrence and Compo, 1998). Wavelet analysis has been applied successfull in ecology and epidemiology, and it appears particularly attractive given the non-stationary nature of both ecological and environmental time series and the relationships between these series.

- Logistic regression is important for modeling dichotomous dependent variables and is often used in epidemiological studies to state an individuals risk for developing a disease (Hosmer and Lemeshow, 2000).

- GIS is an integrated set of computer hardware and software tools designed to capture, store, 


\section{CONCEPTUAL AND METHODOLOGICAL FRAMEWORK}

retrieve, and display spatially-referenced data (Bailey and Gatrell, 1995). The applications of GIS technology superimpose the temporal and spatial distributions of the socio-ecological determinants of endemicity of DF (eg. climate, landscape ecology, reservoir and vector populations, and human presence and activity).

- Scan statistics are used to analyze spatial, temporal, and space-time data using spatial, temporal, or space-time scan statistics. Its function is to detect and evaluate the clusters of cases in either a purely temporal, purely spatial or space-time setting (Kulldorff, 1997).

For these analyses, different types of data are gathered from different sources: surveillance health system, national meteorology system and interviews with patients using structured questionnaires.

- Daily data on DF cases between January 2003 and December 2011 in Hanoi were obtained from the Hanoi Center for Preventive Health. Data included the onset date and place for the notified cases of DF infection, plus the age, sex and occupation of the patients and the laboratory test results. The criteria for notification of DF disease are based on Ministry of Healths 2006 guidelines on surveillance, diagnosis and treatment of dengue (MOH, 2006).

- Daily weather data for Hanoi from 2003 to 2011 were provided by the National Centre for Hydrometeorological Forecasting. They include the records of mean, maximum, and minimum temperatures $\left(\mathrm{T}\right.$, in ${ }^{\circ} \mathrm{C}$ ), rainfall (in $\mathrm{mm}$ ) and relative humidity (in \%).

- The population data for each commune/district in Hanoi for the years 2003 and 2011 were obtained directly from the decennial National Population and Housing Census, conducted by the General Statistics Office of Vietnam (GSO, 2008)

- Data collection from a structured questionnaire was conducted with two groups of patients with and without notification of DF. The majority of the questions were multiple choice with a few open questions, and covered all information on demographic characteristics of the patients.

In summary, a large scale integrated assessment approach was used in this study. These methods can improve our understanding of the biological/ecological mechanisms of disease outbreaks and assist in assessing the spatio-temporal relationships between risk factors and disease transmission (Hu et al., 2004). The key findings are presented in each of the following chapters. 


\section{CONCEPTUAL AND METHODOLOGICAL FRAMEWORK}

\subsubsection{Study limitations}

The correlation between climate change and population health is complex and still poorly understood. Our study aimed to demonstrate this correlation in the case of dengue fever. However, it could not represent all of the issues in this complicated association.

The research described in this thesis did have a few methodological limitations. The studied issues were complicated and difficult to measure. From Chapter 4 to Chapter 7, we report on application of integrated assessment models that include several approaches. The major limitation of these approaches was control for the uncertainties,. We only studied a part of the ecological and social factors (climatic variables, vegetation, mosquito density, housing conditions and personal health behavior) while other factors (such as population immunity, mosquito control measures, and human population movement) were not included because data were unavailable. These major limitations of the study need to be considered in future research on DF/DHF disease.

Another restriction of applying models is that the precision of a forecasting model is largely influenced by the quality of the registered data it is based on. In Hanoi, there is a network of commune health stations located in each village all over the city. Each health station is in charge of dengue surveillance and reports the surveillance data to the district which transmits it monthly to the Hanoi Preventive Medicine Center. However, the data based on this surveillance system might be underestimating real incidence. To our knowledge, a number of dengue patients were treated in private health services that are not included in state system records. A study on the diagnosis of acute undifferentiated fever in Vietnam showed that acute dengue was found in $34 \%$ cases, which suggests the possibility of under-reporting of dengue in commune health stations (Pham et al., 2011). WHO classification schemes introduced in 1997 and 2009 had high sensitivity but lacked specificity (Chaterji et al., 2011). As in many dengue-epidemic settings, the dengue surveillance system in Vietnam relies on passive reporting of clinically-diagnosed dengue in hospitalized patients. Less severe infections will probably not reach hospitals. Therefore, in reality the number of dengue infections will be much higher than the reported number (Cuong et al., 2011). The assumption that the numbers of clinically reported cases systematically reflect the numbers of unreported 


\section{CONCEPTUAL AND METHODOLOGICAL FRAMEWORK}

cases in the same area is difficult to validate. However, in our study, the analytic units were monthly/weekly incidence, monthly/weekly climate, monthly/weekly high tide time-series data. The impact of other social and ecological factors on the transmission of DF infection may be minimal, because they were unlikely to change dramatically within such a short time frame.

Also, in Chapter 8, we report on people's perceptions about their health and possible changes related to climate, but we could not check against real data on health changes. In addition, there were limitations using participatory workshops, especially the difficulty of involving all stakeholders, of getting their continued commitment around the table, to ensure that the ideas and opinions of all participants could be recorded. Figure 3.1 presented the research assessment framework of the thesis.

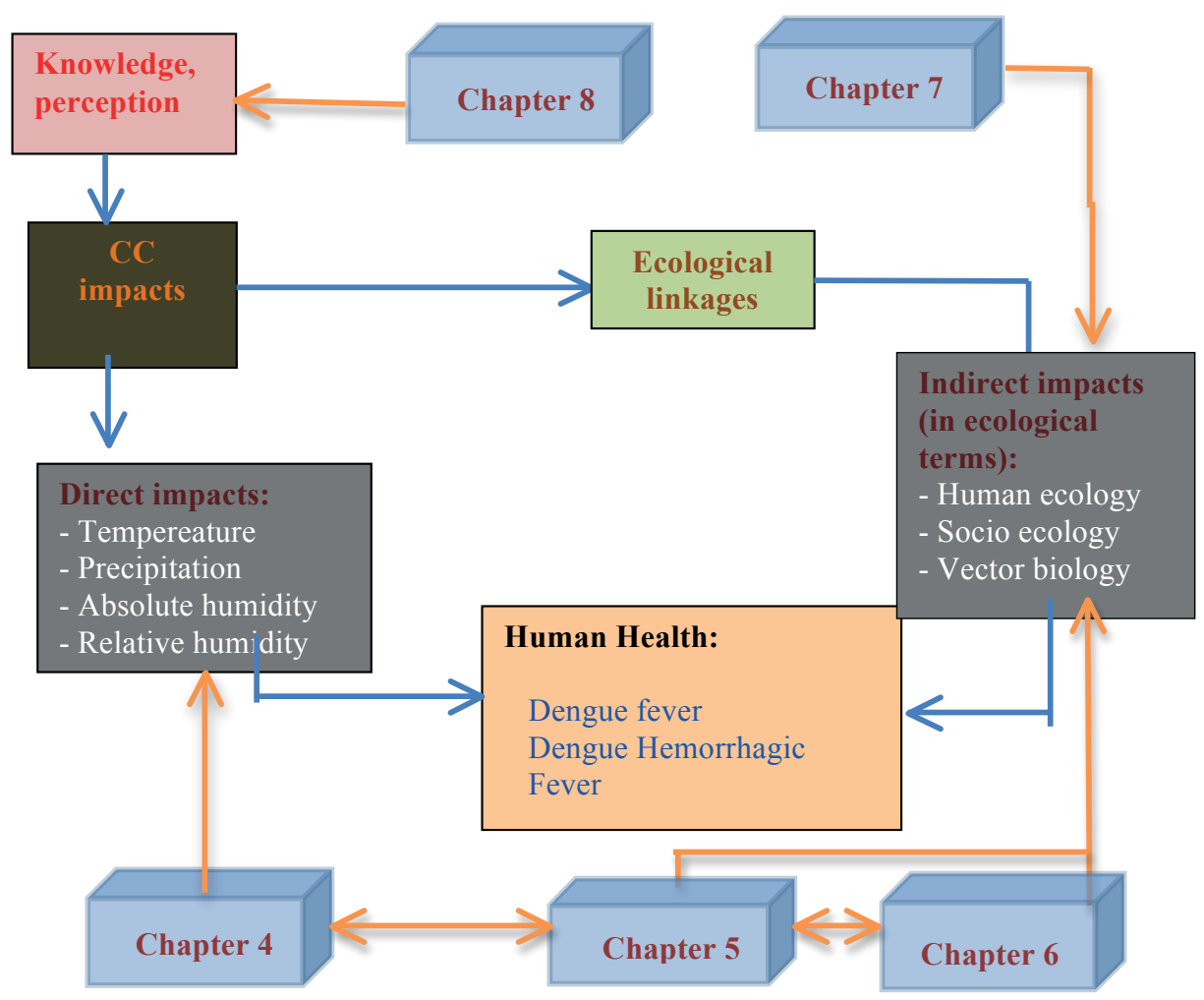

Figure 3.1: Research assessment framework 


\section{Chapter 4}

\section{Climatic-driven seasonality of}

\section{emerging dengue fever in}

\section{Hanoi, Vietnam}

This chapter has been published as: Toan DTT, Martens P, Hoat LN, Wright P, Choisy $M$ (2014). Climatic-driven seasonality of emerging dengue fever in Hanoi, Vietnam. BMC Public Health 2014, 14: 1078. 


\section{CLIMATIC-DRIVEN SEASONALITY OF EMERGING DENGUE FEVER IN HANOI, VIETNAM}

\subsection{Introduction}

Dengue Fever (DF) has recently been recognized by WHO as the fastest spreading tropical disease across all continents. Bhatt et al. (2013) estimated that the global number of new infections per year (390 millions, 95\% confidence interval: 284-528) is largely underestimated: only 96 millions (95\% confidence interval: 67-136) being declared yearly. DF is one of the many symptoms (ranging from mild fever to hemorrhagic fever and shock syndrome) caused by one of the four serotypes of dengue virus (Flaviridae family). Even though recovery from dengue confers life-long immunity against the infecting serotype, immunological interactions between the different serotypes are not fully understood. The virus is transmitted by bites of female Aedes aegypti or albopictus mosquitoes in the inter-tropical regions of the world. In absence of vaccine (under development), mosquito control is the only available method of prophylaxy. In Vietnam, dengue is recognized as a major cause of mortality and morbidity and ranks amongst the top ten communicable diseases in terms of overall health burden (Tien et al., 1999). All four dengue virus serotypes have been found circulating in Vietnam with the dominant one varying over time. Reports from the National Institute of Hygiene and Epidemiology show that DENV-1 and DENV-2 have been the predominant circulating viruses almost every year. DENV-3 emerged in the late 1990s and was responsible for the large outbreak of 1998, whereas DENV-4 was also detected between 1999 and 2003 (NIHE, 2009). Dengue transmission occurs throughout the year in Vietnam, with peaks in the numbers of cases ( $72 \%$ of total cases) reported between June and November (Ha and Ninh, 2000). There are regional variations in the seasonality of dengue epidemiology in Vietnam. In the Northern and Central Highland regions, dengue notifications are low during the winter time from December to March, while in southern regions, dengue transmission occurs throughout the year, even if it sharply increases during the rainy season from July to September. Given that dengue is a vector-born disease and that vector population dynamics are strongly dependent on climatic factors, the diversity of climates in Vietnam may explain the observed diversity of dengue epidemiological dynamics. Hanoi, the capital of Vietnam, is located in the North of the country and known as a low transmission setting of DF (Cuong et al., 2011). Hanoi experiences annual seasonal dengue outbreaks with the pinnacle of epi- 


\section{CLIMATIC-DRIVEN SEASONALITY OF EMERGING DENGUE FEVER IN HANOI, VIETNAM}

demics usually falling in September/October and ending in November/December. Over the last decade, the number of DF cases has been increasing from year to year, reaching a peak in 2009. According to the Ministry of Healths statistics, the outbreak in Hanoi in 2009 is the most important outbreak of the last decade, with 384 notified cases per 100,000 individuals. Interestingly, 2009 was also the year El Niño increased actively (Minh An and Joacim, 2014; MOH, 2010). There are only few studies published on dengue epidemiology in Hanoi that are based on the public health surveillance data routinely collected through the Ministry of Healths notifiable diseases surveillance program. Toan et al. (2013) show that there are spatio-temporal clusters of DF limited to a radius of $1,000 \mathrm{~m}$ and a duration of 29 days. This study also demonstrates that most of the DF cases occur between June and November, during which the rainfall and temperatures are highest. Cuong et al. (2011) use wavelet analysis to relate dengue incidence to climatic variables and suggest that all the tested local climatic variables (total rainfall; mean wind velocity; mean, maximum and minimum temperatures; relative humidity) are significantly associated with dengue incidence around the annual periodicity: on average, dengue incidence follows the seasonal peak of rainfall and mean temperature with a lag of 1 to 2 months. Other studies have been carried out on the correlation between climate and DF in other parts of Vietnam as well as in other parts of the world, using a wide spectrum of mathematical and statistical modeling methods (Chowell et al., 2008; Depradine and Lovell, 2004; Descloux et al., 2012; Gharbi et al., 2011; Hales et al., 2002; Hsieh and Chen, 2009; Hsieh et al., 2013; Hu et al., 2010; Johansson et al., 2009; Lu et al., 2009; Nagao et al., 2003; Pham et al., 2011; Pinto et al., 2011; Promprou et al., 2005a,b; Schreiber, 2001; Wu et al., 2007). In Vietnam, most of the studies have been carried out in the south and the center of the country, and showed significant associations between climatic variables and dengue incidence. A wavelet analysis of monthly dengue cases from the province of Binh Thuan has shown a non-stationary relationship between El Niño Southern Oscillation indice and dengue incidence in the 2-3 year periodic band (Thai et al., 2010). Meanwhile, a correlation study carried out on monthly dengue cases from the province of Daklak has found the risk of dengue to be associated with high temperature, high relative humidity and rainfall, but inversely associated with duration of sunshine (Pham et al., 2011). Findings from other studies in many other parts of the world also show cli- 


\section{CLIMATIC-DRIVEN SEASONALITY OF EMERGING DENGUE FEVER IN HANOI, VIETNAM}

matic variables to have an effect on dengue transmission. Studies in Thailand (Nagao et al., 2003), Barbados (Depradine and Lovell, 2004), Taiwan (Hsieh and Chen, 2009), Guangzhou, China (Lu et al., 2009), the French West Indies (Gharbi et al., 2011) and in Colombia (Eastin et al., 2014) showed a positive correlation between dengue incidence and minimum and maximum temperatures, precipitation and relative and absolute humidities. However, depending on the approach of analysis and the areas, these correlations were more or less strong. In Barbados, the strongest correlation was found at a lag of 6, 12 and 16 weeks for vapour pressure and minimum and maximum temperatures respectively, whereas in Taiwan the highest correlations were found with maximum temperature at a lag of 5 weeks and with total precipitation at a lag of 7 weeks. In Colombia, Eastin et al. (2014)'s results suggest that DF cases increase 2 to 5 weeks after the daily temperature range remains for an extended period within the temperature range optimal for vector survival and disease transmission. Nagao et al. (2003) in Thailand and Yi et al. (2003) in Guangdong, China, demonstrated that the distributions of Aedes species and dengue cases were positively associated with high absolute humidity, which itself increases with high temperature and rainfall. In San Juan, Puerto Rico, Schreiber (2001) used a water budgeting technique and showed that high levels of dengue are associated with reduced actual evapotranspiration, minimum temperature and with high levels of precipitation. In Taiwan, using autoregressive integrated moving average models, Wu et al. (2007), found a negative association of dengue incidence with temperature and relative humidity. Finally, in the city of Noumea (New Caledonia), Descloux et al. (2012) recently documented a high seasonality of dengue incidence, with an epidemic peak (March-April) lagging the warmest temperature by 1 to 2 months and in phase with maximum precipitations, relative humidity and entomological indices. In the present study, we consider vapor pressure and relative humidities, temperature and rainfall in order to identify which of these variables are most critical for the onset of dengue epidemics. Compared to Cuong et al. (2011), who also investigated the links between dengue and climatic variables in Hanoi, Vietnam, we here consider vapor pressure in addition to relative humidity. Vapor pressure is a measure of absolute humidity and this climatic variable is often neglected in the studies investigating the links between climate and disease transmission, even though it has been proved to play a role more important than relative humidity for the transmission of 


\section{CLIMATIC-DRIVEN SEASONALITY OF EMERGING DENGUE FEVER IN HANOI, VIETNAM}

some diseases such as influenza Shaman and Kohn (2009). A second difference with Cuong et al. (2011) is that we here work on weekly incidence aggregates instead of monthly aggregates. With this finer temporal resolution we aim at investigating intra-annual patterns of seasonality. We first quantify the synchrony between weekly dengue incidence and the four climatic variables between 2002 and 2009. We then estimate the values of reproductive ratio of dengue fever through time and characterize the trend and seasonality of reproductive ratio. We finally discuss the results in light of immunological and entomological factors specific to dengue epidemiology in Hanoi, Vietnam.

\subsection{Materials and Methods}

\subsubsection{Study setting}

Hanoi $\left(1,760 \mathrm{~km}^{2}\right)$ is located in the Red River Delta, in the centre of North Vietnam $\left(21^{\circ} 2 \mathrm{~N}, 105^{\circ} 51 \mathrm{E}\right)$. The city experiences the typical climate of northern Vietnam, where summers are hot and humid, and winters are, by national standards, relatively cold and dry.

\subsubsection{Data collection}

The data used in this study have been previously published by Cuong et al. (2011) and Toan et al. (2013). Clinical suspected cases of DF in old Hanoi (14 districts of Hanoi before merging with Ha Tay in 2008) are reported to the surveillance system of Hanoi Center for Preventive Health. The criteria for notification of DF disease are based on the guidelines of the Ministry of Health (2006) on surveillance, diagnosis and treatment of dengue, in which suspected dengue cases are based on acute febrile illness $\left(38^{\circ} \mathrm{C}\right)$ of 2-7 days duration with at least two of the following non-specific manifestations of dengue fever: headache, retro-orbital pain, myalgia, arthralgia, rash, hemorrhagic manifestations, and leucopenia (MOH, 2006). The data analyzed here include all reported cases from January 2002 to December 2009, aggregated by week. Daily weather data for Hanoi from 2002 to 2009 were provided by the National Centre for Hydrometeorological Forecasting. They include the records of mean, maximum, and minimum temperatures $\left(\mathrm{T}\right.$, in ${ }^{\circ} \mathrm{C}$ ), rainfall (in $\mathrm{mm}$ ) and relative humidity 


\section{CLIMATIC-DRIVEN SEASONALITY OF EMERGING DENGUE FEVER IN HANOI, VIETNAM}

(in \%).

\subsubsection{Vapor pressure}

We used vapor pressure (VP, in mb) as a proxy of absolute humidity. VP was calculated from relative humidity $(\mathrm{RH}$, in \%) and temperature $\mathrm{T}$ using the ClausiusClapeyron formula (Shaman and Kohn, 2009; Wallace and Hobbs, 2006):

$$
V P=V P_{0} * \exp \left[\left(\frac{L}{R_{V}}-T_{0}\right)\left(\frac{1}{T_{0}}-\frac{1}{T+T_{0}}\right)\right] \frac{R H}{100}
$$

where $\mathrm{L}=2,257 \mathrm{~J} / \mathrm{g}$ is the latent heat of evaporation for water, $R_{V}=416.5 \mathrm{~J} /(\mathrm{kg} \mathrm{K})$ is the gas constant for water vapor, $\mathrm{T}_{0}=273.15 \mathrm{~K}$ and $\mathrm{VP}_{0}=6.11 \mathrm{mb}$ is the vapor pressure at which water would change phase between vapor and liquid if the temperature was $T_{0}$. Note that in the above formula temperature $\mathrm{T}$ is expressed in ${ }^{\circ} \mathrm{C}$ instead of $\mathrm{K}$ as in Shaman and Kohn (2009). Climatic variables were aggregated by week using sums for rainfall and mean values for all the other variables.

\subsubsection{Wavelet analysis}

Epidemiological data can be substantially non-stationary (Cazelles et al., 2005; Cazelles and Hales, 2006; Simoes et al., 2013) as is the case for dengue in Hanoi where it is emerging (see in particular the increase in mean and amplitude over time on figure 4.1B). Here we performed wavelet decomposition, a time-series statistical analysis allowing to efficiently deal with non-stationary data. Specifically, we used the Morlet wavelet

(Cazelles et al., 2007), classically used in ecology with a non-dimensional frequency $\omega_{0}=$ 6. An advantage of using the Morlet wavelet is that it is a complex wavelet, allowing to quantify the phase and thus calculate time lags between different time series. Coherence based on wavelets allows to perform similar analysis as cross-correlation but for potentially non-stationary signals. Wavelet coherences were calculated to examine the association between two time series, both in time and frequency. Coherence spectra allow to investigate whether different periodic modes of two time series tend to oscillate simultaneously and, if yes, to identify the periodicity around which this association takes place. Significance levels 


\section{CLIMATIC-DRIVEN SEASONALITY OF EMERGING DENGUE FEVER IN HANOI, VIETNAM}

were calculated by a Chi-square test assuming that the wavelet coefficients are normally distributed as described in Torrence and Compo (1998). The detailed theory for wavelet analysis has been described elsewhere (Cazelles et al., 2007). Before wavelet decomposition, time series were square-root transformed in order to mitigate the weights of high values. They were also zero-padded to the next power of 2 of their length (i.e. 512), in order to minimize edge effects (Torrence and Compo, 1998).

\subsubsection{Reproductive ratio}

The reproductive ratio $R$ is the expected number of infections caused by one infected individual. It is maximal at the start of an epidemic when the host population is fully susceptible and then decreases over the course of the epidemics. It reaches the equilibrium value of 1 at epidemic peak and decreases below 1 after it. The initial and maximum value of the reproductive ratio is called the basic reproductive ratio $R_{0}$ and is the classical epidemiological statistics as its value relative to 1 informs about the potential for an epidemic to occur. We followed Soper (1929) as reported in Keeling and Rohani (2008) and approximated the reproductive ratio $\mathrm{R}$ by:

$$
R \approx\left(\frac{C_{t+1}}{C_{t}}\right)^{\alpha}
$$

where $\mathrm{C}_{t+1}$ and $\mathrm{C}_{t}$ are the numbers of cases at times $\mathrm{t}+1$ and $\mathrm{t}$ respectively and is a parameter that expresses the infection generation length (i.e. the sum of the infectious and latent periods) in the same units as the data time steps (here 1 week). The infection generation length for dengue being approximately 2 weeks (Andraud et al., 2012), we set this parameter to the value of 2. The other part of Soper (1929)'s formula expresses the reproductive ratio as a function of the number of susceptibles in the population:

$$
R \approx k \frac{X_{t+1}}{X^{*}}
$$

where $\mathrm{X}^{*}$ and $\mathrm{X}_{t+1}$ are the numbers of susceptibles before the epidemics and at time $\mathrm{t}+1$, respectively and $\mathrm{k}$ is a parameter reflecting some potential external forcings (such as climatic ones). This latter part of the Soper (1929)'s equation shows that variations in the 


\section{CLIMATIC-DRIVEN SEASONALITY OF EMERGING DENGUE FEVER IN HANOI, VIETNAM}

reproductive ratio are due either to variations in the number of susceptibles in the population, or to some external forcings directly affecting the transmissibility of the disease (in our case climatic factors acting on the vector population dynamics and density). Given that dengue is emerging in Hanoi and that the basic reproductive ratio of dengue is generally low (Andraud et al., 2012), we expect the depletion of susceptible in the population to be very slow. We expect it to be even slower given the fact that dengue can actually be caused by four different serotypes with no permanent cross-immunity between them. Hence, among the two abovecited factors that can affect the seasonality of the reproductive ratio (susceptible depletion and external forcing), we assume that susceptible depletion is negligible before any external forcing such as climatic drivers on the mosquito population dynamics. Time series of the calculated seasonal factor $\mathrm{k}$ were smoothed by lowess regression with smoothing factors equal to 0.05 and 0.90 in order to reveal its seasonality and its long-term trend respectively. Confidence intervals were calculated by assuming normal distribution of errors. Given the uncertainty on the infection generation length and the assumption made on the number of susceptibles, estimates of the reproductive ratio will be treated with caution: only their trend and seasonality will be interpreted, not their absolute values, on which we will have limited confidence. All analyses were conducted in R (RCoreTeam, 2012) and wavelet analyses were performed using the biwavelet R package (Gouhier and Grinsted, 2013).

\subsection{Results}

\subsubsection{Dengue incidence and its reproductive ratio in Hanoi}

From 2002 to 2009, 23,195 DF cases were reported in Hanoi with the average annual incidence rate of $69.22 / 100,000$. Overall, the incidence of DF increased over the 8 years of the study, with a sharp increase during the period 2005-2009. This period of increasing incidence is visible on the wavelet spectrum of figure 4.3.1C. The highest peak of 3,697 cases was recorded in September 2009. Over the 5 years from 2005 to 2009, annual (1 year) and sub-annual (6 months) periodicities were significant. These two periodicities correspond to a slow increase of DF incidence from the beginning of the year to weeks 22-24 (June), followed 


\section{CLIMATIC-DRIVEN SEASONALITY OF EMERGING DENGUE FEVER IN HANOI, VIETNAM}

by a rapid increase of incidence until weeks 44-46 (November) which ends by a sharp decrease in incidence at the end of the rainy season. Figure 4.3.1A shows a long-term increase in the reproductive ratio (orange curve) as well as a non-stationary sub-annual periodicity with two peaks of this reproductive ratio per year (blue curve). These peaks are of roughly equal magnitude from 2005 to 2008, the second peak is substantially higher than the first one in 2004 and 2009, and the number of cases are too low before 2004 for any clear pattern to be visible (large confidence intervals on figure 4.3.1B).

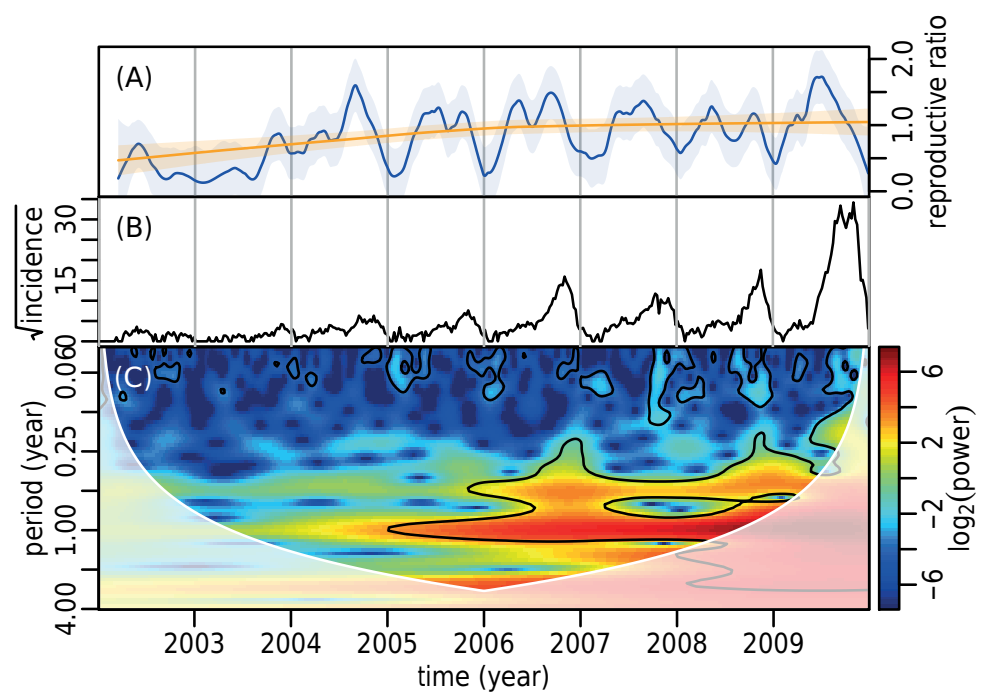

Figure 4.1: The reproductive ratio, time series and wavelet power spectrum of DF in Hanoi (14 districts) from 2002 to 2009.

(A) The reproductive ratio was estimated from equation 2 (see text) and smoothed by lowest regressions with smoothing factors equal to 0.05 (blue) and 0.90 (red). The shaded areas around the lines represent the $95 \%$ confidence intervals calculated assuming a normal distribution of errors. (B) Time series of the square-root transformed weekly DF incidence. (C) Wavelet power spectrum of the square-root transformed weekly DF incidence. The black contour lines show the regions of power significant at the alpha-risk of 0.05 . The paled region of the spectrum delineates the cone of influence due to the zero-padding of the time series. The power increases from dark blue to dark red 


\section{CLIMATIC-DRIVEN SEASONALITY OF EMERGING DENGUE FEVER IN HANOI, VIETNAM}

\subsubsection{Meteorological variables in Hanoi}

The wavelet power spectra of temperature, precipitation, and humidities (relative humidity and vapor pressure) in Hanoi during the study period are shown in Figure 4.2. Temperature, precipitation and vapor pressure in Hanoi show significant annual periodicities that are constant through time, whereas relative humidity shows both annual and sub-annual periodicities, as observed on the DF incidence time series (Figure 4.3.1B).

The cross-correlation coefficients among four climatic variables in Hanoi are presented in Figure 4.3. It shows that mean temperature, rainfall and vapor pressure are much correlated and in phase, whereas there is a significant lag between these three variables and relative humidity.

Over the 8 years of the study, the average temperature in Hanoi was found to be lowest in January and February $\left(16.07 \pm 1.65^{\circ} \mathrm{C}\right.$ standard deviation) and highest in June and July $\left(29.80 \pm 0.47^{\circ} \mathrm{C}\right)$. Over the studied period, the coolest temperature was observed in week 5 (February) of 2008 and the warmest was observed in week 27 (July) of $2009\left(10.11^{\circ} \mathrm{C}\right.$ and $32.84^{\circ} \mathrm{C}$ respectively). July to September was the time of the year receiving the majority of the annual rainfall (271.9 $\pm 111.9 \mathrm{~mm}$ in average over one week). Week 44 (November) in 2008 recorded a rainfall extreme of $576.6 \mathrm{~mm}$ brought by the typhoon Maysak (Center for Excellence in Disaster Management and Humanitarian Assistance, 2008). The relative humidity in Hanoi is quite high (78.62 $44.28 \%$ ), and is usually higher in February and March (cool but very rainy: $84.12 \pm 2.56$ ), and August and September (drier but very hot: 79.81 \pm 3.54 ), than the rest of the year $(76.80 \pm 3.99)$.

\subsubsection{Coherences between meteorological variables and DF in- cidence in Hanoi}

Results of wavelet coherences between DF incidence and climate variables are shown in Figure 4.4. Significant coherences were observed between DF incidence and temperature, precipitation and humidity for the annual periodicity from approximately 2005 to 2009 (Figure 4.4A-D). Moreover a weaker, but still significant association between relative humidity and DF incidence was also seen for the sub-annual periodicity from 2006 to 2009 (Figure 


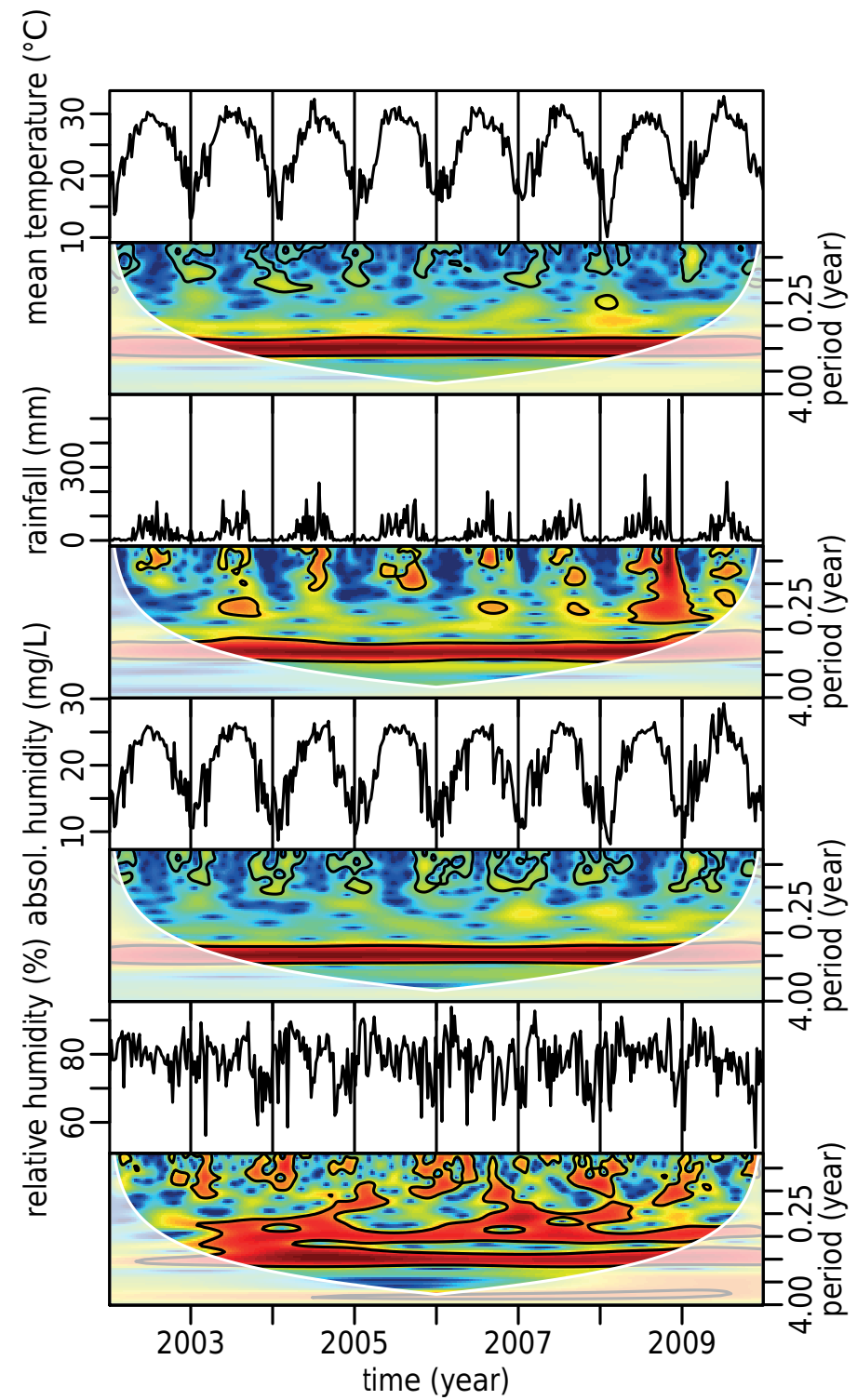

Figure 4.2: Time series and wavelet power spectra of mean temperature, cumulative rainfall and mean absolute and relative humidities in Hanoi, from 2002 to 2009.

The black contour lines show the regions of power significant at the alpha-risk of 0.05 . The paled region of the spectrum delineates the cone of influence due to the zero-padding of the time series. The power increases from dark blue to dark red. 


\section{CLIMATIC-DRIVEN SEASONALITY OF EMERGING DENGUE FEVER IN HANOI, VIETNAM}

\section{mean temperature}

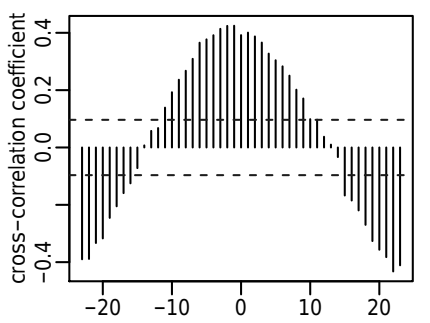

rainfall
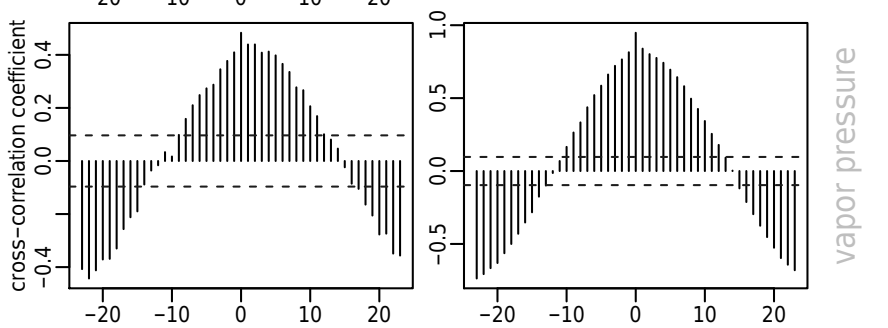

vapor pressure
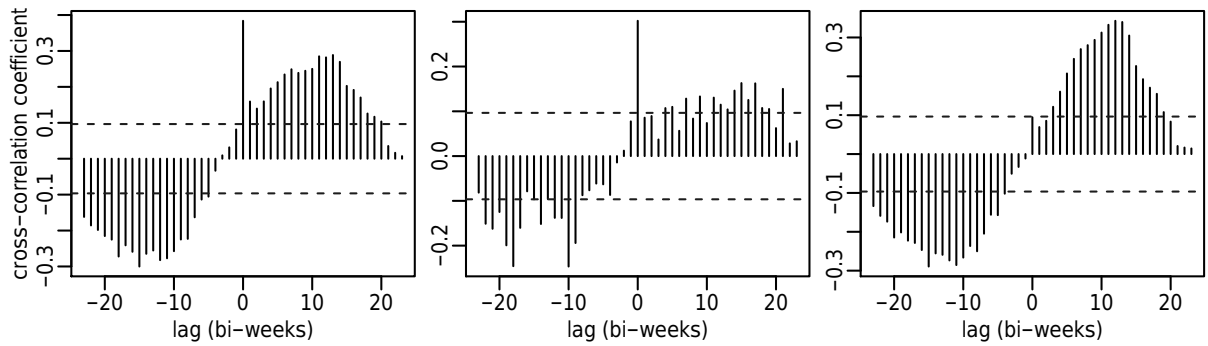

Figure 4.3: Cross-correlation between the 4 climatic variables: mean temperature, rainfall, vapor pressure and relative humidity

Horizontal blue dotted lines materialize the significativity thresholds at $\mathrm{p}=0.05$ 


\section{CLIMATIC-DRIVEN SEASONALITY OF EMERGING DENGUE FEVER IN HANOI, VIETNAM}

4.4D). In analyzing the phase difference at the annual cycle between DF incidence and the climatic variables, we found that dengue incidence was consistently trailing temperature, rainfall, vapor pressure and relative humidities with a delay of $9.37 \pm 0.02$ (standard error), $8.71 \pm 0.02,10.29 \pm 0.03$, and $18.05 \pm 0.24$ weeks respectively (see figure 4.4). Interestingly, when looking at the statistical association between dengue incidence and relative humidity for the sub-annual cycle, it appears that the time delay of dengue incidence compared to relative humidity decreases from 14.30 to 5.27 weeks over the 8 years of the study.

\subsection{Discussion and conclusion}

Using monthly aggregated data, Cuong et al. (2011) showed a clear annual cycle for dengue transmission in Hanoi from 1998 to 2009. In the present study, using weekly data allowed us to further characterize a sub-annual periodicity, in addition to the annual one. This sub-annual periodicity is reflected in the DF incidence time series, by a slow increase of incidence from the beginning of the year to the weeks 22-24 (June), followed by a faster increase of incidence until weeks 44-46 (November), which ends by a sharp decrease of incidence at the end of the rainy season. A potential drawback of working on weekly instead of monthly data is that it decreases the incidence values and thus increases the noise. However, given that we can still detect clear periodicities in our wavelet spectra, this does not seem to affect our analysis too much. When characterizing the reproductive ratio throughout the studied period, it displays, most of the years, two peaks per year, which is in accordance with the sub-annual periodicity of DF incidence. Among the climatic variables that we investigated (temperature, rainfall, relative humidities and vapor pressure), all of them expectedly displayed strong annual periodicities with temperature, rainfall and vapor pressure leading DF incidence by a constant delay of 8 to 10 weeks. In addition to this strong annual periodicity, relative humidity displays a sub-annual periodicity, as observed on DF incidence. The annual periodicity of relative humidity leads the annual periodicity of DF incidence by a constant delay of 18 weeks whereas the sub-annual periodicity of relative humidity leads the sub-annual periodicity of DF by a delay that decreases from 14.30 weeks in 2002 to 5.27 weeks in 2009 at an almost constant rate of 1.13 week per year. These 
(A)

(B)

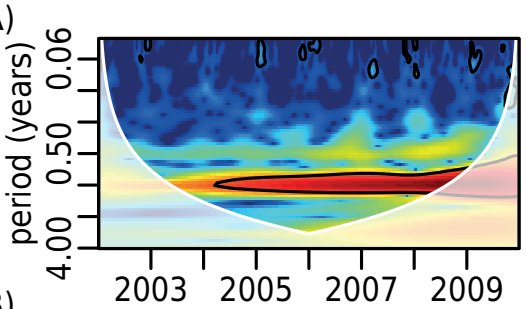

(C)

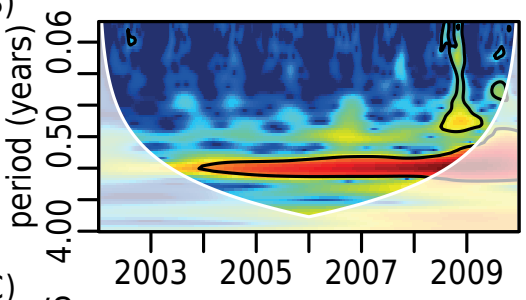

(D)
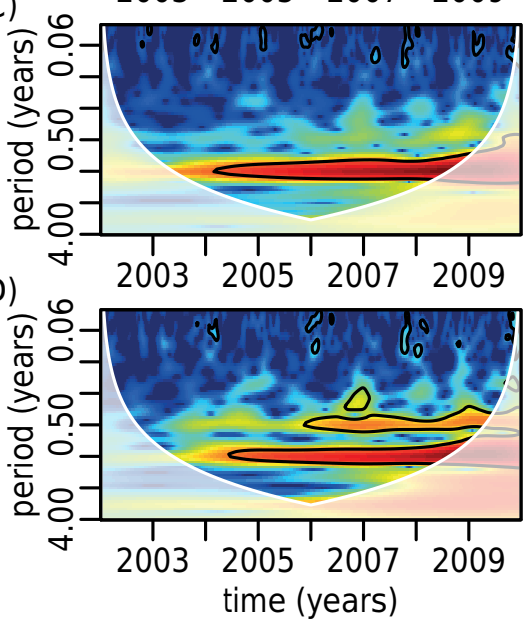
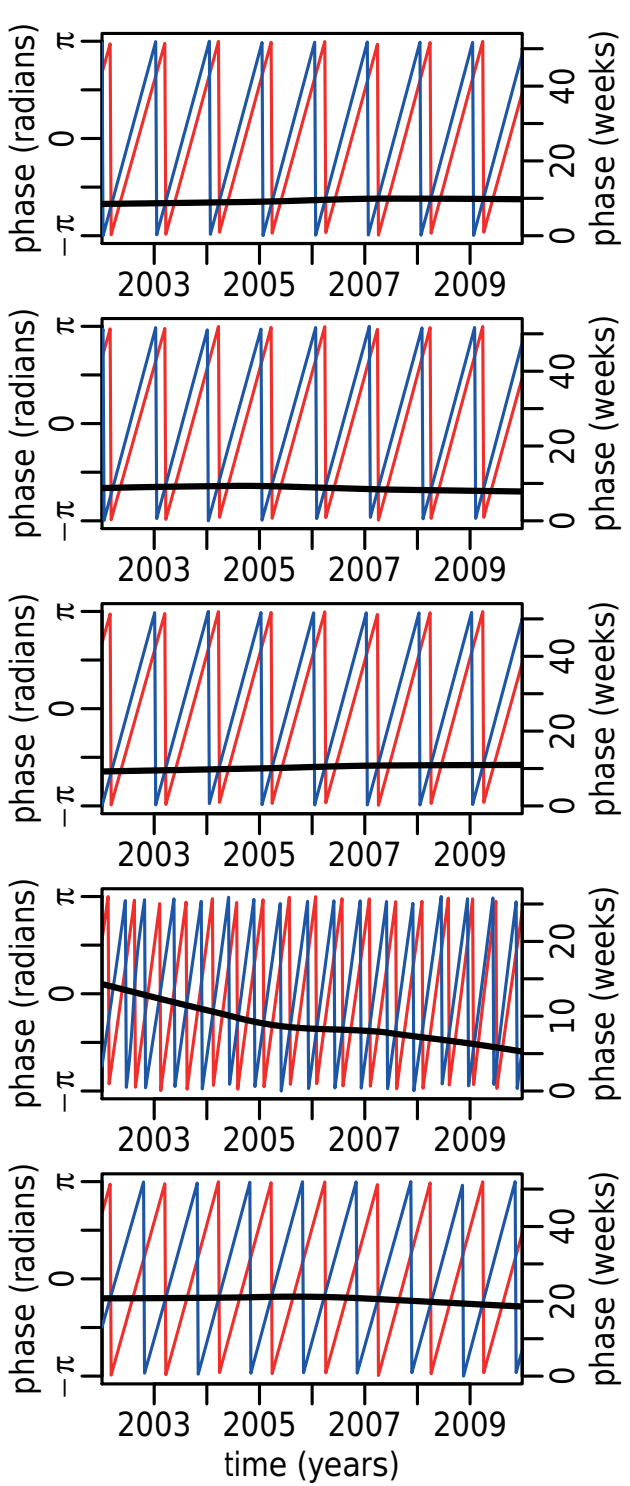

Figure 4.4: Cross-wavelet power spectra between DF and mean temperature (A), rainfall (B) and absolute (C) and relative (D) humidities in Hanoi from 2002 to 2009 (left column)

The right column shows the phase angles of the climatic variables (blue, left y-axis) and DF (red, left y-axis), as well as their difference (black, right y-axis). These phase angles are calculated on signals that have been filtered around the period of maximal power in the spectra of the left column, i.e. annual periodicity for all the climatic variables, as well as also the semi-annual periodicity for the relative humidity. In spectra of the left column, the black contour lines show the regions of power significant at the alpha-risk of 0.05 , the paled region of the spectrum delineates the cone of influence due to the zero-padding of the time series, and the power increases from dark blue to dark red 


\section{CLIMATIC-DRIVEN SEASONALITY OF EMERGING DENGUE FEVER IN HANOI, VIETNAM}

results are in general agreement with the findings of other studies that climatic factors play a role in the transmission cycles of DF. Interestingly, these two incidence peaks per year that we observed in Hanoi with periods of low incidence occurring in January and February (the coldest months in Hanoi) and in June and July (the warmest months in Hanoi) are in accordance with Eastin et al. (2014)'s observation in Columbia where they noted a significant decreases of DF cases soon after extended periods of either very cool or very hot temperatures. Likewise, a study in Taiwan found three turning points of DF that occurred around early August, late August/early September, and late October/early November. The first two turning points were shown to relate with two typhoons around early to mid $\mathrm{Au}$ gust in Taiwan that witnessed a sharp drop in temperature and substantial rainfall after it (Hsieh and Chen, 2009). Similarly, other studies in Thailand and Singapore also revealed significant associations between climatic variables and dengue incidence (Promprou et al., 2005a,b; Thu et al., 1998; Tipayamongkholgul et al., 2009; Wongkoon et al., 2011). For example, Tipayamongkholgul et al. (2009) conducted a study in the Gulf of Thailand and showed that the monthly average local relative humidity in the previous 3-6 months was negatively associated with epidemics of dengue and incidence of dengue cases. Wongkoon et al. (2011) in Chiang Rai, Thailand, showed that all climatic factors including minimum, maximum temperature, minimum and average relative humidity, evaporation, wind speed and rainfall lead increasing DF incidence by $0-2$ months. In our study, DF incidence is characterized by 2 quasi-cycles with a periodicity of 6 months with the first one showing a slow and constant increase and the second one showing a marked epidemic. The sharp rupture between these phases can be explained by the fact that the reproductive ratio is not constant throughout the year but actually exhibits two peaks per year, with the second peak at least as high as the first one. Given that dengue is vector-born, the factor limiting its transmission is either due to the mosquito population (mostly its population size), or the human population (mostly its proportion of susceptibles). Winter climatic conditions in Hanoi are not favorable to adult mosquitoes and most of the mosquito population survive the winter either as larvae or eggs (Higa et al., 2010). In the spring, when weather conditions become favorable again, eggs hatch and adults emerge, probably causing the first peak on the reproductive ratio and the consequent DF incidence increase. The second peak on the 


\section{CLIMATIC-DRIVEN SEASONALITY OF EMERGING DENGUE FEVER IN HANOI, VIETNAM}

reproductive ratio could be due to the second mosquito generation of the year (issued from the first one), hence its potential to be higher than the first one and even partially conceal it. This second peak of higher magnitude would be the cause of the epidemic peak observed on DF incidence during the second half of the year. This epidemic peak would thus be due more to an increase of the number of infected people than to an increase in the mosquito population size and the dengue reproductive ratio. Indeed, dengue epidemic peak appears even when the second peak on the reproductive ratio is not higher than the first one. Such a hypothesis to explain the mechanism of dengue epidemiology in Hanoi can be tested by collecting entomological data (larvae and adult densities estimates) in Hanoi all year-round and translating it into mathematical equations. This would allow to check whether a model based on this hypothesis can generate epidemiological patterns that are in accordance with the ones observed on DF incidence data. The 2 above-mentioned 6-month cycles observed on time series of DF incidence translate into the sub-annual periodicity that we have characterized in addition to the annual periodicity, with the annual periodicity of DF incidence mostly accounting for the high epidemic peak of the second half of the year, and the subannual periodicity mostly accounting for the slow and constant increase of DF incidence of the first half of the year. An interesting result of our analysis is that relative humidity also shows these two annual and sub-annual periodicities and that the sub-annual periodicity of relative humidity leads the sub-annual periodicity of DF incidence by a lag that decreases from 14.30 weeks in 2002 to 5.27 weeks in 2009. As interpreted above, this sub-annual periodicity reflects the first peak of the reproductive ratio that we interpreted in the paragraph above as the first mosquito hatching of the year. Explaining the observed shift in the timing of this first peak by a shift in mosquito hatching is biologically unrealistic. Alternatively, we propose that this shift is due to (i) the building-up of the human population immunity from year to year and (ii) the interactions between dengue serotypes (antibody-dependent enhancement, $\mathrm{ADE}$ ) as explained below. Most of primary dengue infections are asymptomatic (Bhatt et al., 2013). Before the emergence of dengue in Hanoi (in 2002), most of the human population may have been susceptible to the 4 dengue serotypes and hence most of the dengue cases may have been primary infections, most likely asymptomatic and thus unnoticed by the surveillance system. As the disease progressively emerges in Hanoi, population 


\section{CLIMATIC-DRIVEN SEASONALITY OF EMERGING DENGUE FEVER IN HANOI, VIETNAM}

immunity to different dengue serotypes increases, thus increasing the number of secondary infections relative to primary ones, and thus increasing the number of symptomatic detected cases. Expected consequences of this mechanism is not only an increase in the number of detected cases from year to year (as visible through the upward trend of DF incidence), but also an earlier detection of the epidemics. The latter would explain this observed shift in the sub-annual periodicity of the DF incidence. This mechanism can potentially be reinforced by some ADE-related mechanisms. Indeed, potential epidemiological consequences of the ADE hypothesis that have been proposed in the literature are that it increases the susceptibility to secondary infections and/or the transmissibility from individuals suffering from secondary infections (see for example (Recker et al., 2009). Thus, such a mechanism could also explain the number of detected cases from year to year, the earlier detection of the epidemic, and thus the shift in the sub-annual periodicity of the DF incidence mentioned above. Such a hypothesis could be tested by collecting immunological data from the human population of Hanoi (by an aged-stratified sero-prevalence survey for example) and investigating whether a mathematical model built on this hypothesis does generate the trend in DF incidence mean and timing that we observe on the data. Both DF incidence and relative humidity exhibit conspicuous annual and sub-annual periodicities and these periodicities happen to be strongly correlated. However, we warn against over-interpretation of such correlations in term of biological causation. One reason for such a caution in particular is that relative humidity is a variable that depends on both absolute humidity and temperature (the former being naturally strongly influenced by rainfalls). In case where absolute humidity (or rainfalls) and temperature are not perfectly correlated (which is most likely the case), we do expect that relative humidity exhibits annual and bi-annual periodicities, as the resultant of two periodic signals that are not perfectly in phase. Thus, instead of looking for a mechanistic link between DF incidence and relative humidity, it may be more relevant to look for two links: (i) one between DF incidence and absolute humidity and (ii) one between DF incidence and temperature, possibly accounting for a possible interaction between the two climatic variables. This particular point will be the topic of a subsequent study. In conclusion, our analysis on the links between climatic variables and DF incidence in Hanoi raises a number of questions of general interest on the relationships between climate and in- 


\section{CLIMATIC-DRIVEN SEASONALITY OF EMERGING DENGUE FEVER IN HANOI, VIETNAM}

fectious diseases epidemiology. Because of its highly seasonal climate (and thus potentially highly seasonal dengue transmission too), its important population size and density, and its dengue epidemiological transition (current emergence), Hanoi appears as the ideal set-up to test hypotheses about interaction between serotypes. This is an issue both under-understood and potentially of high relevance for vaccine development. Further investigations on dengue in Hanoi call for additional entomological and immunological data, as well as for theoretical developments. 


\title{
Chapter 5
}

\section{Risk factors associated with}

\author{
an outbreak of dengue
}

fever/dengue haemorrhagic

fever in Hanoi, Vietnam

This chapter has been published as: Toan DTT, Hoat LN, Hu W, Wright P and Martens $P$ (2014). Risk factors associated with an outbreak of dengue fever/dengue haemorrhagic fever in Hanoi, Vietnam. Epidemiol. Infect., Page 1 of 5. Cambridge University Press 2014. 


\section{RISK FACTORS ASSOCIATED WITH AN OUTBREAK OF DENGUE FEVER/DENGUE HAEMORRHAGIC FEVER IN HANOI, VIETNAM}

\subsection{Introduction}

In this chapter, I describe my work to identify which risk factors are associated with DF/DHF in patients admitted to hospitals in Hanoi. Hanoi is a major city in Vietnam with a population of approximately 9 million, and there has been a rapid population growth due to the influx of workers from rural areas. Some previous studies in Vietnam showed that people living in poorer regions and old tenement housing, including houses for transition workers tend to have generally unhygienic conditions and poor house design (e.g. lack of water supply, absence of window screens), both of which may promote Aedes mosquito proliferation. In 2008, a large province adjacent to Hanoi became a new district of Hanoi. The rapid movement of population between communities again recorded a largest outbreak of dengue for Hanoi (in 2009, the city recorded an almost 8,000 DF patients, representing a 15-fold increase from the previous year) (MOH, 2010; Thai et al., 2010). This study was carried out in three largest hospitals in Hanoi including the National Hospital of Tropical Diseases, the Hospital of Hanoi Medical University and the Infectious Diseases Department of Bach Mai Hospital. We performed a prospective matched case control study on patients who were diagnosed with DF/DHF; control patients were from the same hospital but negative for DF/DHF. We aim to measure the different association between case and control groups.

\subsection{Materials and methods}

Data collections have been carried out from August 2009 to March 2010. Approval from the hospital administration was obtained before approaching the patients. The criteria for notification of DF were based on the guidelines of the Ministry of Health, 1999, on surveillance, diagnosis and treatment of dengue. The guidelines stipulate that individuals are suspected to have dengue when they have acute febrile illness $\left(38^{\circ} \mathrm{C}\right)$ of $2-7$ days duration, with two or more of the following non-specific manifestations of DF: headache, retro-orbital pain, myalgia, arthralgia, rash, hemorrhagic manifestations, and leucopenia (MOH, 1999). The control group was matched to the study group for sex and age ( \pm 5 years). The characteristics of the $73 \mathrm{DF} / \mathrm{DHF}$ patients and the 73 control patients are shown in Table 5.1. 


\section{RISK FACTORS ASSOCIATED WITH AN OUTBREAK OF DENGUE FEVER/DENGUE HAEMORRHAGIC FEVER IN HANOI, VIETNAM}

All cases and control patients were interviewed during the period of hospitalization by two specially trained interviewers who administered a standard questionnaire. The questionnaire included information on demographic characteristics of the patients such as age, education level and occupation as well as variables related to their residence: location of house, type of house, source of water supply, and type of water containers in the house. The questionnaire had been pre-tested before being used in the study. Chi-square and Mann-Whitney tests were used to test for differences in demographic characteristics between DF/DHF patients and control patients. A multivariable backward stepwise logistic regression model was applied to identify the risk factors for DF/DHF. The independent variables consisted of: age, education, occupation, having been in an epidemic area within past 6 months; place of living: in urban or rural area, in rented house or near open sewer; and living conditions such as unhygienic environment, other family member had DF/DHF during past 6 months, presence of mosquitoes, presence of larvae in water containers, directly discharging sewage to ponds, and being flooded during the rainy season. The significance level was set at $\mathrm{p}<0.2$. Associations are expressed in terms of odds ratios (OR) with $95 \%$ confidence intervals. All analyses were performed using Stata statistical software, version 12.1.

\subsection{Results}

The characteristics of the $73 \mathrm{DF} / \mathrm{DHF}$ patients and 73 control patients are shown in Table 5.1.

The mean age of the case group and the control group were comparable. The distributions of education level and occupation were quite similar between the case and control groups; most of them had college education and many of them were students. Table 5.2 displays variables related to the domicile of the study population. In both groups, three quarters live in the inner districts of Hanoi.

More than half of the participants in the case group were living in rented houses, significantly higher than in control group, only one third. People in the DF/DHF group were living in smaller houses with a larger household size compared with those in the control group. Especially, a significantly higher proportion of people in the case group were living 


\section{RISK FACTORS ASSOCIATED WITH AN OUTBREAK OF DENGUE FEVER/DENGUE HAEMORRHAGIC FEVER IN HANOI, VIETNAM}

Table 5.1: Demographic characteristics of dengue fever/dengue haemorrhagic fever (DF/DHF) and control patients Characteristics.

\begin{tabular}{|c|c|c|c|c|}
\hline Characteristic & & DF/DHF $(n=73)$ & Control $(n=73)$ & $P$ \\
\hline \multirow[t]{2}{*}{ Sex } & Male & $36(49.32 \%)$ & $36(49.32 \%)$ & \\
\hline & Female & $37(50.68 \%)$ & $37(50.68 \%)$ & $>0.05^{*}$ \\
\hline \multirow[t]{2}{*}{ Age (years) } & Mean \pm SD & $28.1 \pm 9.4$ & $27.4 \pm 9.9$ & $>0.05+$ \\
\hline & Range & $17-75$ & $18-78$ & \\
\hline \multirow[t]{4}{*}{ Education } & Elementary school & 9 (12.3\%) & $10(13.7 \%)$ & \\
\hline & Middle school & $10(13.7 \%)$ & $4(5.5 \%)$ & $>0.05^{*}$ \\
\hline & High school & $11(15.1 \%)$ & $6(8.2 \%)$ & \\
\hline & University/College & 43 (58.9\%) & $53(72.6 \%)$ & \\
\hline \multirow[t]{5}{*}{ Occupation } & Office worker & $17(23.3 \%)$ & 24 (32.9\%) & $>0.05^{*}$ \\
\hline & Business person & $7(9.6 \%)$ & $12(16.4 \%)$ & \\
\hline & Home worker & $4(5.5 \%)$ & $7(9.6 \%)$ & \\
\hline & Pupil/student & $36(49.3 \%)$ & $22(30.1 \%)$ & \\
\hline & Other & 9 (12.3\%) & $8(11.0 \%)$ & \\
\hline
\end{tabular}

* By chi-square test

† By Mann-Whitney test 


\section{RISK FACTORS ASSOCIATED WITH AN OUTBREAK OF DENGUE FEVER/DENGUE HAEMORRHAGIC FEVER IN HANOI, VIETNAM}

Table 5.2: Housing variables of dengue fever/dengue haemorrhagic fever (DF/DHF) and control patients Variables.

\begin{tabular}{|c|c|c|c|c|}
\hline \multicolumn{2}{|l|}{ Variables } & $\begin{array}{c}\text { DF/DHF } \\
(n=73)\end{array}$ & $\begin{array}{l}\text { Control } \\
(n=73)\end{array}$ & $p$ \\
\hline \multirow[t]{2}{*}{ Living area } & Inner district & $54(74.0 \%)$ & $55(75.3 \%)$ & $>0.05^{*}$ \\
\hline & Outer district & $19(26.0 \%)$ & $18(24.7 \%)$ & \\
\hline \multirow[t]{2}{*}{ Accommodation ownership } & Rented & $41(56.2 \%)$ & $25(34.3 \%)$ & $<0.05^{*}$ \\
\hline & Owned & $32(43.8 \%)$ & $48(65.7 \%)$ & \\
\hline \multirow[t]{5}{*}{ Type of housing } & Solid house & $43(58.9 \%)$ & 35 (47.9\%) & \\
\hline & Temporary house & $7(9.6 \%)$ & $7(9.6 \%)$ & \\
\hline & Old condominium & $11(15.1 \%)$ & $15(20.6 \%)$ & $>0.05^{*}$ \\
\hline & New condominium & $0(0.0 \%)$ & $3(4.1 \%)$ & \\
\hline & Other & $12(16.4 \%)$ & $13(17.8 \%)$ & \\
\hline \multirow[t]{2}{*}{ Area of household $\left(\mathrm{m}^{2}\right)$} & Mean \pm SD & $54.1 \pm 47.1$ & $68.2 \pm 69.9$ & $>0.05+$ \\
\hline & Range & $8-200$ & $9-350$ & \\
\hline \multirow[t]{2}{*}{ Number of people in household } & Mean \pm SD & $4.2 \pm 2.3$ & $4.9 \pm 4.3$ & $>0.05+$ \\
\hline & Range & $1-10$ & $1-19$ & \\
\hline \multirow[t]{2}{*}{ House with water storage containers } & $\begin{array}{l}\text { Yes (water tank } \\
\text { without cover) }\end{array}$ & $22(10.7 \%)$ & $21(1.3 \%)$ & $>0.05^{*}$ \\
\hline & No & $53(70.8 \%)$ & $54(72.0 \%)$ & \\
\hline \multirow{3}{*}{$\begin{array}{l}\text { House with water storage containers } \\
\text { infested with larvae and/or pupae }\end{array}$} & Yes & $11(15.1 \%)$ & $13(17.8 \%)$ & $>0.05^{*}$ \\
\hline & No & $52(71.2 \%)$ & $56(76.7 \%)$ & \\
\hline & Don't know & $10(13.7 \%)$ & $4(5.5 \%)$ & \\
\hline \multirow[t]{3}{*}{ House environment } & Pond/ lake/ river & $16(19.3 \%)$ & $21(24.1 \%)$ & $>0.05^{*}$ \\
\hline & Open sewer & $33(39.7 \%)$ & $13(14.9 \%)$ & $<0.01^{*}$ \\
\hline & $\begin{array}{l}\text { Garbage collection } \\
\text { point }\end{array}$ & $7(36.8 \%)$ & $12(63.2 \%)$ & $>0.05^{*}$ \\
\hline
\end{tabular}

* By chi-square test

† By Mann-Whitney test 


\section{RISK FACTORS ASSOCIATED WITH AN OUTBREAK OF DENGUE FEVER/DENGUE HAEMORRHAGIC FEVER IN HANOI, VIETNAM}

in homes are near suitable breeding areas for mosquitoes, such as open sewers.

Table 5.3: crude and adjusted logistic regression odds ratios on selected variables.

\begin{tabular}{|c|c|c|c|}
\hline Independent variables & Grouping & Crude odds ratios (95\% CI) & $\begin{array}{l}\text { Logistic regression } \\
\text { (adjusted) odds ratios } \\
(95 \% \mathrm{CI})\end{array}$ \\
\hline Living in rented house & Yes & $2.2(1,1-4,8)$ & $2.2(1,1-4,6)$ \\
\hline $\begin{array}{l}\text { Other family member had DF/DHF } \\
\text { during past } 6 \text { months }\end{array}$ & Yes & $2(0,9-4,6)$ & $1.96(0.9-4.3)$ \\
\hline Unhygienic house & Yes & $3,3(0,9-12)$ & $3,4(1-11,7)$ \\
\hline Mosquitoes in house & Yes & $6,8(0,7-64,2)$ & $6,3(0,7-59,5)$ \\
\hline Near open sewer & Yes & $6,9(0,7-67,1)$ & $7,9(0,9-71,9)$ \\
\hline $\begin{array}{l}\text { Discharging sewage directly into } \\
\text { ponds }\end{array}$ & Yes & $5,9(1,3-26,4)$ & $4,3(1,1-16,9)$ \\
\hline
\end{tabular}

The analysis revealed that living in rental house, living near open sewers and untreated water going directly to nearby ponds/lakes were all significantly associated with DF/DHF. Living in a rented house increased risk by 2.2 times $(\mathrm{OR}$ adjusted $=2.2,95 \% \mathrm{CI}=1.1-$ 4.6). Living in an unhygienic house or one directly discharging sewage to ponds increased risk by 3.4 times and 4.3 times, respectively ( $\mathrm{OR}$ adjusted $=3.4,95 \% \mathrm{CI}=1-11.7$; OR adjusted $=4.3,95 \% \mathrm{CI}=1.1-16.9$ ). Detecting mosquitoes in the house or living near an open sewer constituted a very high risk ( $\mathrm{OR}$ adjusted $=6.3,95 \% \mathrm{CI}=0,7-59$; and $\mathrm{OR}$ adjusted $=6.9,95 \% \mathrm{CI}=0.9-71.9$ respectively), but the difference between the case and control groups was not significant.

\subsection{Discussion}

The results of this study revealed that DF/DHF in Hanoi appeared mainly in downtown districts and much less in suburban districts, which is consistent with the statistical reports on the DF epidemic in 2009. The highest morbidity rate was found in the age group 15 to 30 years; at higher ages the rate became very low. Dung and Cam reported in 2003 that more 


\section{RISK FACTORS ASSOCIATED WITH AN OUTBREAK OF DENGUE FEVER/DENGUE HAEMORRHAGIC FEVER IN HANOI, VIETNAM}

than $90 \%$ of dengue morbidity was between ages 15 and 25 (Dung and Cam, 2005). This could be related to differences in lifestyle, time spent outdoors near vectors, sleeping without mosquito nets or other aspects of inadequate disease prevention among the young. Another explanation could be that the disease can create lifelong immunity for the individual, so that older persons who have been exposed more often may have more resistance, decreasing the morbidity rate. Considering how many cases were among young people, it is perhaps not surprising that around half of the positive cases were among students. The second dominant group was office workers. This result differs from the result of a study in Kamphaeng Phet, Thailand (Koenraadt et al., 2006) where $65 \%$ of the patients were farmers. The discrepancy is probably due to a different job distribution in the two regions studied. Students may also be prominent among the cases in our study because many of them live in rental accommodation, which was another risk factor for dengue. The combination of cramped rented living spaces, an unsanitary environment, infrequent cleaning, little knowledge of dengue fever, and little interest in prevention activities may explain why the morbidity rate in the rental group is rising. People living near stagnant water like ponds, lakes and river or open sewers, or favorable mosquito breeding places like garbage collection points, had higher rates of morbidity. This is consistent with other national and international studies (Be, 2005; Heukelbach et al., 2001; Huong and et Al., 2009; Itrat et al., 2008; Nguyen, 1999; Syed et al., 2010; Toan et al., 2013). In Brazil (Heukelbach et al., 2001) the epidemic was associated with proximity to uncontrolled waterways and stagnant water in tanks, gutters, and cans. In Pakistan, poor condition of the house, such as uncovered toilet water tank or leaking water pipes, was a highly significant risk factor for the presence of Aedes foci (Itrat et al., 2008). In Vietnam, a study in Binh Thanh, Dong Thap (Huong and et Al., 2009) found that while most families stored their drinking water, only three quarters of them covered their water storage containers. Nowadays, although Hanoi residents seldom use water storage containers like jars or pots, the large area of natural water surfaces available makes an annual epidemic almost unavoidable (Nga, 1992). Because dengue-spreading mosquitoes spend three stages of their life-cycle in water, it is recommended to reduce mosquito living sites like long-term stagnant water storage inside and outside the house, open sewers, and natural water surface. According to around half of the respondents, their homes and surrounding environments are 


\section{RISK FACTORS ASSOCIATED WITH AN OUTBREAK OF DENGUE FEVER/DENGUE HAEMORRHAGIC FEVER IN HANOI, VIETNAM}

not really clean. Although the difference between case and control groups was not statistically significant, it did reflect how the unsanitary, polluted living environment in the modern environment of the capital is favorable for the vector and the disease. Environmental factors have a powerful influence on the appearance of DF/DHF. Simple interventions could help; in our study, people living in houses with uncovered water tanks were 7.9 times more likely to get DF. Those in houses discharging sewage directly discharging into ponds had a 5.9 times higher risk than those in houses with sanitary sewage systems. For civil authorities it is important to note that people who stay in rental accommodation had double the risk of those owning their homes. These influences of the environmental factors have also been found in other studies. A 2001 study in Brazil revealed that people living in a slum area had a nearly 10 times higher risk of dengue fever (Heukelbach et al., 2001). In An Giang, Vietnam, people living in the homes with gardens or water tanks had a three-fold higher risk (Be, 2005). This study was conducted soon after Hanoi had experienced heavy flooding in August, 2009 which reduced the selection and recall bias often found in a case-control study. However, our data came from the three major hospitals for infectious diseases in Hanoi, and may have missed factors related to cases that did not present in them. Our logistic regression model was set up to identify factors that may influence dengue transmission dynamics. In a complex epidemiological setting, transmission dynamics involve interactions among people, dengue viruses, vectors and ecosystems, where biotic and abiotic determinants have both direct and indirect influences on transmission. Our model cannot account for all possible variables. More information is needed to further unravel the predictors of dengue transmission in Hanoi, which will help to inform planning of prevention and control measures.

\subsection{Conclusion}

This integrated analysis of the eco-social determinants of DF transmission risk contributes to improve understanding of the dynamics of dengue transmission in Hanoi. Our study shows various well-known risk factors of DF which are components of human behaviour, can be addressed through modifications of municipal services. The social and demographical 
5. RISK FACTORS ASSOCIATED WITH AN OUTBREAK OF DENGUE FEVER/DENGUE HAEMORRHAGIC FEVER IN HANOI, VIETNAM

contexts and lifestyles of people in Hanoi will significantly influence transmission and all information on them will support dengue prevention and control programs. 


\section{Chapter 6}

\section{ENVIRONMENTAL FACTORS}

\section{INFLUENCING EPIDEMICS OF}

\section{DENGUE FEVER IN VIETNAM}

This chapter has been written by: Do Thi Thanh Toan, Bram Oosterbroek, Pham Quang Thai, Pamela Wright, and Martens Pim (May, 2015).Submitted to Asia Pacific Journal of Tropical Medicine on July, $3^{\text {rd }}$, 2015 


\section{ENVIRONMENTAL FACTORS INFLUENCING EPIDEMICS OF DENGUE FEVER IN VIETNAM}

\subsection{Introduction}

Dengue is a re-emerging infectious disease that mainly occurs in the tropical and subtropical regions around the world (WHO, 2009). Urban and suburban environments in those regions are particularly fragile due to rapid population growth (e.g. massive influx of workers from rural areas, causing unorganized urbanization) and the abundance of potential breeding sites (Braga et al., 2010; Getis et al., 2003; Honório et al., 2009; Paz-Soldan et al., 2011; Teixeira et al., 2002). However, with the changes of other global drivers such as pollution, land use patterns, urbanization and human mobility, dengue fever is now spreading outside the humid tropical and sub-tropical urban centers where it has been common (Hay et al., 2002; Jacobs et al., 2005; Knudsen, 1995). Increasing incidence of dengue adversely impacts the health, social and economic status of all the affected countries by causing a significant loss of life as well as debilitation, creating a major economic burden on society (Shepard et al., 2013).

Dengue virus occurs in four virus serotypes: DENV-1, DENV-2, DENV-3, DENV-4; it belongs to the genus Flavivirus (family Flaviridae). Infection with dengue virus causes a wide range of clinical symptoms, ranging from a mild, undifferentiated fever, to classical dengue fever (DF) or dengue (also known as break bone fever because of the pain it causes in joints), through to dengue hemorrhagic fever (DHF) and dengue shock syndrome (DSS). During the course of a lifetime, one person can be infected with all four of these immunologically distinct serotypes at different times (Adams and Boots, 2006; Coudeville and Garnett, 2012; Hartley et al., 2002; Murray et al., 2013). A combination vaccine against all serotypes of dengue virus is still not available. Thus, while waiting for an effective vaccine, the only way to prevent dengue transmission is to reduce the population of its principal vector, Aedes aegypti. However, that goal is also characterized by a lack of success and sustainability (Chadee et al., 2005; Halmar and Peter, 2011; McConnell and Gubler, 2003).

Dengue was first reported in Vietnam in 1958 (Tien et al., 1999). Since then the number of infections has steadily increased until now Vietnam has among the worlds highest numbers of dengue cases. Dengue infection is now endemic in many parts of Vietnam. According to Ministry of Health statistics, hyperendemics have been reported at a regular interval of 6-10 


\section{ENVIRONMENTAL FACTORS INFLUENCING EPIDEMICS OF DENGUE FEVER IN VIETNAM}

years, with peaks in 1983, 1987, 1998 and 2009 (MOH, 2010). In these years, Vietnam also experienced an increase of El Niño activity.

Generally, the majority of dengue cases have been reported from Central to Southern Vietnam (Vu et al., 2005). However, in recent years, there has been an upward trend of dengue fever in all areas of Vietnam including the Northern provinces, in which Hanoi recorded the highest incidence rate of dengue fever in an urban center (GSO, 2008). In 2009, the city recorded almost 16,000 DF patients, registered in most of its districts. That number was 15 times higher than the previous year 2008, while in the past ten years, the number of dengue patients in Hanoi was always at the lowest level in the country.

Several previous reports have described how environmental factors such as human population dynamics and their effects on exposure risk, changes in vegetation, hydrology and other landscape features have an indirect impact on the trend of dengue transmission and distribution pattern (Abdul Aziz and Abdullah Rusli, 2012; Huong and et Al., 2009; Toan et al., 2014; Umor et al., 2008). For example, our previous study has shown that people living near stagnant water like ponds, lakes and river or open sewers, or favorable mosquito breeding places like garbage collection points, had higher rates of morbidity with dengue fever (Toan et al., 2014). However, the roles of population density and environmental factors (for example water surface and road length) as risk factors for dengue fever are still poorly understood. In this study, we apply geographic information systems (GIS) techniques to map the risk of dengue fever in relation to water surface, road length and areas of differing population density. We will also test for the association between population density and the risk of dengue fever by using the Ordinary Least Squares (OLS) linear regression model.

\subsection{Methodology}

\subsubsection{Study site}

Hanoi, before it merged with parts of neighbouring provinces in 2008), had 14 districts, now known as old Hanoi (Toan et al., 2014). This geographic area of old Hanoi was selected as a study site because it made it possible to use consistent data from time periods before 


\section{ENVIRONMENTAL FACTORS INFLUENCING EPIDEMICS OF DENGUE FEVER IN VIETNAM}

and after the year 2008. All GIS analyses reported here were performed on the National Institute of Hygiene and Epidemiology (NIHE) dengue case data, which falls within the geographic boundaries of this study site, as well as environmental data from within the same geographic boundaries.

\subsubsection{Data sources}

Data on all of the dengue cases from the National Institute of Hygiene and Epidemiology dataset (NIHE) were used to perform all GIS analyses described in this paper. This dataset contains annual data on DF cases from January 2007 to December 2011. It includes all cases reported to the Hanoi city network of commune health stations located in each village all over the city. Case locations are recorded as the street addresses of patients diagnosed as having dengue fever. GPS information was then collected according to each patients address by using a Garmin GPS60 (Garmin Corporation, Taipei County, Taiwan) global positioning system while doing a field survey. A map with communes, population count per commune, and maps showing water bodies and roads were obtained from the Department of Survey and Mapping of Vietnam in 2010.

\subsubsection{Data analysis}

Geographic Information System software (ArcGIS 10.2) was used to calculate incidence rates, perform a proximity analysis (information about the distance between dengue cases and selected factors) and to relate environmental data per commune to dengue cases. The year 2009 was analyzed separately because in that year, the incidence rate of dengue fever was the highest ever recorded. The difference between that years incidence rate and those of the two-year periods 2007-2008 and 2010-2011 can be called dramatic (see Table 6.1). It was probably caused by the long and heavy rainfall during the last days of October and the first week of November 2008 which resulted in the most devastating flood in Hanoi since 1973 (Bich et al., 2011).

\section{Incidence rate}

All analyses were performed on maps displaying incidence rates (dengue cases per 100 


\section{ENVIRONMENTAL FACTORS INFLUENCING EPIDEMICS OF DENGUE FEVER IN VIETNAM}

Table 6.1: Total number of cases according to years.

\begin{tabular}{lc}
\hline Year & Total number of cases \\
\hline 2007 & 1547 \\
2008 & 1622 \\
2009 & 14039 \\
2010 & 2921 \\
2011 & 4343 \\
All years & 24472 \\
\hline
\end{tabular}

000 population per year) per commune. These maps were created by geo-referencing the dengue cases, summing up the number of cases per community and then dividing this total number of cases per community by the total population count per community.

\section{Proximity Analysis water bodies}

The number of cases per $\mathrm{km}^{2}$ within a zone of 50 meters and a zone of 100 meters around water bodies was calculated, in order to compare with the numbers of cases per $\mathrm{km}^{2}$ further away from water bodies.

\section{Proximity Analysis roads}

The number of cases per $\mathrm{km}^{2}$ within a zone of 50 meters around roads was calculated to compare this number with the number of cases per $\mathrm{km}^{2}$ further away from roads.

\section{Factors per commune}

Data about surface water and infrastructure were available in medium-high spatial resolution in the form of maps containing individual water bodies and roads. Population count was available per commune as highest spatial resolution for the 229 communes in 'old Hanoi'. The total surface area of water bodies and total length of roads per commune was calculated. This was performed by adding up the areas of water bodies and lengths of roads that fell completely within the commune borders, as well as the areas and lengths of all water body and road parts that fell within those commune borders. Subsequently, both total water surface area per commune and total road length per commune were divided by total surface area per commune. Population density per commune was simply calculated by dividing population numbers per community by the total surface area of each commune. This allowed for comparison with the dengue incidence rate per commune. Moreover, to 


\section{ENVIRONMENTAL FACTORS INFLUENCING EPIDEMICS OF DENGUE FEVER IN VIETNAM}

test for the spatial association between population density and the incidence of dengue, we performed Ordinary Least Squares (OLS) linear regression. The model was set as: $\mathrm{IR}=\beta_{0}$ $+\beta_{1} \mathrm{POP}_{\text {dens }}+\varepsilon$, where $\beta_{0}$ and $\beta_{1}$ are the regression coefficients, POPdens is population density and $\varepsilon$ is the model random error. All analyses were performed using the ArcGIS 10.02 program.

\subsection{Results}

\subsubsection{Distribution of cases in relation to distance from a body of water}

The total number of connected water bodies within our study site was 1832. A connected water body is defined as a water body that visually might be seem to consist of several components (for example a river and a stream), but of which the water surfaces are connected. Water body types in our dataset included rivers, lakes, ponds and canals.

Table 6.2 and Figure 6.1 show the distances of cases from the closest water body during the three time periods. In the period 2007-2008, the average distance of dengue cases from the closest water body was $188142 \mathrm{~m}$. Half of the cases were located within a distance of under $173 \mathrm{~m}$.

Table 6.2: Distance of cases from water bodies for three time periods.

\begin{tabular}{lccc}
\hline & $2007-2008$ & 2009 & $2010-2011$ \\
\hline Count & 3169 & 14039 & 7264 \\
Minimum & $0 \mathrm{~m}$ & $0 \mathrm{~m}$ & $0 \mathrm{~m}$ \\
Maximum & $1605 \mathrm{~m}$ & $1597 \mathrm{~m}$ & $1140 \mathrm{~m}$ \\
Mean & $188 \mathrm{~m}$ & $197 \mathrm{~m}$ & $213 \mathrm{~m}$ \\
Standard deviation & $142 \mathrm{~m}$ & $150 \mathrm{~m}$ & $163 \mathrm{~m}$ \\
\hline
\end{tabular}

In 2009, the average distance of dengue fever from the closest water body was $197 \pm 150$ $\mathrm{m}$, and more than half of the cases were within the radius of $146 \mathrm{~m}$. The average distance of dengue cases from the closest water body in 2010-2011 was $213 \pm 163 \mathrm{~m}$ and more than half of the cases were within $224 \mathrm{~m}$ of the water. The distribution of dengue cases in this 


\section{ENVIRONMENTAL FACTORS INFLUENCING EPIDEMICS OF DENGUE FEVER IN VIETNAM}

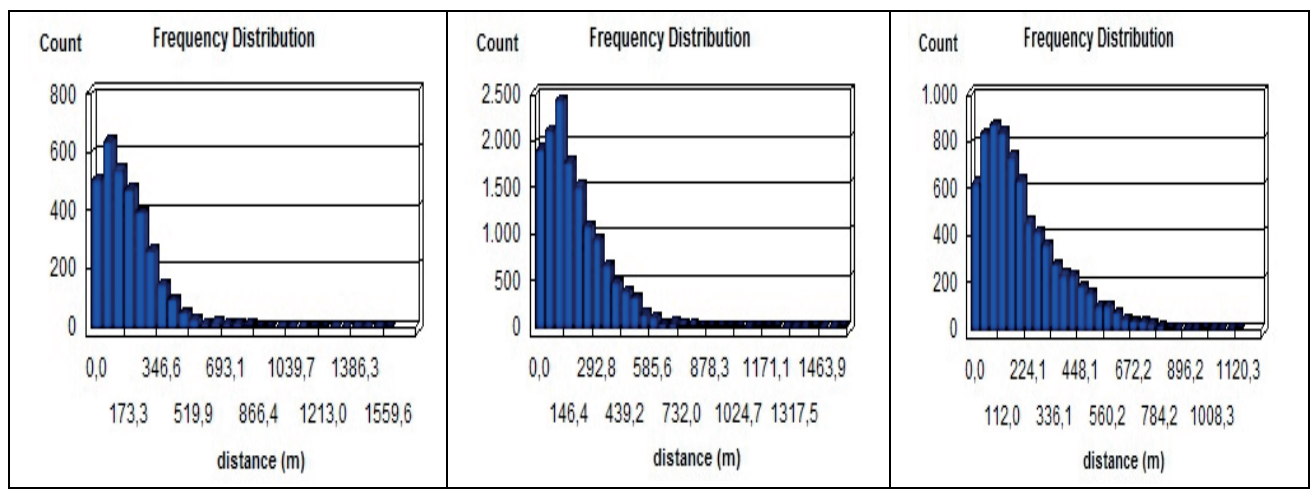

Figure 6.1: Frequency distribution of the case water body distance for three time periods.

time period was more dispersed than in the previous year even though the total number of cases was lower.

Table 6.3 shows the density of dengue cases - number of cases per $\mathrm{km}^{2}$ - within a zone of 50 and a zone of $100 \mathrm{~m}$ around water bodies. The highest density appeared between 50-100 m from the waters edge. Most water bodies in Hanoi have either parkland or roads or both around them, so that housing density is low very close to the water bodies. The correlation between proximity to water surface and dengue transmission is not very strong; more detailed data on population density in each area around the water surfaces would be needed for clear interpretation of this data.

Table 6.3: Cases per $\mathrm{km}^{2}$ close to water bodies.

\begin{tabular}{lccccc}
\hline Year & $<100 m$ & $>100 m$ & $<50 m$ & $>50 m$ & $50-100 m$ \\
\hline $2007-2008$ & 3,627 & 3,179 & 2,212 & 3,618 & 6,438 \\
2009 & 14,947 & 14,538 & 10,868 & 15,770 & 23,041 \\
$2010-2011$ & 7,473 & 7,627 & 4,811 & 8,354 & 12,756 \\
All years & 26,048 & 25,345 & 17,890 & 27,742 & 42,235 \\
\hline
\end{tabular}




\section{ENVIRONMENTAL FACTORS INFLUENCING EPIDEMICS OF DENGUE FEVER IN VIETNAM}

\subsubsection{Distance of cases from nearest roads}

In this dataset, we included all roads larger than $10 \mathrm{~m}$ wide, part of the roads between $10 \mathrm{~m}$ and $6 \mathrm{~m}$ wide and none of the roads smaller than $6 \mathrm{~m}$ wide. The total length of roads in our dataset was $1433 \mathrm{~km}$. Table 6.4 shows that more cases were found living within a zone of $50 \mathrm{~m}$ around the main roads of Hanoi than further away from that zone.

Table 6.4: Cases per $\mathrm{km}^{2}$ close to roads.

\begin{tabular}{lcc}
\hline Year & $<50 \mathrm{~m}$ & $>50 \mathrm{~m}$ \\
\hline $2007-2008$ & 10,554 & 2,280 \\
2009 & 55,650 & 8,711 \\
$2010-2011$ & 30,355 & 4,263 \\
All years & 96,559 & 15,255 \\
\hline
\end{tabular}

In Figure 6.2, the association with roads is clearly visible, especially in the peripheral areas. It should be noted that the number of people living within $50 \mathrm{~m}$ of the main roads is more than six times higher than those living outside the range of $50 \mathrm{~m}$.

\subsubsection{Risk factors for appearance of dengue cases per com- mune}

The maps in Figure 6.3 suggest that the incidence rate in different communes might be correlated to population density and road length. We found that in the areas with higher density of population, and higher street length ratio, there was a higher rate of dengue incidence. Those areas were found in the North and Center of the city. Although an association with water might be expected, the lower population density close to water bodies might have complicated the interpretation of that data.

OLS regression analyses of dengue incidence covering the 229 communes were initially performed for each time period and are shown in Table 6.4. The results revealed that population density was significantly associated with dengue incidence. The positive coefficient on the dengue incidence in all three time periods for which the analysis was done indicates that communes with dengue had higher population density than communes without dengue. The 


\section{ENVIRONMENTAL FACTORS INFLUENCING EPIDEMICS OF}

DENGUE FEVER IN VIETNAM

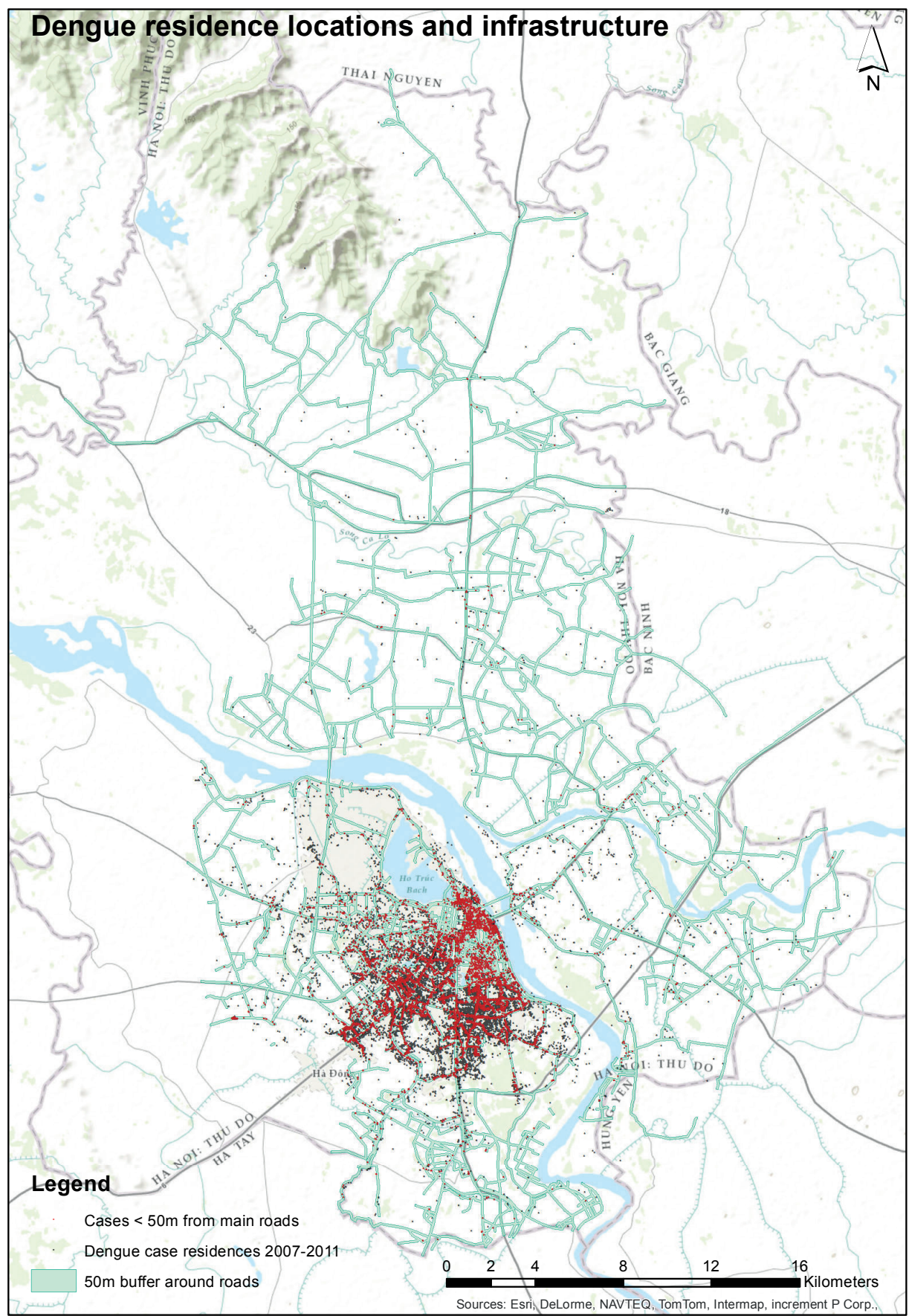

Figure 6.2: Dengue cases close to main roads. 


\section{ENVIRONMENTAL FACTORS INFLUENCING EPIDEMICS OF DENGUE FEVER IN VIETNAM}

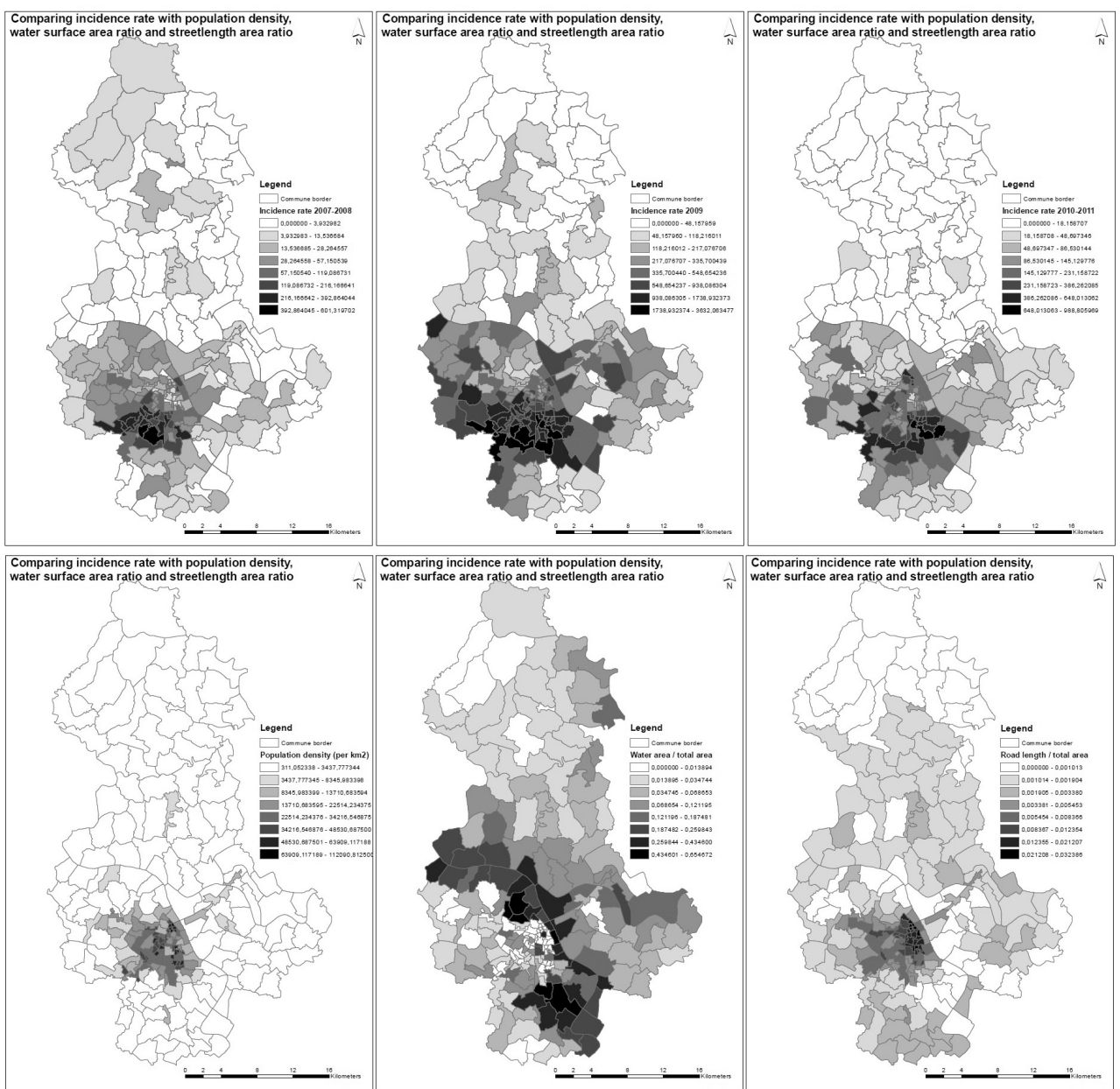

Figure 6.3: Maps of dengue incidence rate, population density, street length area ratio and water surface area ratio.

(from left to right) 


\section{ENVIRONMENTAL FACTORS INFLUENCING EPIDEMICS OF DENGUE FEVER IN VIETNAM}

year 2009 had the highest coefficient $(0.0046$; se=0.0010), which means that communes with dengue had 4.6 higher population density than communes without dengue. This regression model explained $7.4 \%$ of the total variance of DF. However, in the period 2010-2011, the model explained $17 \%$ of the total variance of $\mathrm{DF}$.

Table 6.5: Ordinary Least Squares (OLS) results.

\begin{tabular}{|c|c|c|c|}
\hline Parameter & Estimated Value & Standard Error & $p$-value \\
\hline \multicolumn{4}{|c|}{ Year 2007-2008 } \\
\hline Intercept & 23.477519 & 5.153337 & $0.000011^{*}$ \\
\hline POPdens & 0.000699 & 0.000167 & $0.000045^{*}$ \\
\hline Adjusted $\mathrm{R}^{2}$ & 0.067643 & & \\
\hline $\mathrm{AIC}$ & 2543.323 & & \\
\hline \multicolumn{4}{|c|}{ Year 2009} \\
\hline Intercept & 260.600755 & 32.253632 & $0.000000^{*}$ \\
\hline POPdens & 0.004585 & 0.001045 & $0.000021^{*}$ \\
\hline Adjusted $\mathrm{R}^{2}$ & 0.074105 & & \\
\hline AIC & 3383.289 & & \\
\hline \multicolumn{4}{|c|}{ Year $2010-2011$} \\
\hline Intercept & 54.23406 & 9.143114 & $0.000000^{*}$ \\
\hline POPdens & 0.002047 & 0.000296 & $0.000000^{*}$ \\
\hline Adjusted $\mathrm{R}^{2}$ & 0.170148 & & \\
\hline AIC & 2805.921 & & \\
\hline
\end{tabular}

\subsection{Discussion}

In a previous study in Hanoi (Toan et al., 2014), we found that certain environmental factors contributed to the expansion of dengue transmission, such as people living near bodies of stagnant water, and people living near places favorable for mosquito breeding. The results of this study again illustrated the contribution of environmental factors to dengue transmission, including proximity to water surfaces and roads.

Our results demonstrated that the number of dengue cases per $\mathrm{km}^{2}$ within a zone of 50 $\mathrm{m}$ around the main roads of Hanoi was higher than farther away from roads. Of course, the population density is also higher near the roads, so a higher number of cases would be expected. Hanoi currently has nearly $7500 \mathrm{~km}$ of transportation roads, accounting for $6.8 \%$ of the city area, which is equivalent to a road area of $11 \mathrm{~m}^{2}$ per person. Aedes 


\section{ENVIRONMENTAL FACTORS INFLUENCING EPIDEMICS OF DENGUE FEVER IN VIETNAM}

aegypti is known as living in close proximity to human hosts, inside or around residential areas. According to WHO (1986), the flight range of A. aegypti is very limited (50-100 m). Consequently, dengue transmission may rely heavily upon either infected mosquitoes being transported from one region to another and/or on movement of infected persons. Therefore the proximity to transportation arteries such as major roadways may be an important factor in the geographical spread of dengue.

Hanoi is also known as a city of ponds and lakes, where water covers approximately 756 hectares and each 0.5 kilometer of water surface equals to one square kilometer of land area. With a large area of natural water surfaces available, an annual dengue epidemic is almost unavoidable (Nga, 1992). This result was also revealed in our study when we found that the density of dengue cases (number of cases per $\mathrm{km}^{2}$ within a zone of 50 to $100 \mathrm{~m}$ around water bodies of Hanoi was higher than farther away from water. The area less than $50 \mathrm{~m}$ from water bodies is often occupied by parkland or roadways, so the population density there is relatively low. Several studies in Taiwan also found that areas along the coastal lines, ports, water bodies, or transportation arteries tended to witness more DF cases (Chadee et al., 2007; Chowell et al., 2008; Lin et al., 2006). For example in Kaoshiung City in Taiwan, Hsueh et al. (2012) demonstrated that $75 \%$ of DF cases occurred within $1.5 \mathrm{~km}$ of a river, and within $1.0 \mathrm{~km}$ of a major road.

The association of dengue fever not only with areas of low land topography, but also with areas having high population density has been reported from many countries including not only Vietnam but also Malaysia and Taiwan. In Vietnam, especially in a big city like Hanoi, the population density is very high and most of the people live along the roads. In some inner districts of Hanoi the population density even reach to nearly 30.000 people $/ \mathrm{km}^{2}$, such as Dong Da District or Hai Ba Trung District. Under these conditions, Aedes aegypti mosquitoes do not have to travel far to find victims. From our maps, we found that there is a significant association between population density and occurence of dengue fever in Hanoi. Most of the cases were found concentrated in the North and Center of the city where all six central districts with the highest population density are located. In addition, the high immigration rate into Hanoi recently, especially in the six central districts could explain the increased variance of dengue according to population density. Another report on a 


\section{ENVIRONMENTAL FACTORS INFLUENCING EPIDEMICS OF DENGUE FEVER IN VIETNAM}

study in Khanh Hoa, Vietnam, stated that $61 \%$ of cases came from areas with a population density below 200 people within $100 \mathrm{~m}$ (6,360 people $\left./ \mathrm{km}^{2}\right)$, while $75 \%$ came from areas below 400 people within $100 \mathrm{~m}$ (12,730 people/ $\mathrm{km}^{2}$ ) (Yanai et al., 2007). Similarly, a report from Subang Jaya in Malaysia concluded that in an urban area with flats, apartments and condominiums, the population density per square meter is high, so dengue transmission can and will occur rapidly (Hsueh et al., 2012).

Our study does have some limitations. One would be an underestimation of the real incidence of dengue fever. In Hanoi city, each commune health station reports its dengue surveillance data to the district, which transmits it monthly to the Hanoi Preventive Medicine Center. However, it is known that not all dengue patients are treated and some are treated in private health services whose records are not included in the state system. As in many dengue-epidemic settings, the dengue surveillance system in Vietnam relies on passive reporting of clinically-diagnosed dengue in hospitalized patients. Less severe infections will probably not reach hospitals. Therefore, the real number of dengue infections will be much higher than the reported number (Cuong et al., 2011). The assumption that the numbers of clinically reported cases reflect the numbers of unreported cases in the same area is difficult to validate. The second restriction in our study is that we could not obtain high resolution maps for population density, so that the exact contribution of population density to the number of cases detected could not be included. Also complete road maps were not available, which reduces the accuracy of our predictions about the exact effect of these factors on dengue fever.

\subsection{Conclusions}

In summary, the results of our study in the urban setting of Hanoi show that population density, water surface area and road length are risk factors for dengue fever. We found that in the areas with higher population density, higher water surface ratio and higher street length ratio, there was a higher rate of dengue incidence. This information is very useful to target control activities especially in periods of high dengue transmission. Preventive medicine services should focus their efforts on populations near roads and water bodies, where 


\section{ENVIRONMENTAL FACTORS INFLUENCING EPIDEMICS OF DENGUE FEVER IN VIETNAM}

population density is also higher. When more detailed accurate data becomes available, the mapping of high risk areas that should have priority for control measures will also be more accurate. 


\section{Chapter 7}

\section{Hot spot detection and}

\section{spatiotemporal dispersion of}

\section{dengue fever in Hanoi,}

\section{Vietnam}

This chapter has been published as: Toan DTT, Hu W, Thai PQ, Hoat LN, Wright $P$, Martens $P$ (2013). Hot spot detection and spatiotemporal dispersion of dengue fever in Hanoi, Vietnam. Global Health Action, Vol 6 (2013). 


\section{HOT SPOT DETECTION AND SPATIOTEMPORAL DISPERSION OF DENGUE FEVER IN HANOI, VIETNAM}

\subsection{Introduction}

This chapter will visualize the spatio-temporal distributions of notified DF infection in Hanoi. Since the risk of dengue fever varies with space and time, it is necessity for a precise knowledge of the risk factors, the localities at risk, the level of risk, and details on the exposed population. In recent years, the development of geographic information systems (GIS) has provided spatial analytical information that has enabled epidemiologists to add a spatial component to traditional epidemiologic studies (Lawson and Williams, 2001). In the field of infectious and vector-borne diseases such as malaria or dengue fever, GIS have been widely used for mapping diseases of different pathologies, supporting analysis of the space and space-time distributions of disease data, to identify risk factors and map risk areas $(\mathrm{Hu}$ et al., 2011; Nguyen et al., 2011).

More recently, temporal, spatial, and space-time scan statistics (SaTScan) have been widely used to test whether clusters can be detected or if the point process is purely randomly distributed. The advantages of using SaTScan are that it can adjust for confounding variables, resolve pre-selection bias as it searches for clusters without specifying their size or location, provides a single p-value as the likelihood-ratio-based test takes account of multiple testing, and finally, it can be applied to a whole region to detect significant clusters in that region (Kulldorff, 2006).

We have investigated the spatial distribution of confirmed cases of DF and particular looked for areas of high risk within 14 districts of Hanoi using spatial scan statistics and GIS. In addition, we have used these tools to detect hot spots and identify the dynamics of the dispersion of DF over the time period between 2004 and 2009 in Hanoi.

\subsection{Method}

\subsubsection{Some features of Hanoi}

Hanoi is located in the north of country in the low lying and densely populated Red River delta. In 2009, the city had a population of about 6.5 million; the population density was quite high at 1943 people $/ \mathrm{km}^{2}$. The city is divided into 229 postcode areas whose 


\section{HOT SPOT DETECTION AND SPATIOTEMPORAL DISPERSION OF DENGUE FEVER IN HANOI, VIETNAM}

populations ranged in size from 3,027 to 35,302 (Figure 7.1 )
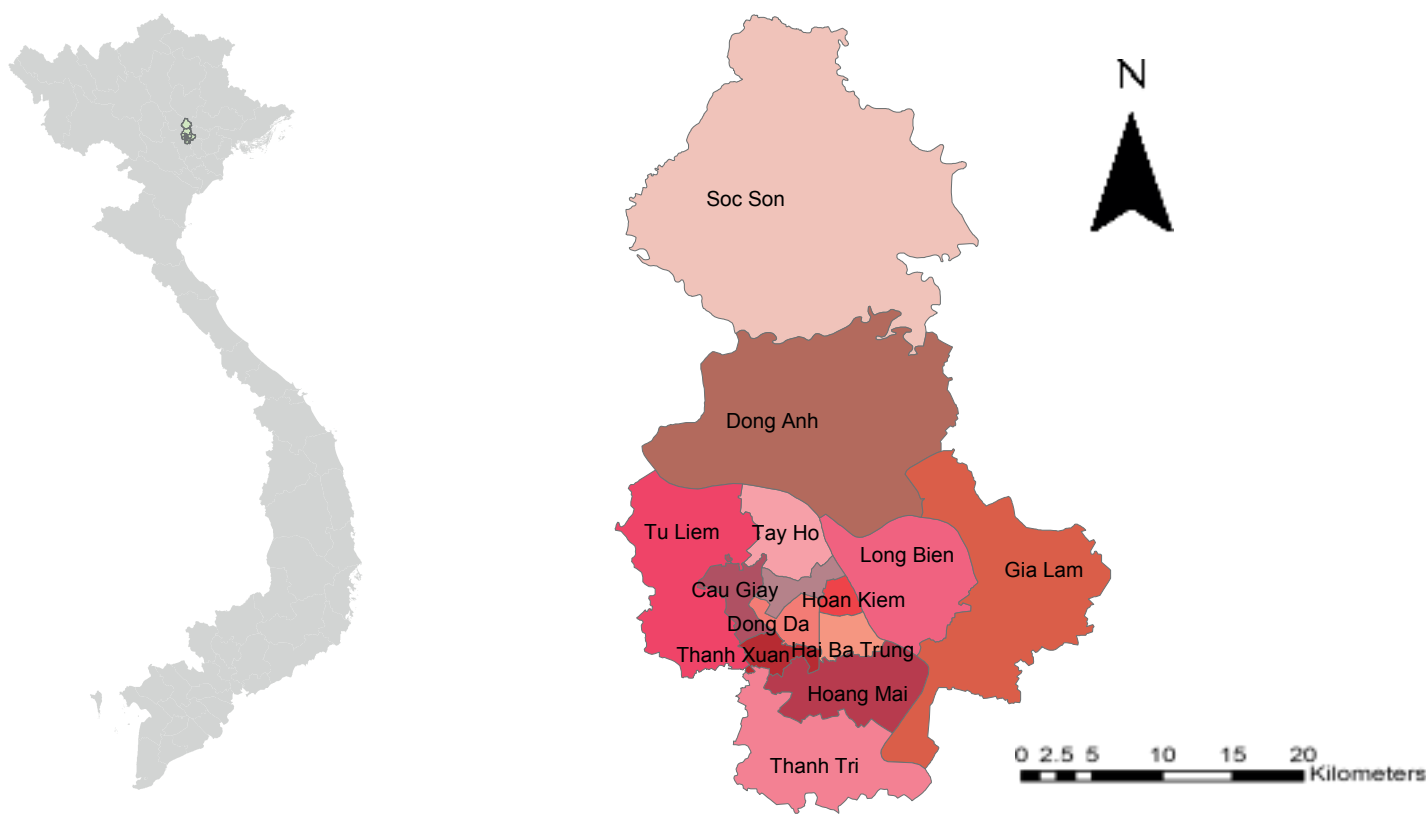

Figure 7.1: Location of the study area Hanoi, Vietnam.

Hanoi includes several rivers and lakes, many of which are seriously polluted due to rapid urban development with accompanying insufficiently processed municipal and industrial wastes. The rivers and lakes in the inner city create an environment for the development of many types of pathogenic microorganisms. Hanoi experiences the typical climate of northern Vietnam, where summers are hot and humid but winters are relatively cool and dry. The summer months from May to September receive the majority of the annual rainfall $(1,682$ mm rainfall/ year). The winter months from November to March are relatively dry, although spring often brings light rains. The minimum winter temperature in Hanoi can dip to as low as $6-7{ }^{\circ} \mathrm{C}\left(43-45^{\circ} \mathrm{F}\right)$ not including the wind chill, while summer can get as hot as $38-40$ ${ }^{\circ} \mathrm{C}\left(100-104^{\circ} \mathrm{F}\right)$. The period from May to September is also suitable for the development of mosquitos. The above characteristics make Hanoi a very favorable site for the existence, 


\section{HOT SPOT DETECTION AND SPATIOTEMPORAL DISPERSION OF DENGUE FEVER IN HANOI, VIETNAM}

circulation and development of dengue fever and dengue hemorrhagic fever.

\subsubsection{Data analysis}

Descriptive analysis Descriptive statistics of notified DF cases in Hanoi in three periods (2004-2005, 2006-2007, and 2008-2009), incidence rate of DF in these periods or choropleth maps were analyzed to visualise the dynamics of the disease. The monthly distribution of DF according to seasonality was also visualised using box plots.

Spatial autocorrelation analysis The spatial autocorrelation of the expected incidence rates of dengue fever in three different periods was assessed using Morals I statistic in ArcGIS 9.2. Spatial autocorrelation was considered significant if the $\mathrm{p}$-value $<0.05$. Morans I ranges from -1 to 1 and can be interpreted as follows: a value close to 0 indicates spatial randomness, while a positive value indicates positive spatial autocorrelation and a negative value indicates negative spatial autocorrelation.

Cluster analysis Scan statistics were used to detect and evaluate the clusters of cases in either a purely temporal, purely spatial or combined space-time setting. This was done by gradually scanning a window across time and/or space, noting the number of observed and expected observations inside the window at each location. For each location and each size of the scanning window, the alternative hypothesis was that there was an elevated risk within the window as compared to outside.

In SaTScan software, the scanning window was an interval (in time), a circle or an ellipse (in space) or a cylinder with a circular or elliptic base (in space-time). Multiple window sizes were used. The window with the maximum likelihood was the most likely cluster, that is, the cluster least likely to be due to chance. A p-value was assigned to that cluster. The standard purely spatial scan statistic imposed a circular window on the map. The window was in turn centered on each of several possible grid points positioned throughout the study region. For each grid point, the radius of the window varied continuously in size from zero to an upper limit specified by the user. In this way, the circular window was flexible both in its location and size, while each circle was a candidate cluster. The spacetime scan statistic was defined by a cylindrical window with a circular (or elliptic) geographic base and with 


\section{HOT SPOT DETECTION AND SPATIOTEMPORAL DISPERSION OF DENGUE FEVER IN HANOI, VIETNAM}

its height corresponding to the time. The base was defined exactly as for the purely spatial scan statistic, while the height reflected the time period of potential clusters. The cylindrical window was then moved in space and time, so that for each possible geographical location and size, it also visited each possible time period. In effect, an infinite number of overlapping cylinders of different sizes and shapes were obtained, which jointly cover the entire area studied and where each cylinder reflected a possible cluster. The temporal scan statistic used a window that moved in one dimension, time, defined in the same way as the height of the cylinder used by the space-time scan statistic. This meant that it was flexible in both start and end dates. For purely spatial and space-time analyses, SaTScan also identified secondary clusters in the data set in addition to the most likely cluster, and lined them up by their likelihood ratio test statistic. For purely temporal analyses, only the most likely cluster was reported. No geographic overlap was used as a default setting, so that secondary clusters would not overlap the most significant cluster. In order to scan from small to large clusters, the maximum cluster size was set to $50 \%$ of the total population at risk. To ensure sufficient statistical power, the number of Monte Carlo replications was set to 999 .

Dynamic dispersion of dengue fever

In this study, we tried to identify whether changes in DF varied with latitude and longitude of village centroids in the three periods. Logistic regression models can be constructed with the dichotomous outcome variable defined as whether or not an increase of DF occurred in each village between the three periods. Longitude and latitude of village centroids were entered as explanatory variables. Spatial dispersions can be expressed in terms of odds ratios (OR) for longitude and latitude, with 95\% confidence intervals (CI).

\subsection{Results}

\subsubsection{Descriptive analysis}

The monthly average numbers of postcode areas in Hanoi that had notified dengue cases for three periods: 2004-2005, 2006-2007, and 2008-2009, are summarized in Table 7.1. It was revealed that there was a clear trend of geographic expansion of dengue transmission in 


\section{HOT SPOT DETECTION AND SPATIOTEMPORAL DISPERSION OF DENGUE FEVER IN HANOI, VIETNAM}

Hanoi through these periods (with average numbers of postcode areas equal to 41.0, 49.33, and 79.33 respectively).

Table 7.1: Descriptive statistics of monthly numbers of postcode areas with notified dengue cases.

\begin{tabular}{lccccccc}
\hline Period & Mean & S.D. & Minimum & Q1* & Median & Q3† & Maximum \\
\hline $2004-2005$ & 41.00 & 32.36 & 5 & 15.75 & 36 & 59 & 95 \\
$2006-2007$ & 49.33 & 32.47 & 8 & 24.25 & 47 & 72.25 & 104 \\
$2008-2009$ & 79.33 & 45.53 & 17 & 36.75 & 74 & 122.5 & 146 \\
\hline
\end{tabular}

* Q1, first quartile value

+ Q3, third quartile value

Figure 7.2 presents a striking variation in monthly numbers of dengue cases and monthly numbers of postcode areas reporting dengue cases from 2004 to 2009. A large peak of dengue incidence occurred in October and November 2009 (3696 cases and 2698 cases). Peaks in incident cases coincided with high monthly numbers of postcode areas reporting dengue fever cases.

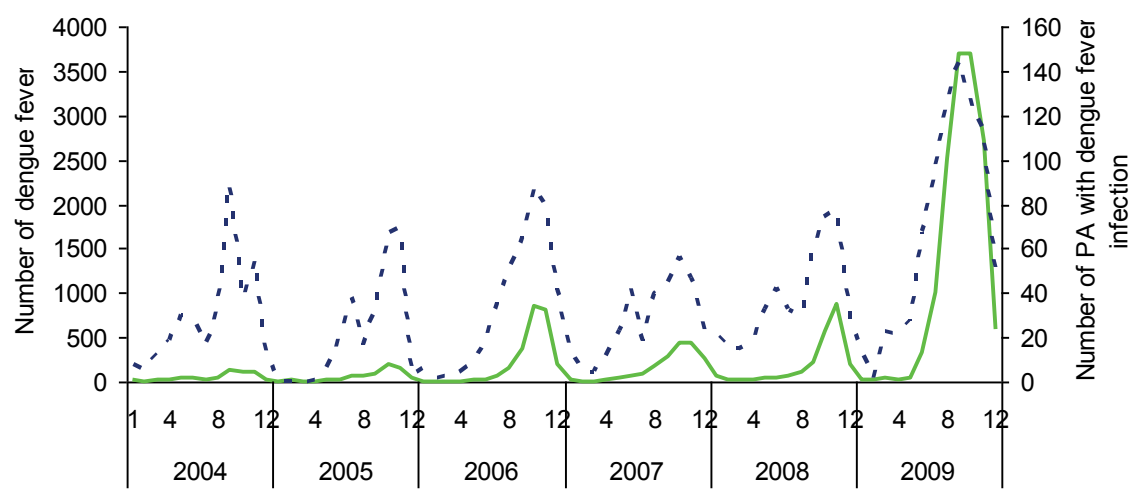

Figure 7.2: Numbers of dengue cases and postcode areas with dengue notifications between January 2004 and December 2009 in Hanoi. 


\section{HOT SPOT DETECTION AND SPATIOTEMPORAL DISPERSION OF DENGUE FEVER IN HANOI, VIETNAM}

Boxplots of the monthly numbers of postcode areas reporting dengue cases are shown in Figure 7.3. The visualization indicated a strongly seasonal pattern (with a peak in autumn and spring) and also suggested that there was an upward trend in dengue incidence from 2004 to 2009.

Winter

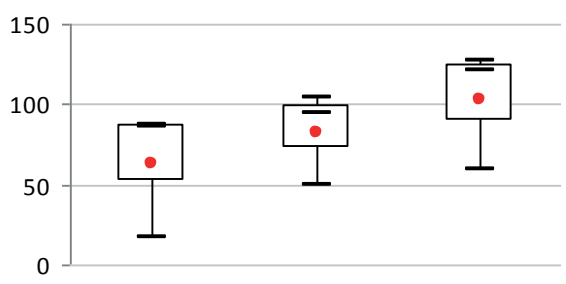

Autumn

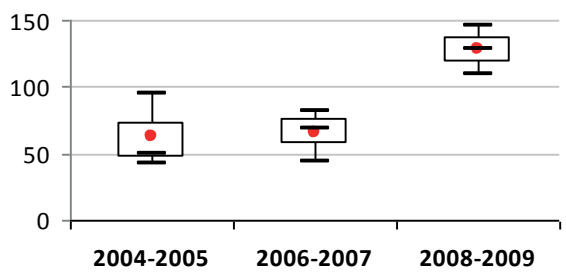

Spring

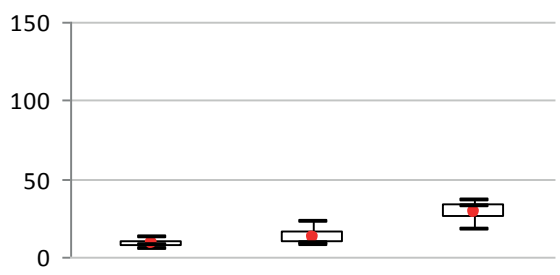

Summer

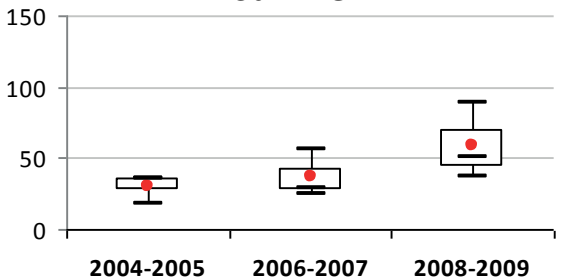

Figure 7.3: Boxplots of the seasonal distribution of numbers of postcode areas with dengue infection in three periods in Hanoi.

The boxplots display the values of the 25 th, 50 th and 75 th percentiles.

Figure 7.4 shows the geographic distribution of raw incidence of notified dengue cases in Hanoi during three time periods. There was an expansion of postcode areas with dengue to the north-western wards of Hanoi between 2004 and 2009. Dengue incidence ranged from 4.55 to 2,887.6 cases/100,000 population. The number of postcode areas reporting dengue cases kept increasing from 97 to 132 and then to 160 during the three periods.

\subsubsection{Spatial autocorrelation of dengue fever}

A significant positive spatial autocorrelation of dengue incidence for all three periods was shown in Table 7.2. Morans I index was 0.19 (expected Morans I = -0.01042, p-value $<0.001$ ) during 2004-2005 (expected Morans I = -0.00725, p-value =0.01), 0.32 during 2006- 


\section{HOT SPOT DETECTION AND SPATIOTEMPORAL DISPERSION OF DENGUE FEVER IN HANOI, VIETNAM}

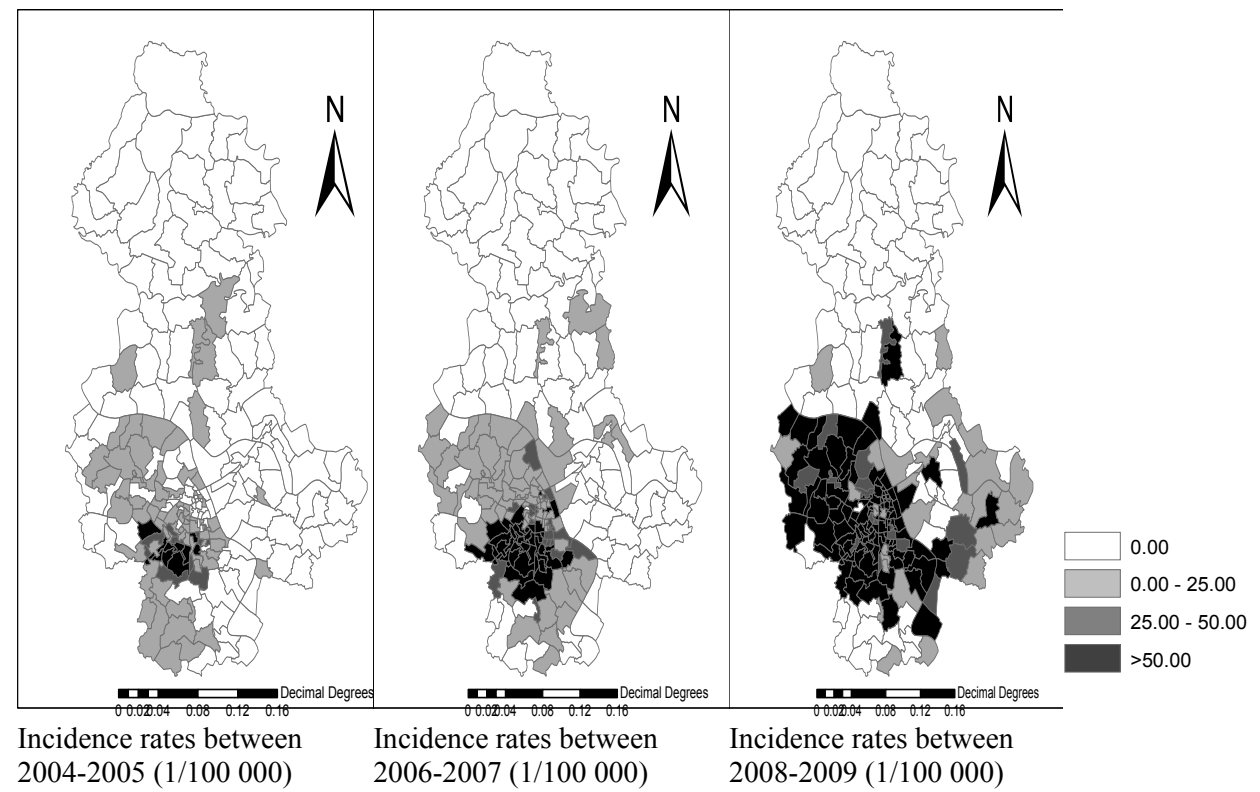

Figure 7.4: Map showing raw dengue incidence rates in three periods.

2007, and 0.22 (expected Morans I = -0.00629, p-value $=0.01$ ) during 2008-2009. This means that villages closer together tended to have more similar baseline incidence rates than those further apart.

Table 7.2: Spatial autocorrelation analysis for dengue in Hanoi, 2004-2009.

\begin{tabular}{llccc}
\hline Period & $\begin{array}{l}\text { Annual } \\
\text { Incidence }\end{array}$ & Moran's I & $E(I)$ & $p$ \\
\hline $2004-2005$ & 226.32 & 0.19 & -0.011 & 0.001 \\
$2006-2007$ & 1049.78 & 0.32 & -0.007 & 0.001 \\
$2008-2009$ & 3774.49 & 0.22 & -0.006 & 0.001 \\
\hline
\end{tabular}

\subsubsection{Purely temporal clustering}

The results of the purely temporal clustering analysis in each year could were consistent with the seasonal tendency of dengue transmission. Table 7.3 shows the temporal clusters of dengue cases in the study area from 2003 to 2009, which often included six months (June 


\section{HOT SPOT DETECTION AND SPATIOTEMPORAL DISPERSION OF DENGUE FEVER IN HANOI, VIETNAM}

to December)

Table 7.3: The clusters of dengue cases detected using the purely temporal analysis.

\begin{tabular}{|c|c|c|c|c|c|c|c|}
\hline Year & Cluster time frame & $\begin{array}{l}\text { Total days } \\
\text { of cluster }\end{array}$ & Obs $^{a}$ & $\operatorname{Exp}^{b}$ & Relative risk & $\operatorname{LLR}^{c}$ & p \\
\hline 2004 & $4 / 8-1 / 12$ & 118 & 216 & 160.30 & 1.90 & 17.68 & 0.001 \\
\hline 2005 & $5 / 6-1 / 12$ & 177 & 246 & 167.56 & 14.26 & 74.0 & 0.001 \\
\hline 2006 & $5 / 6-1 / 12$ & 177 & 1679 & 1556.38 & 2.30 & 45.39 & 0.001 \\
\hline 2007 & $5 / 7-31 / 12$ & 177 & 1051 & 937.56 & 1.88 & 33.36 & 0.001 \\
\hline 2008 & $4 / 8-16 / 12$ & 133 & 992 & 886.41 & 1.60 & 23.84 & 0.001 \\
\hline 2009 & $20 / 6-16 / 12$ & 179 & 7725 & 7634.84 & 1.34 & 12.64 & 0.001 \\
\hline
\end{tabular}

a: The number of observed cases in a cluster;

b: The number of expected cases in a cluster;

c: Log likelihood ratio

\subsubsection{Purely spatial clustering}

Analysis of purely spatial clustering of dengue cases from 2003 to 2009, with the maximum spatial cluster size set at $50 \%$ of the total population, identified the most likely cluster for each of the six years. However, only in 2003 and 2005 did the results show a random distribution of dengue in the space of Dong Da, Hai Ba Trung, Thanh Xuan and Hoang Mai districts (Table 7.4). For the years from 2006 to 2009, the risk was found to be the same inside and outside clusters, since the p-value $>0.05$.

Table 7.4: The clusters of dengue cases detected using the purely spatial analysis.

\begin{tabular}{cclccccc}
\hline Year & & Location & Obs $^{\mathbf{a}}$ & Exp $^{\mathbf{b}}$ & Relative risk & LLR $^{\mathbf{c}}$ & $\mathbf{p}^{\text {P }}$ \\
& & & & & & & \\
\hline 2004 & A & Thanh Tri & 175 & 150.08 & 1.33 & 3.56 & 0.932 \\
2005 & A & Hai Ba Trung, Hoang Mai & 65 & 37.10 & 2.01 & 10.4 & 0.002 \\
& B & Thanh Xuan, Dong Da & 128 & 96.19 & 1.66 & 8.18 & 0.017 \\
2006 & A & Dong Da, Tay Ho, Long Bien & 833 & 806.05 & 1.06 & 0.81 & 1.000 \\
2007 & A & Dong Da, Ba Dinh & 658 & 609.28 & 1.17 & 3.89 & 1.000 \\
2008 & A & Tu Liem, Cau Giay & 238 & 215.30 & 1.13 & 1.41 & 1.000 \\
2009 & A & Hoan Kiem, Hai Ba Trung & 2346 & 2323.1 & 1.02 & 0.16 & 1.000 \\
\hline
\end{tabular}

a: The number of observed cases in a cluster; b: The number of expected cases in a cluster; c: Log likelihood ratio

A: Most likely cluster; B: Secondary cluster 


\section{HOT SPOT DETECTION AND SPATIOTEMPORAL DISPERSION OF DENGUE FEVER IN HANOI, VIETNAM}

\subsubsection{Space-Time Clustering}

The space-time clustering analysis of the dengue data from 2004 to 2009 was also performed. Figure 7.5 and Table 7.5 present the clusters in all districts of Hanoi at a 5\% significance level $(\mathrm{p}<0.05)$ in this period.

Table 7.5: SaTScan statistics for space-time clusters with significantly higher incidence in Hanoi from 2004-2009 (Most likely cluster).

\begin{tabular}{lclcc}
\hline Location & Radius $(\mathbf{k m})$ & Time frame & Relative Risk & p-value \\
\hline Hoang Mai & 2.50 & $4 / 8-19 / 7 / 2004$ & 2.03 & 0.001 \\
Thanh Xuan & 0.68 & $17 / 11-31 / 12 / 2005$ & 2.66 & 0.001 \\
Dong Da & 0.64 & $2-16 / 11 / 2006$ & 3.51 & 0.001 \\
Dong Da & 0.99 & $17 / 11-16 / 12 / 2007$ & 3.38 & 0.001 \\
Hoang Mai & 0.29 & $19 / 8-2 / 10 / 2008$ & 5.58 & 0.001 \\
Hoan Kiem, Hai Ba & 1.56 & $2-16 / 11 / 2009$ & 6.41 & 0.001 \\
Trung & & & & \\
\hline
\end{tabular}

The results reveal a highly significant space-time association for dengue fever transmission. Six for the fourteen districts of Hanoi had significant cluster patterns, among which Dong Da, Hoang Mai and Thanh Xuan had the highest number of space-time clusters. The most likely cluster was found to differ across the time periods. In 2004-2005, only a few clusters were found, distributed in a large distance and time. In 2006, 2007 the most likely cluster occurred in Dong Da, with 149 cases and within 14 days of November. In August, 2008, the most likely cluster was reported in Hoang Mai and limited within 300m (RR = $5.58, \mathrm{p}=0.01$ ). In November, 2009, the highest number of dengue cases, 553, was again found in Dong Da and Hoan Kiem districts, within a radius of $1500 \mathrm{~m}$. The RR within the most likely cluster was $6.41(\mathrm{p}=0.001)$. The secondary clusters reported in Hoang Mai, Tay Ho and Hai Ba Trung were also limited at $1000 \mathrm{~m}$ and within 29 days.

\subsubsection{Dynamic dispersion of DF}

Logistic regression models were constructed to identify whether changes in DF varied with latitude and longitude of postcode centroids in the three periods, as presented in Table 7.6 .

The results indicated that changes of dengue fever were significantly associated with 

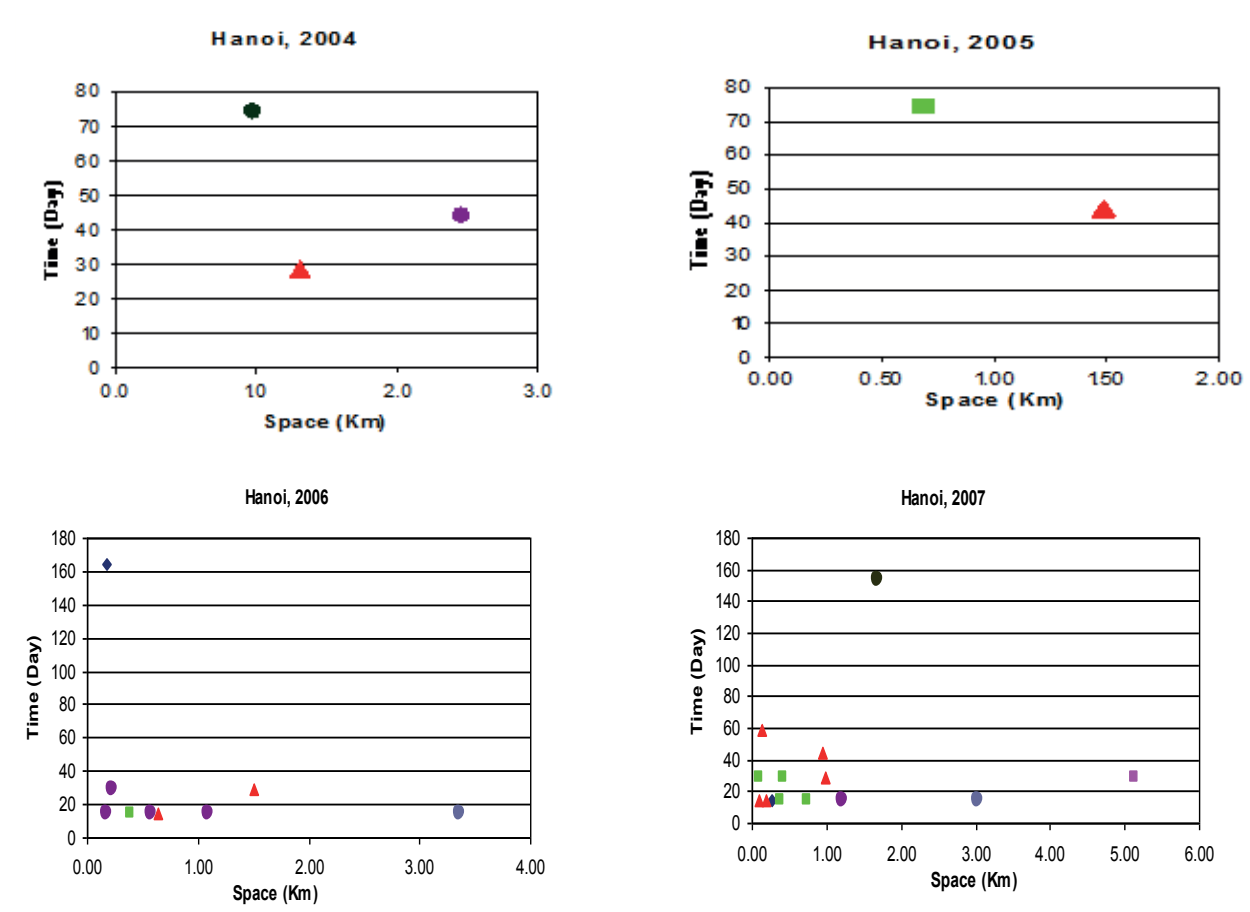

$\bullet$ Hoan Kiem • Hai Ba Trung $•$ Thanh Xuan $\bullet$ Dong Da • Hoang Mai • Tu Liem • Ba Dinh
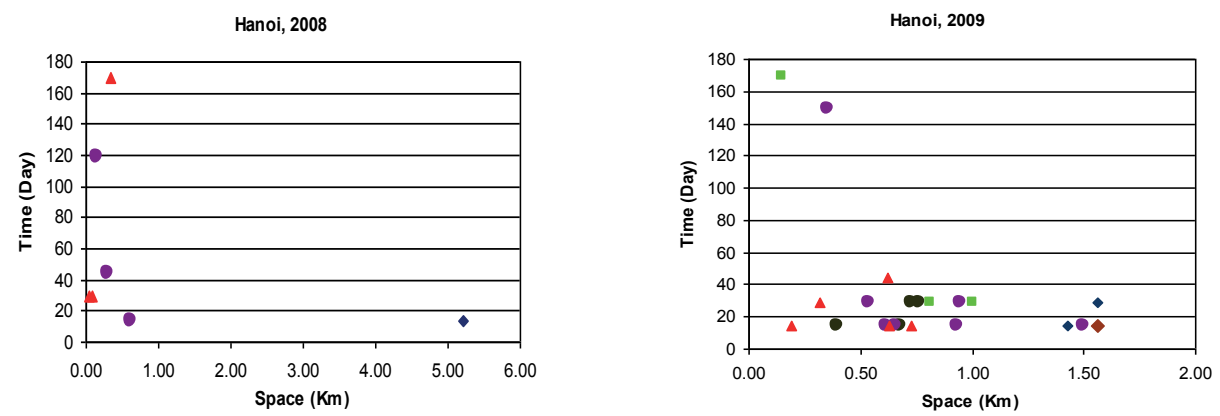

Figure 7.5: Scatterplot of significant space-time clusters in Hanoi from 20042009. 


\section{HOT SPOT DETECTION AND SPATIOTEMPORAL DISPERSION OF DENGUE FEVER IN HANOI, VIETNAM}

\begin{tabular}{lcccc}
\hline & \multicolumn{2}{c}{ Latitude } & \multicolumn{2}{c}{ Longtitude } \\
\cline { 2 - 5 } Change in periods & OR & $\mathbf{9 5 \% ~ C l}$ & OR & $\mathbf{9 5 \% ~ C l}$ \\
\hline Period 3 - Period 1 & 1.17 & $1.02-1.34$ & 1.06 & $0.93-1.34$ \\
Period 2 - Period 1 & 0.94 & $0.85-1.03$ & 0.98 & $0.87-1.11$ \\
Period 3 - Period 2 & 1.09 & $0.99-1.22$ & 0.98 & $0.89-1.06$ \\
\hline
\end{tabular}

* Period 1: 2004-2005; Period 2: 2006-2007; Period 3: 2008-2009

Table 7.6: Changes of dengue fever in latitude and longitude, Hanoi, 2004-2009.

latitude between the periods 2008-2009 (OR 1.17, 95\% CI 1.02-1.34) and 2004-2005 (OR $1.0695 \%$ CI 0.93-1.34), and associated with latitude in 2006-2007 (OR 0.94, 95\% CI 0.851.03) and longitude (OR 0.98, 95\% CI 0.87-1.11)

\subsection{Discussion}

Previous studies have shown that, in Vietnam, dengue epidemics often had a cycle of 3-5 years and were expected to reach 10-year peaks (Cuong et al., 2011; Nguyen et al., 2011; Pham et al., 2011). These peaks were observed in 1987, 1998 and most recently 2009, as also recorded in this study with an upward trend of dengue epidemics and the largest outbreaks in 2009. Most of the dengue cases occurred between June and November, when the rainfall and temperature were at their highest for the year. This time period was again observed in our purely temporal clustering, which revealed high-risk months in each year between 2004 and 2009. These results are consistent with those conducted in a Central Highlands province of Vietnam that found dengue to be most prevalent in the wet season (Pham et al., 2011). As in other tropical countries, Vietnams weather is favorable for the transmission of dengue fever. A warm temperature is crucial to the mosquitos life and gonotrophic cycle, and to virus replication. Sources of stagnant water and higher humidity could augment the epidemic during rainy season (Cazelles et al., 2005; Martens, 2002; Martens and Huynen, 2001; Moore et al., 2005).

Results from space-time clustering identified the high-risk areas over the larger region and through the years. Using the maximum spatial cluster size of $50 \%$ of the total population, and the maximum temporal cluster size of $50 \%$ of the total population, we identified six districts among the fourteen in Hanoi as having a significant cluster pattern within periods 


\section{HOT SPOT DETECTION AND SPATIOTEMPORAL DISPERSION OF DENGUE FEVER IN HANOI, VIETNAM}

of 29 days and limited to $1000 \mathrm{~m}$ on average. The areas that recorded the highest number of space-time clusters were Dong Da, Hoang Mai and Thanh Xuan with expansion to the northwestern wards of Hanoi, where a higher population is concentrated. A study in Malaysia revealed similar results, when a geographical weighted regression analysis suggested that the spatial distribution of dengue fever was closely related to population distribution (Moore et al., 2005). Moreover, their result from space-time permutation scan statistics showed that most of the clusters detected were located in areas of medium or high population density. Indeed, spatial clustering of disease is almost inevitable because human populations generally live in spatial clusters rather than in a random distribution through space.

Finally, we found spatial autocorrelation and logistic regression analysis to be valuable tools to study how spatial patterns change over time (Barbazan et al., 2010; Lu et al., 2009; Nishiura, 2006; Ooi and Gubler, 2009; Wu et al., 2007). In this study, we found that dengue fever had high spatial autocorrelation in three different time periods. It was closely related to the topography of the environment, and villages that were closer together tended to have more similar baseline incidence rates.

To the best of our knowledge, this is the first study to apply a spatial scan technique, using SaTScan, to investigate the temporal and geographical clustering of dengue fever in Vietnam, certainly in Hanoi. The study produced useful information on the prevailing epidemiological situation of dengue fever in Hanoi city by identifying hot spots of DF. The new knowledge about the presence of such hotspots in the city can help the Hanoi Preventive Medicine Center to intensify remedial measures in the identified areas of high DF prevalence and to chalk out future strategies for more effective DF control.

This study does have potential limitations. The data on diseases were not obtained through a survey study but through sentinel surveillance, as only patients presenting themselves in hospitals were taken into account. The study analysed the statistically significant clusters of DF in Hanoi but did not examine their causes. Future research should focus on the effects of various socio-economic and environmental factors which could affect the transmission patterns of dengue fever.

In summary, this study has demonstrated the presence of long term hotspots of DF in the city of Hanoi, which were the districts of Dong Da, Hoang Mai and Thanh Xuan. 


\section{HOT SPOT DETECTION AND SPATIOTEMPORAL DISPERSION OF DENGUE FEVER IN HANOI, VIETNAM}

It also showed an expansion of geographic distribution of dengue fever over recent years. Based on these findings, it is necessary to further improve our understanding of the impact of socio-environmental change and ecosystem stress on the transmission of dengue fever. The study has also demonstrated that using existing health data, the spatial scan statistic and GIS can provide public health officials with necessary evidence about the prevalence of statistically significant hotspots of DF in the city, thus enabling them to chalk out more effective strategies to contain the epidemics. 


\section{Chapter 8}

\section{Perceptions on climate}

\section{change and its impact on}

\section{human health}

This chapter has been published as: Toan DTT, Kien VD, Giang KB, Minh HV, Wright $P$ (2014). Perceptions on climate change and its impact on human health: an integrating quantitative and qualitative approach. Glob Health Action. 2014; 7: 10.3402/gha.v7.23025. 


\section{PERCEPTIONS ON CLIMATE CHANGE AND ITS IMPACT ON HUMAN HEALTH}

\subsection{Introduction}

Climate change is one of the most important environmental changes population will face in the coming decades. Understanding how it may affect human health and disease is complex and requires a thorough understanding of links between present climate and diseases (Epstein, 2005). However, in a study conducted recently by Gallup Poll in 128 countries, still one third of residents from Africa, parts of Asia and the Middle East, and a few countries from the former Soviet Union still not recognised climate change as a result of human activities (Dunlap and McCright, 2008).

The link between population health and climate change has been demonstrated by scientists who stated that climate change poses a wide range of risks to population health. As noted by the World Health Organization, climate change is not just a threat to biological systems and the environment but a significant and emerging threat to public health, especially in lower income populations and tropical/subtropical countries (Be et al., 2007). Protecting health from the impacts of climate change is recognized as one of the defining challenges in this century (WHO, 2009). Perceptions on effect of climate change and health have also already been studied among community members in some other developed (Dolan and Walker, 2006; Gonzalez and da Silveira, 1997; Leiserowitz, 2005; McMichael and Butler, 2006; Preet et al., 2010) and developing countries (Carew-Reid, 2008; Chaudhary and Bawa, 2011; Emch et al., 2008; Haque et al., 2012). Haque et al. (2012) conducted a study in Bangladesh and found that most local perceptions on climate change were consistent with the scientific evidence regarding the vulnerability of Bangladesh to climate change. They perceived that the changes in heat, cold and rainfall had occurred over the last five to ten years. They also linked these problems to identify future threats to themselves, families and livelihoods.

Understanding the complex links between climate change and human health as well as understanding peoples concerns will be the first step to assist policy makers to develop communication strategies to engage communities in each location most effectively. Such communication is increasingly a priority for Hanoi City, because in recent years, climate change has affected the city with extreme consequences (NTP, 2008). The results of this 


\section{PERCEPTIONS ON CLIMATE CHANGE AND ITS IMPACT ON HUMAN HEALTH}

study could be used to inform decision makers in Hanoi responsible for developing or adapting programs of action to cope with climate change.

\subsection{Methods}

\subsubsection{Study setting}

Hanoi, the capital of Vietnam, is comprised of 29 districts, 10 of which are urban and 19 rural. As of 2009, Hanois population was estimated at 6.5 million, of which 2.6 million (41\%) lived in urban districts (GSO, 2012). The study was carried out in four urban districts in Hanoi, namely Ba Dinh, Hoan Kiem, Hai Ba Trung and Dong Da districts, selected because they included a number of slum areas. We defined the slum areas according to the United Nations definition, as areas where people live in temporary houses, insecure locations, and narrow spaces or polluted environmental conditions.

\subsubsection{Study design and participants}

This was a population-based cross-sectional study conducted from December 2012 to March 2013, applying a mixed-methods research design, quantitative and qualitative (Côte et al., 1993; Patton, 2002). To obtain a wider range of household perceptions, a quantitative survey was conducted in 30 slum areas (randomly selected from the list of 84 slum areas) and 30 matched, nearby non-slum areas in the four urban districts. In each slum or non-slum area, about 20 households were selected; we applied a door-to-door approach after randomly selecting the first house. In each household, we selected one individual among the household members between 15 and 60 years of age by chance, and a second individual above 60 years old, if available. The non-response rate was about 2-3\%, and quite similar in both slum and non-slum areas. Finally 1,444 people were invited to participate, including 754 people in non-slum areas and 690 people in slum areas. For the qualitative part, to gain insights into local perceptions and experiences of people in Hanoi, two focus group discussions and twelve key informant interviews were conducted. The participants were selected purposively from both slum and non-slum areas in Hanoi. They included 16 community members, 4 local 


\section{PERCEPTIONS ON CLIMATE CHANGE AND ITS IMPACT ON HUMAN HEALTH}

political leaders and 4 district health staff.

\subsubsection{Data collection}

In the quantitative study, face-to-face interviews using structured questionnaires were conducted by trained medical students from Hanoi Medical University. The interviewers were supervised by senior staff of the Center for Health System Research, Hanoi Medical University. The questionnaires were developed by the research team and were piloted before used in the field. The questions included topics such as perception of climate change, impact of climate change, changes in climate as well as illness of family members in summer and winter compared to five years earlier. To characterize the socioeconomic status of the study population, we used principal components analysis to calculate a wealth index based on different variables, including: ownership of household, radio, computer, internet connection, telephone, refrigerator, etc. The wealth index was estimated separately for the non-slum and slum areas, then divided into five quintiles, ranking from the poorest to the richest.

An interview guide based on the broad themes of hot and cold weather and peoples perception of climate change was used for the qualitative study. Prior to the official study, a pilot interview was carried out with one female health staff of the slum area. Interviews were conducted at the interviewees house; focus group discussions were organized in the meeting rooms of two District Health Centers. All of the interviews were recorded both in notes and recordings, with the agreement of the interviewees, and later transcribed.

\subsubsection{Data analysis}

Data were analyzed using Stata statistical software, version 12.1. Both descriptive and analytical statistics were applied. The chi-square test was used to compare discrete variables. Logistic regressions were used to detect any association between peoples perceptions of climate change and the impacts of climate change and the social determinants. The independent variables (considered as social determinants) consisted of area of household (slum or non-slum area), education, age group and socioeconomic status. Significance level was set at $\mathrm{p}<0.05$. 


\section{PERCEPTIONS ON CLIMATE CHANGE AND ITS IMPACT ON HUMAN HEALTH}

Data for qualitative study was transcribed and described according to the above themes of perceptions of climate change and perceptions of impact of climate change on health. Content analysis was used to analyze the interview transcripts. Content analysis is a procedure that organizes transcribed material by coding interview data into blocks that represent a common theme or new themes that emerge from the interviewee quotes (Côte et al., 1993).

\subsubsection{Ethical considerations}

People invited to participate in the study were asked for their informed consent before they were interviewed. They were informed that they were free to withdraw from the study at any time.

\subsection{Results}

\section{The characteristics of the study subjects}

Of the 1444 people selected by the sampling procedure, $1412(97.8 \%)$ were available and agreed to participate and provided information.

Table 8.1 shows a description of the demographic characteristics of the study population according to living area (non-slum or slum area). The mean age of the respondents was $55.6 \pm 16.5$ years (range 16 to 92 ) in the non-slum areas and $51.3 \pm 16.5$ (range 16 to 95) in the slum areas. The distribution of gender, marital status and wealth index was quite similar between two areas. However, there was a significant difference in the distribution of age groups, education and household size between them. People in the non-slum area seemed to have higher education levels than those in the slum areas, especially more of them had college education.

\subsubsection{Perception of climate change}

Most of the survey respondents had heard about climate change and its impact (Table 8.2). The proportion of people having heard about climate change and its impact on people was significantly higher in the non-slum area. Among those who had heard about climate 


\section{PERCEPTIONS ON CLIMATE CHANGE AND ITS IMPACT ON HUMAN HEALTH}

Table 8.1: Demographic characteristics of study population ( $\mathrm{n}=1412$ people)

\begin{tabular}{|c|c|c|c|c|}
\hline & Non-slum area (\%) & Slum area (\%) & $x^{2}$ & $P$ \\
\hline \multicolumn{5}{|l|}{ Gender } \\
\hline Female & $482(65.8)$ & $436(64.2)$ & 0.42 & 0.52 \\
\hline Male & $251(34.2)$ & $243(35.8)$ & & \\
\hline \multicolumn{5}{|l|}{ Age } \\
\hline $15-24$ & $24(3.3)$ & $38(5.6)$ & 32.08 & 0.00 \\
\hline $25-34$ & $76(10.4)$ & $94(13.8)$ & & \\
\hline $35-44$ & $101(13.8)$ & $123(18.1)$ & & \\
\hline $45-54$ & $104(14.2)$ & $128(18.9)$ & & \\
\hline $55-64$ & $195(26.6)$ & $139(20.5)$ & & \\
\hline $65-74$ & $144(19.7)$ & $89(13.1)$ & & \\
\hline $75+$ & $89(12.1)$ & $68(10.0)$ & & \\
\hline \multicolumn{5}{|l|}{ Education } \\
\hline Primary school or less & $50(6.8)$ & $137(20.2)$ & 98.90 & 0.00 \\
\hline Secondary school & $158(21.6)$ & $199(29.3)$ & & \\
\hline High school & $209(28.5)$ & $187(27.5)$ & & \\
\hline College/University & $316(43.1)$ & $156(23.0)$ & & \\
\hline \multicolumn{5}{|l|}{ Marital status } \\
\hline Unmarried & $55(7.5)$ & $62(9.1)$ & 4.02 & 0.13 \\
\hline Married & $563(76.8)$ & $490(72.2)$ & & \\
\hline Divorced/Widowed & $115(15.7)$ & $127(18.7)$ & & \\
\hline \multicolumn{5}{|l|}{ Household size } \\
\hline 1-3 members & $227(31.0)$ & $329(48.5)$ & 46.21 & 0.00 \\
\hline 4-6 members & $458(62.5)$ & $309(45.5)$ & & \\
\hline$>=7$ members & $48(6.6)$ & $41(6.04)$ & & \\
\hline \multicolumn{5}{|c|}{ Socio-economic status (Wealth index) } \\
\hline $1^{\text {th }}$ (poorest) & $145(19.8)$ & $141(20.8)$ & 3.29 & 0.51 \\
\hline $2^{\text {th }}$ & $158(21.6)$ & $124(18.3)$ & & \\
\hline $3^{\text {th }}$ & $144(19.7)$ & $142(20.9)$ & & \\
\hline $4^{\text {th }}$ & $154(21.0)$ & $136(20.0)$ & & \\
\hline $5^{\text {th }}$ (richest) & $132(18.0)$ & $136(20.0)$ & & \\
\hline
\end{tabular}




\section{PERCEPTIONS ON CLIMATE CHANGE AND ITS IMPACT ON HUMAN HEALTH}

change in both slum and non-slum areas, two thirds were aware of the term climate change and interpreted it as storm, flood, deep cold and long heat wave. The impact of climate change most often mentioned by both groups was the impact on human health $(92.3 \%$ and 91.3\% for slum and non-slum areas). However, a small number of respondents were not aware of this term and mentioned other phenomena such as earthquakes or air pollution as manifestations of climate change ( $13.1 \%$ and $12.2 \%$ in non-slum and slum areas, respectively).

Table 8.2: Perceptions of climate change and its impact.

\begin{tabular}{|c|c|c|c|c|}
\hline & Non-slum area (\%) & Slum area (\%) & $x^{2}$ & $P$ \\
\hline $\begin{array}{l}\text { Have you ever heard about climate change? } \\
(n=1412)\end{array}$ & $581(79.3)$ & $476(70.1)$ & 15.72 & 0.00 \\
\hline \multicolumn{5}{|l|}{$\begin{array}{l}\text { If yes, what could be evidence of climate change? } \\
(n=1057)\end{array}$} \\
\hline Storm & $382(65.8)$ & $325(68.3)$ & 0.76 & 0.39 \\
\hline Flood & $380(65.4)$ & $306(64.3)$ & 0.14 & 0.71 \\
\hline Deep cold & $374(64.4)$ & $289(60.7)$ & 1.50 & 0.22 \\
\hline Drought & 274(47.2) & 231(48.5) & 0.20 & 0.66 \\
\hline Long heat wave & $394(67.8)$ & $302(63.5)$ & 2.22 & 0.14 \\
\hline $\begin{array}{l}\text { Other (tsunami, earthquake, pollution, hail } \\
\text { etc.) }\end{array}$ & $76(13.1)$ & $58(12.2)$ & 0.19 & 0.66 \\
\hline $\begin{array}{l}\text { Have you ever heard about the impact of climate } \\
\text { change? }(n=1367)\end{array}$ & $596(81.3)$ & $470(69.2)$ & 27.85 & 0.00 \\
\hline \multicolumn{5}{|l|}{$\begin{array}{l}\text { If yes, what might be visible impact of climate } \\
\text { change? }(n=1066)\end{array}$} \\
\hline Impact on health & $544(91.3)$ & $434(92.3)$ & 0.39 & 0.53 \\
\hline Impact on crops & $230(38.6)$ & $166(35.3)$ & 1.20 & 0.27 \\
\hline Impact on livestock & 202(33.9) & $147(31.3)$ & 0.82 & 0.36 \\
\hline Impact on environment & $448(75.2)$ & $324(68.9)$ & 5.11 & 0.02 \\
\hline Unknown & $14(2.4)$ & $8(1.7)$ & 0.54 & 0.46 \\
\hline
\end{tabular}

This incorrect perception was explained by one man, 39 years old: I heard from the television that the areas affected by earthquake are often highly vulnerability to climaterelated problems, for example more extreme storms Key informant interviews and focus group discussions revealed that most perceived that climate change is occurring in the form of changes in rainfall and temperature; one third perceived it as a change of strong wind during the rainy season. We also asked the participants in a focus group discussion to give their views on the main reason for the changes in climate. Two people said, It is caused mostly by human activities. while others mentioned that It is caused by both the natural 


\section{PERCEPTIONS ON CLIMATE CHANGE AND ITS IMPACT ON HUMAN HEALTH}

changes in the environment and human activities.

Table 8.3: Perceived changes in heat and cold compare to five years ago $(n=1412)$.

\begin{tabular}{|c|c|c|c|c|}
\hline & Non-slum area (\%) & Slum area (\%) & $x^{2}$ & $\mathrm{P}$ \\
\hline \multicolumn{5}{|c|}{$\begin{array}{l}\text { How do you perceive the temperature in the } \\
\text { last summer compared to summer five years } \\
\text { ago? }\end{array}$} \\
\hline Hotter & $514(70.1)$ & $464(68.3)$ & 9.48 & 0.02 \\
\hline No difference & $148(20.2)$ & $116(17.1)$ & & \\
\hline Cooler & $53(7.2)$ & $69(10.2)$ & & \\
\hline Unknown & $18(2.5)$ & $30(4.4)$ & & \\
\hline \multicolumn{5}{|c|}{$\begin{array}{l}\text { Do you perceive any change in illness among } \\
\text { family members during last summer } \\
\text { compared to summer five years ago? }\end{array}$} \\
\hline More illness & 254(34.6) & $260(38.3)$ & 3.22 & 0.36 \\
\hline No difference & $457(62.4)$ & $394(58.0)$ & & \\
\hline Less illness & $15(2.05)$ & $19(2.8)$ & & \\
\hline Unknown & $7(0.95)$ & $6(0.9)$ & & \\
\hline \multicolumn{5}{|c|}{$\begin{array}{l}\text { How do you perceive the temperature during } \\
\text { the last winter compared to winter five years } \\
\text { ago? }\end{array}$} \\
\hline Colder & $453(61.8)$ & $385(56.7)$ & 16.67 & 0.00 \\
\hline No difference & $156(21.3)$ & $127(18.7)$ & & \\
\hline Warmer & $87(11.9)$ & 134(19.7) & & \\
\hline Unknown & $37(5.1)$ & $33(4.9)$ & & \\
\hline \multicolumn{5}{|c|}{$\begin{array}{l}\text { Do you perceive any change in illness among } \\
\text { your family members during the last winter } \\
\text { compared to winter five years ago? }\end{array}$} \\
\hline More illness & 256(43.9) & $252(37.1)$ & 1.52 & 0.68 \\
\hline No difference & 447(61.0) & 402(59.2) & & \\
\hline Less illness & $21(2.9)$ & $20(2.95)$ & & \\
\hline Unknown & $9(1.2)$ & $5(0.74)$ & & \\
\hline
\end{tabular}

As shown in Table 8.3, the majority of the respondents in both non-slum and slum areas stated that changes in climate had occurred compared to five years ago. There were significant differences between those living in non-slum and slum areas in terms of their perception of climate variability. However, there were no significant differences between the non-slum and slum areas when respondents reported about changes in patterns of illness in their families. About one-third of respondents reported that illness among family members had increased during both summer and winter compared to five years earlier. Participants in the qualitative study were also asked to reflect on their perceptions of any changes in illness 


\section{PERCEPTIONS ON CLIMATE CHANGE AND ITS IMPACT ON HUMAN HEALTH}

among their family members compared to five years earlier. Most of those interviewed said that their family members, especially children, became ill more easily than some years in the past. One health staff said: The number of children coming to our health center has been increasing recently. The hot and humid environment in Hanoi is favorable for the development of bacteria, insects and other disease carriers such as flies and rats. Children are more vulnerable to these diseases. According to the respondents, the most common symptoms due to hot weather were headache, fatigue and dizziness, and hypertension and other cardiovascular diseases were common. The symptoms most often mentioned in relation to cold weather were cough and fever, and common diseases were pneumonia, influenza and emerging infectious diseases such as dengue fever or Japanese encephalitis. For example, a male house owner said, Nowadays, there is an increase of infectious diseases such as dengue fever. It occurred periodically throughout the year. The hot weather and rainfall have created favorable conditions for breeding of dengue mosquitoes.

\subsubsection{Perception of impacts of climate change and social de- terminants}

Table 8.4 presents the results of the logistic regression analysis of correlates of perception of climate changes impact and selected social determinants.

The analysis revealed that for those who lived in both slum and non-slum areas, the perception of climate change impact was significantly associated with gender and education. Men were almost 1.6 times more likely to have heard of impacts of climate change than were women. Respondents in non-slum areas who had completed high school or university were 5.8 times and 12.9 times more likely to have heard of climate change than those who had not completed primary school. The odds ratios were 4.9 times and 11.1 times for slum areas respectively. There was no significant association between the perception of impact of climate change and socio-economic status. 


\section{PERCEPTIONS ON CLIMATE CHANGE AND ITS IMPACT ON HUMAN HEALTH}

Table 8.4: Associations between perception of the impact of climate change and social determinants .

\begin{tabular}{lcccc}
\hline & Non-slum area $(\mathrm{n}=733)$ & \multicolumn{2}{c}{ Slum area $(\mathrm{n}=679)$} \\
\hline Independent variables & OR & $\mathrm{p}$ & OR & $\mathrm{p}$ \\
& & & & \\
\hline Gender & & & & \\
$\quad$ Male & 1.6 & 0.04 & 1.6 & 0.02 \\
$\quad$ Female & 1 & - & 1 & - \\
\hline Education & 1 & - & 1 & - \\
$\quad$ Primary School or below & 3.5 & 0.00 & 1.9 & 0.00 \\
\hline Secondary School & 5.8 & 0.00 & 4.9 & 0.00 \\
\hline High School & 12.9 & 0.00 & 11.1 & 0.00 \\
\hline College/University & & & & \\
\hline Socio-economic Status (Wealth Index) & 1 & - & 1 & - \\
\hline $1^{\text {th }}$ (poorest) & 1.2 & 0.42 & 2.7 & 0.00 \\
$2^{\text {th }}$ & 0.9 & 0.29 & 1.9 & 0.02 \\
$3^{\text {th }}$ & 1.0 & 0.31 & 2.7 & 0.00 \\
\hline $4^{\text {th }}$ & 0.6 & 0.17 & 3.4 & 0.00 \\
\hline $5^{\text {th }}$ (richest) & & & & \\
\hline
\end{tabular}

\subsection{Discussion}

Most of those living in non-slum areas and more than half living in slum areas had some perception of changes in climate and their effect on health. According to the majority of respondents, climate change is occurring and results in an increase of temperature during the summer and decrease of temperature during the winter, compared to five years ago. They also had the perception that storms, floods, deep cold and long heat waves were manifestations of climate change. Similar observations have been reported in various reports about climate change from both developed and developing countries including Japan, Laos, the Philippines, Bangladesh and several African countries (Aoyagi-Usui, 2008; Chaudhary and Bawa, 2011; Emch et al., 2008; Gonzalez and da Silveira, 1997; Leiserowitz, 2005). In Japan, in 2008, Aoyagi-Usui compared public opinion surveys from 1997, 2002, 2006 and 2007, and reported that peoples awareness of environmental issues was gradually becoming focused on global warming; people were increasingly worried that the rising average temperature of the world would have devastating effects on human life in the next century. This phenomenon was also 


\section{PERCEPTIONS ON CLIMATE CHANGE AND ITS IMPACT ON HUMAN HEALTH}

seen in Bangladesh, where the heat during summers was felt to have increased and rainfall to have decreased, compared to five or ten years earlier (Chaudhary and Bawa, 2011).

Different population groups may have different opinions about climate change (AoyagiUsui, 2008; Combest-Friedman et al., 2012; Haque et al., 2012). We found that those living in non-slum areas and those with higher education levels had heard about climate change significantly more than had people living in slum areas and having less education. Carew-Reid (2008) found that poor people seemed to have less knowledge about the adverse impacts of climate change. For example, poor migrants had more difficulties adapting to climate change as a result of having less knowledge, less support and fewer networks, as well as less experience with floods and storms compared to local residents. Chelsea and Patrick (2012) reported that climate change awareness most strongly depended on the respondents level of education. Heads of households with higher levels of education were more likely to be aware of climate change than those with lower education levels. Another study conducted in Vietnam among poor people suggested that women had less access to information about weather than did men; $60 \%$ of the women had not heard weather forecast information compared to $35.3 \%$ of men, and $35.6 \%$ of women had heard but not understood the information compared to $26.2 \%$ of the men. Limited access to early warning weather information or lack of communications may exacerbate womens difficulties in dealing with climate disaster (Combest-Friedman et al., 2012).

The impact of climate change on human health has been seen in several studies worldwide (Haq et al., 2013; Mai Kien et al., 2010). In this study, people reported that they more easily became ill now than some years ago, although most of the symptoms and diseases mentioned were common, with the exception of the increase in emerging diseases such as the new influenzas, dengue and Japanese encephalitis. This perception might be a result of the findings from another study in Northern Vietnam (Mai Kien et al., 2010), which showed that a warming climate would change seasonal structure, and a warming winter would result in changes in peoples biological rhythm. The results of a study conducted in Australia, Canada, UK, USA and Sweden (Haq et al., 2013) suggested that a warmer climate could have adverse impacts on peoples health which could lead to negative health consequences for vulnerable groups such as old people and those people suffering from cardiac diseases, while 


\section{PERCEPTIONS ON CLIMATE CHANGE AND ITS IMPACT ON HUMAN HEALTH}

a colder climate could lead to increases in coughs/colds, headaches, asthma, pneumonia and especially in re-emerging diseases such as dengue fever or Japanese encephalitis.

A high level of awareness on the links between climate change and human health may help to increase the success of the National Prevention Program on Climate Change. Moreover, understanding the concerns of the people may help policy makers to develop and implement effective and sustainable adaptation measures. However, results from our study also shows that about $10 \%$ of respondent had incorrect ideas, that earthquakes or air pollution were caused by climate change. According to Dr. Nguyen Huu Ninh of the Center for Environment Research Education and Development of Vietnam (personal communication), not many people in Vietnam fully understand about climate change, even among policy makers. Many people are still not aware of the threats of climate change and misunderstand that it is primarily an issue of the environment (Ninh, 2012). Re-framing climate change as a threat to human health can be the principle catalyst for people to change their behavior and increase their support for climate change mitigation and adaptation policies. The public will need to learn to appreciate that climate change is a public health issue too, as it affects peoples health and well-being.

This study does have some limitations. Firstly, the study was conducted among four urban districts in Hanoi, so it may not represent the perceptions elsewhere in Hanoi or Vietnam. Secondly, the issue under study is complicated and difficult to measure. The peoples perceptions on changing health could not be checked against real data on health and possible changes related to climate in this study. Such data could be collected in future to give more precision to the accuracy of the peoples perceptions. There is evidence for an increase in emerging infections such as dengue and Japanese encephalitis as shown in another study of Toan et al. (2013).

\subsection{Conclusion}

Our study revealed that a high proportion of surveyed respondents in the capital city did perceive that climate change is occurring and could mention possible consequences such as heavier rainfall and higher temperatures. The most influential factor on such awareness was 


\section{PERCEPTIONS ON CLIMATE CHANGE AND ITS IMPACT ON HUMAN HEALTH}

the level of education of the respondent; those with higher education had more knowledge about climate change and its impact. Recent climate change has affected Hanoi and may have led to an increase in health problems for its inhabitants.

\subsection{Policy recommendations}

Understanding local perceptions and concerns will assist policy makers to develop effective communication strategies for each location in Vietnam. In this regard, the main lessons emerging from this study are the following:

- More education and awareness-raising is needed for people to appreciate that climate change is a public health issue. A high level of awareness on the links between global environmental change and human health may help to increase the success of the National Prevention Program on Climate Change.

- The Program should make use of community groups as climate change communication channels, especially for the more excluded groups such as women and people with lower levels of education who presently have less awareness of the issues. 
Chapter 9

\section{Discussion and Conclusion}




\section{DISCUSSION AND CONCLUSION}

Epstein (2001) proposed that climate is a key determinant of health and that climate constrains the range of infectious disease, while weather affects the timing and intensity of outbreaks. In this thesis, we investigate the case of dengue fever as a disease that is highly susceptible to different elements of climate change, especially because it is vector-borne.To assess the impact of climate change on dengue transmission, a framework is needed for the public health sector to determine the risks of epidemics in different demographic and geographic regions and to prepare appropriate policies and strategies to mitigate those risks - Although the research for this thesis was conducted only in Hanoi City of Vietnam, a city with a typically sub-tropical climate and considered an endemic region where large dengue outbreaks occur, our findings show potential for application in other similar contexts.

An important objective of this thesis is to provide better understanding of the possible connections between climate change and dengue fever (DF), and to assess the possible impacts of climate change on dengue fever. We contribute to the growing literature on the potential impact of climate change on the transmission of dengue infection. Increased understanding of the relative importance of different variables in the transmission cycles of DF will aid public health planning and policy-making to develop effective strategies to control and prevent this increasingly wide-spread disease. Epidemic forecasting models were developed which may be used directly for the decision-making processes in the surveillance and control of DF. Additionally, the methods developed through this study may have a wider application to other public health problems which have comparable elements in their epidemiology.

Based on the background sketched above, the central question this thesis aims to answer is:

In what different ways might climate change affect dengue fever transmission in Hanoi?

In order to address this question, the following research objectives were identified:

1. To identify any direct and indirect links between climatic variables and DF incidence in Hanoi, Vietnam,

2. To estimate the potential future change of dengue fever pattern in Hanoi,

3. To describe the perceptions on climate change and its impact on human health among 


\section{DISCUSSION AND CONCLUSION}

people in Hanoi.

This chapter revisits these questions based on the findings and main conclusions drawn in this thesis. The chapter closes with concluding remarks to guide future research.

\subsection{Potential effect of climate change on dengue transmission}

A substantial scientific literature has demonstrated that there are at least two ways in which climate change may impact vector-borne diseases such as dengue fever. One mechanism of change is brought about by the direct impact of climate change on the epidemiological triangle of dengue that includes a host, a pathogen and a mosquito vector. For instance, variability in temperature, rainfall, humidity, or storm patterns could result directly in changes in the reproductive rate of the mosquito vector or pathogen, the biting rate of the mosquito vector, or the time the host is exposed to the mosquito vector. A second set of mechanisms is brought about by the indirect impact of climate. In this category, the climate influences one or another parameter important to vector spread or survival, such as the type of agriculture or the species of trees in a forest; this, in turn, changes the relationship between the parasite, vector, and host. Both types of impacts are considered in this thesis; clearly, however, the direct impacts are easier to identify and evaluate than the indirect ones.

Climate variability is postulated to be an important determinant of the non-stationary phase of dengue dynamics (Hales et al., 2002, 1999). In this thesis, two annual (1 year) and sub-annual (6-month) periodicities of DF have been found, which corresponded to a slow increase of DF incidence from the beginning of the year to weeks 22-24 (June), followed by a rapid increase of incidence until weeks 44-46 (November), ending with a sharp decrease in incidence at the end of the rainy season. Wavelet analysis has been demonstrated to be suitable for investigating time series data from non-stationary systems, as is the case for dengue in Hanoi where it is emerging, and for inferring associations within such systems. In Chapter 4, we provide insights into the temporal spread of dengue in Hanoi. We found that all of the climatic variables that we investigated (temperature, rainfall, relative humidities and 


\section{DISCUSSION AND CONCLUSION}

vapor pressure) displayed strong annual periodicities with temperature, rainfall and vapor pressure, which led DF incidence by a consistent delay of 8 to 10 weeks. In addition to this strong annual periodicity, relative humidity displayed a sub-annual periodicity in its effect on DF incidence. The annual periodicity of relative humidity led the annual periodicity of DF incidence by a consistent delay of 18 weeks, whereas the sub-annual periodicity of relative humidity led the sub-annual periodicity of DF by a delay that decreased from 14.30 weeks in 2002 to 5.27 weeks in 2009 at an almost constant rate of 1.13 week per year.

Although we found a very strong correlation between DF and relative humidity, we warn against over-interpretation of this correlation in terms of biological causation. Relative humidity is a variable that depends on both absolute humidity (or rainfall) and temperature but these two variables are most likely not perfectly correlated. Thus, instead of looking for a mechanistic link between DF incidence and relative humidity, we recommended in this thesis to look for two links: (i) one between DF incidence and absolute humidity and (ii) one between DF incidence and temperature, possibly accounting for a potential interactions between the two climatic variables. Furthermore, climatic factors should be considered within the context of the socio-demographic, economic and immunological determinants that contribute to the spread of dengue. These factors are reviewed in Chapters 5, 6 and 7 .

Environmental factors play an essential role in the incidence and prevalence of DF. In Chapter 5, we found that people living near bodies of stagnant water, like ponds, lakes and rivers or open sewers, or near favorable mosquito breeding places like garbage collection points, had higher rates of morbidity. Hanoi is known as a city of ponds and lakes, where water covers approximately 756 hectares and each 0.5 square kilometer of water surface equals one square kilometer of land area. The hydraulic conditions of Hanoi, which correspond to its topographical and climatic features, fall into two main categories: the flooding season and the dry season. The flooding season coincides with the rainy season, lasting from July to October. Flooding reaches its peak in August, bringing with it $70 \%$ to $75 \%$ of the total amount of rainfall of the year (Thang et al., 2010). Moreover, climate change has altered patterns of precipitation, and it is believed to have increased the frequency and intensity of floods in many areas of Hanoi (Bich et al., 2011). Therefore, with a large area of natural water surfaces available and increasing rainfall, an annual dengue epidemic is almost unavoidable 


\section{DISCUSSION AND CONCLUSION}

(Nga, 1992). This risk is confirmed in Chapter 6 where we report that the number of dengue cases per $\mathrm{km}^{2}$ within a zone of $100 \mathrm{~m}$ around water bodies is higher than further away.

Chapter 6 and Chapter 7 presented information about dengue fever hot spot areas in Hanoi. The areas that recorded the highest number of space-time clusters were Dong Da, Hoang Mai and Thanh Xuan with expansion to the north-western wards of Hanoi, where a higher population is concentrated. A relationship between population density and dengue incidence has also been reported in many studies elsewhere, both inside and outside the South East Asia region that includes Vietnam. For example, a report from Malaysia stated that most of the dengue cases were found in the densely populated commercial areas, near construction sites, which were located at topographically low land areas with surrounding high values of land surface temperature. The epidemic normally occurred in these types of areas after days of heavy rainfall followed by high temperature. In Chapter 6, we also report that there were more dengue cases living within a zone of $50 \mathrm{~m}$ around the main roads than further away from them. Indeed, spatial clustering of disease is almost inevitable because human populations live in spatial clusters rather than in a random distribution through space. Furthermore, the results described in Chapters 6 and 7 showed that dengue fever had high spatial autocorrelation within different time periods. It was closely related to the topography of the environment, and villages that were closer together tended to have more similar baseline incidence rates. The Aedes mosquito generally has a short flight range so is unlikely to spread dengue over large distances (Vazquez-Prokopec et al., 2009).

All of these results taken together provide a basis for the understanding of current and future dengue transmission in the urban setting of Hanoi which is likely to be applicable elsewhere in the country and in the region. Application of predictive models can help to use this information to predict disease transmission in the future. A better understanding of factors affecting dengue transmission will support better strategies and plans for prevention and control of the disease. 


\section{DISCUSSION AND CONCLUSION}

\subsection{Potential for change in dengue fever patterns}

The challenge for predicting dengue transmission is how best to model future climate at a regional and/or local level. As discussed in Chapter 2, different scenarios are possible for climate change in general and for Hanoi in particular. Depending on which will actually develop, the predictions for future patterns of dengue will also differ in their accuracy. It is also important to consider which climate variables are the best predictors of dengue distribution. The results described in this thesis suggest that temperature, rainfall, vapor pressure and relative humidity are the significant contributors, which precede the increase in dengue cases by 8 to 10 weeks.

Statistical modeling based on climate data, using methods such as cross correlations, Poisson, logistic and multivariate regression, SARIMA-time series and wavelet time series, has been used frequently in the past years. Such models aim to analyze the impacts of climate on dengue and to study the feasibility of forecasting dengue incidence weeks or months in advance. For example, Pinto et al. (2011) in Singapore, used a Poisson regression model and found that for every $2-10^{\circ} \mathrm{C}$ of variation of the maximum temperature, dengue cases increased by $22.2-184.6 \%$. For the minimum temperature, this increasing percentage was $26.1-230.3 \%$. Similarly, Hu et al. (2010) in Queensland, Australia, applied a Bayesian spatial conditional autoregressive model to identify the impact of climatic, social and ecological factors on dengue. They suggested that a $6 \%$ increase in locally acquired dengue was observed in association with a $1-\mathrm{mm}$ increase in average monthly rainfall and $1^{\circ} \mathrm{C}$ increase in average monthly maximum temperature. In Vietnam, another study conducted recently in Hanoi by Minh An and Joacim (2014), revealed that whenever there was rainfall, an appropriate temperature ranging from $15^{\circ} \mathrm{C}$ to $30^{\circ} \mathrm{C}$, and $\mathrm{DF}$ cases, these three factors would interact together to precede risks of explosion of DF cases by 1 to 2 months. Their results suggested that any time when there are sporadic DF cases and then rainfall during warm weather, the risk of a DF outbreak should be taken into account.

In Vietnam, the estimation from the National Centre for Hydro-Meteorological Forecasting showed that the average temperature in Hanoi has the tendency to rise over time. According to the high emission scenario (A2), the yearly temperature in 2020 in Hanoi should 


\section{DISCUSSION AND CONCLUSION}

increase by about $0.7^{\circ} \mathrm{C}$, or by $0.5^{\circ} \mathrm{C}$ according to medium (B2) and low emission scenarios (B1). In 2030, this increase according to high, medium and low emission scenarios would be $0.9^{\circ} \mathrm{C}, 0.8^{\circ} \mathrm{C}$ and $0.7^{\circ} \mathrm{C}$ respectively. Similarly, it is estimated that, in 2020 , the increase in average yearly rainfall in Hanoi will be about 1.4\% (high emission scenario); $1.3 \%$ (medium emission scenario); and $1.2 \%$ (low emission scenario). In 2030, this increase according to high, medium and low emission scenarios would be $2.1 \%, 1.9 \%$ and $1.8 \%$ respectively. A2 characterizes high population growth, slow economic development and technological changes. B1 characterizes a similar population growth but with rapid economic changes. B2 characterizes a medium population and economic growth with localized solutions to economic, social and environmental sustainability.

For each of these scenarios, the increases in temperature and rainfall could be expected to lead to a continued increase in the number of cases and expansion of the geographical range of dengue fever in Vietnam. Our results demonstrated the strong influence of these two factors on the occurrence of dengue fever and its transmission. Our data also revealed a decreasing lag period between temperature and rainfall and the appearance of dengue infections in recent years. The localization of the largest number of cases to sites near roads and bodies of water points to a recommendation to preventive medicine services to focus control activities in these areas, to reduce the transmission. Unless the policy-makers and planners increase the attention paid to the effects of climate change on health problems such as dengue, the risk of larger epidemics will increase.

\subsubsection{Climate change and human health: public perceptions}

Concepts on climate change and its impacts on human health were primarily addressed in Chapter 8. According to forecasts from the Vietnam National Committee on Climate Change, in the next 40-50 years Hanoi will be severely influenced by climate change. The most obvious consequence of the situation would be land subsidence and the gradual encroachment of the sea. However, an estimated 1 to $4 \mathrm{~m}$ subsidence was currently found for the ground level (VGP, 2011). Boateng (2012) assessed the potential vulnerability of Vietnam's coast to climate change and found that the Red River delta, where the capital city, 


\section{DISCUSSION AND CONCLUSION}

Hanoi, is located, is under severe threat of flooding. In fact, it is observed that there is an increasing trend toward extremes of precipitation and temperature in Hanoi. For example, long and heavy rainfall during the last days of October and the first week of November 2008 resulted in the most devastating flood (90 points inundated locally with an average depth of $0.3 \mathrm{~m}-0.8 \mathrm{~m}$ ) since 1973 (Bich et al., 2011). These occurrences of extreme weather such as storms, floods, deep cold and long heat waves have also been witnessed by the majority of our respondents in Hanoi, as described in Chapter 8. Such abnormal and extreme weather contributes to the confirmation of IPCC (2014) predictions about global occurrence of climate change.

Based on our results from a study using both quantitative and qualitative approaches, and getting opinions from different stakeholders, we stated in Chapter 8 that there is a community awareness of the relationship between climate change and human health. Now, people feel that they become ill more easily than some years ago and that most of the symptoms and diseases are very much related to the season. For example, the most common symptoms reported in relation to hot weather were headache, fatigue, and dizziness, while hypertension and other cardiovascular diseases were thought to have become more common. The symptoms most often mentioned in relation to cold weather were cough and fever, while common diseases were pneumonia, influenza and emerging infectious diseases such as dengue fever or Japanese encephalitis. The exceptional increase of these emerging diseases was explained by proposing that a warming climate would change seasonal structure, and a warming winter would result in changes in people's biological rhythm (Haq et al., 2013; Mai Kien et al., 2010). Therefore, a warmer climate could have adverse impacts on people's health, which could lead to negative health consequences especially for vulnerable groups.

In the case of dengue fever, climate change was seen clearly to have an influence on its transmission. As mentioned in our reports, the warmer temperature and heavier rainfall created favorable conditions for breeding of dengue mosquitoes, leading to the occurrence of dengue fever throughout the year. This statement made by community members was also noted by international scientists, pointing out that climate change is a likely factor for the alterations in both spatial and temporal dynamics of dengue fever around the world. For example, at the current time, dengue fever is occurring in some European countries, 


\section{DISCUSSION AND CONCLUSION}

where before the disease was not endemic (Bouzid et al., 2013; Hunter, 2003). According to Bouzid and colleagues, the increased risk of dengue fever in Europe is related to all climate parameters, which were statistically significant in a highly non-linear way. Rogers and colleagues (2014) showed that most European cities combining warmer temperatures and higher population density are highly suitable for dengue transmission (Rogers and Randolph, 2006). This thesis provides more evidence on the climate-disease relationship. A better understanding of the people's concerns about complex links between climate change and human health, from a local to a global scale, is key to future sustainable development and effective health protection.

\subsubsection{Future perspectives}

Many uncertainties remain regarding the health impacts of climate change on dengue fever. A major challenge for the assessment of future health impacts is the evolution of complex risk factor patterns over time. It is therefore important to elaborate on some of the potential factors that drive dengue activity, as well as the global strategic direction to address the expansion of dengue as a public health problem. This thesis contributes to the understanding of different aspects of the burden of dengue disease, its epidemiology and disease transmission, for a better understanding of dengue fever epidemiology in Hanoi, Vietnam. As described above, one result is a clear warning to the health sector that continued expansion of dengue transmission areas and increasing numbers of cases in existing disease foci can be expected. The health sector needs to prepare for that.

However, in a complex epidemiological setting, transmission dynamics involve interactions among people, dengue viruses, vectors and ecosystems, where biotic and abiotic determinants have both direct and indirect influences on transmission, our models cannot account for all possible variables. Additional research would be needed using more precise data from different sources to consider additional factors such as the social changes that result from economic development but also from climate change.

Furthermore, our analysis on the links between climatic variables and DF incidence in Hanoi raises a number of questions of general interest on the relationships between climate 


\section{DISCUSSION AND CONCLUSION}

and infectious disease epidemiology. Because of its highly seasonal climate, its important population size and density, and its dengue epidemiological transition (currently in a phase of emergence), Hanoi appears to be an ideal situation to test hypotheses about interaction between serotypes. This is an issue both poorly understood and potentially of high relevance for vaccine development. Further investigations on dengue in Hanoi call for additional entomological and immunological data, as well as for continued theoretical developments. 


\section{Bibliography}

Abdul Aziz, A.-A. and Abdullah Rusli, H. I. (2012). Towards the Development of K-Map Model in Visualizing the Dengue Hotspot Using Venn Diagram and AHP Technique. International Journal of Software Engineering, 2(1):7-13.

Adams, B. and Boots, M. (2006). Modelling the relationship between antibody-dependent enhancement and immunological distance with application to dengue. Journal of Theoretical Biology, 242(2):337-346.

Andraud, M., Hens, N., Marais, C., and Beutels, P. (2012). Dynamic Epidemiological Models for Dengue Transmission: A Systematic Review of Structural Approaches. PLoS ONE, $7(11): \mathrm{e} 49085$.

Aoyagi-Usui, M. (2008). An analysis of the effective factors for promoting proenvironmental actions from the information gain and social capital point of view. Rev Environ Econ Policy Stud, 1:37-50.

Arcari, P., Tapper, N., and Pfueller, S. (2007). Regional variability in relationships between climate and dengue/DHF in Indonesia. Singapore Journal of Tropical Geography, $28(3): 251-272$.

Bailey, T. C. and Gatrell, A. C. (1995). Interactive Spatial Data Analysis. Addison Wesley Longman, Harlow, Essex.

Banu, S., Hu, W., Hurst, C., and Tong, S. (2011). Dengue transmission in the Asia-Pacific region: impact of climate change and socio-environmental factors. Tropical Medicine $\mathcal{G}$ 
International Health, 16(5):598-607.

Barbazan, P., Guiserix, M., Boonyuan, W., Tuntaprasart, W., Pontier, D., and Gonzalez, J.-P. (2010). Modelling the effect of temperature on transmission of dengue. Medical and Veterinary Entomology, 24(1):66-73.

Be, P. (2005). Evaluation on knowledge, attitude and practice of people about dengue fever in two communes of An Giang province, Vietnam in 2004. Journal of Preventive Medicine, (3):56-64.

Be, T. T., Sinh, B. T., Miller, F., (Sumernet), S. M. R. N., and 2010-213, S. S. f. i. u. C. N. Y. Y. Q. (2007). Literature analysis : challenges to sustainable development in the Mekong Delta : regional and national policy issues and research needs. Sustainable Mekong Research Network (Sumernet), Bangkok.

Beckett, C. G., Kosasih, H., Faisal, I., null Nurhayati, Tan, R., Widjaja, S., Listiyaningsih, E., Ma'roef, C., Wuryadi, S., Bangs, M. J., Samsi, T. K., Yuwono, D., Hayes, C. G., and Porter, K. R. (2005). Early detection of dengue infections using cluster sampling around index cases. The American Journal of Tropical Medicine and Hygiene, 72(6):777-782.

Berrang-Ford, L., Ford, J., and Patterson, J. (2011). Are we adapting to climate change? Global Environmental Change, 21:25-33.

Bhatt, S., PW, G., OJ, B., JP, M., AW, F., CL, M., and et Al (2013). The global distribution and burden of dengue. Nature, 496:504-507.

Bich, T. H., Quang, L. N., Thanh Ha, L. T., Duc Hanh, T. T., and Guha-Sapir, D. (2011). Impacts of flood on health: epidemiologic evidence from Hanoi, Vietnam. Global Health Action, 4(00).

Boateng, I. (2012). An application of GIS and coastal geomorphology for large scale assessment of coastal erosion and management: a case study of Ghana. Journal of Coastal Conservation, 16(3):383-397.

Bouzid, M., Hooper, L., and Hunter, P. R. (2013). The Effectiveness of Public Health Interventions to Reduce the Health Impact of Climate Change: A Systematic Review of 
Systematic Reviews. PLoS ONE, 8(4):e62041.

Boyce, C. and Neale, P. (2006). Conducting in-depth Interviews: A Guide for Designing and Conducting In-Depth Interviews. Pathfinder International Tool Series.

Braga, C., Luna, C. F., Martelli, C. M., de Souza, W. V., Cordeiro, M. T., Alexander, N., de Albuquerque, M. d. F. P. M. a., Júnior, J. C. S., and Marques, E. T. (2010). Seroprevalence and risk factors for dengue infection in socio-economically distinct areas of Recife, Brazil. Acta Tropica, 113(3):234-240.

Bultó, P. L. O., Rodríguez, A. P., Valencia, A. R., Vega, N. L., Gonzalez, M. D., and Carrera, A. P. (2006). Assessment of human health vulnerability to climate variability and change in Cuba. Environmental Health Perspectives, 114(12):1942-1949.

Burke, D. S., Nisalak, A., Johnson, D. E., and Scott, R. M. (1988). A prospective study of dengue infections in Bangkok. The American Journal of Tropical Medicine and Hygiene, $38(1): 172-180$.

Carew-Reid, J. (2008). Rapid Assessment of the Extent and Impact of Sea Level Rise in Viet Nam. International Centre for Environmental Management.

Cazelles, B., Chavez, M., C, M. G., F, G. J., and Hales, S. (2007). Time-dependent spectral analysis of epidemiological time-series with wavelets. Journal of the Royal Society, Interface / the Royal Society, 4:625-636.

Cazelles, B., Chavez, M., McMichael, A. J., and Hales, S. (2005). Nonstationary Influence of El Niño on the Synchronous Dengue Epidemics in Thailand. PLoS Medicine, 2(4):e106.

Cazelles, B. and Hales, S. (2006). Infectious Diseases, Climate Influences, and Nonstationarity. PLoS Medicine, 3(8):e328.

Chadee, D. D., Shivnauth, B., Rawlins, S. C., and Chen, A. A. (2007). Climate, mosquito indices and the epidemiology of dengue fever in Trinidad (2002-2004). Annals of Tropical Medicine and Parasitology, 101(1):69-77.

Chadee, D. D., Williams, F. L. R., and Kitron, U. D. (2005). Impact of vector control on a dengue fever outbreak in Trinidad, West Indies, in 1998. Tropical medicine 8 international 
health: TM \& IH, 10(8):748-754.

Chan, N. Y., Ebi, K. L., Smith, F., Wilson, T. F., and Smith, A. E. (1999). An integrated assessment framework for climate change and infectious diseases. Environ Health Perspect, 107(5):329-337.

Chang, M. S., Hii, J., Buttner, P., and Mansoor, F. (1997). Changes in abundance and behaviour of vector mosquitoes induced by land use during the development of an oil palm plantation in Sarawak. Transactions of the Royal Society of Tropical Medicine and Hygiene, 91(4):382-386.

Chaterji, S., Allen, J. C., Chow, A., Leo, Y.-S., and Ooi, E.-E. (2011). Evaluation of the NS1 rapid test and the WHO dengue classification schemes for use as bedside diagnosis of acute dengue fever in adults. The American Journal of Tropical Medicine and Hygiene, $84(2): 224-228$.

Chaudhary, P. and Bawa, K. S. (2011). Local perceptions of climate change validated by scientific evidence in the Himalayas. Biology Letters, 7(5):767-770.

Chen, L. H. and Wilson, M. E. (2004). Transmission of Dengue Virus without a Mosquito Vector: Nosocomial Mucocutaneous Transmission and Other Routes of Transmission. Clinical Infectious Diseases, 39(6):e56-e60.

Chen, S.-C. and Hsieh, M.-H. (2012). Modeling the transmission dynamics of dengue fever: implications of temperature effects. The Science of the Total Environment, 431:385-391.

Chowell, G., Torre, C. A., Munayco-Escate, C., Suárez-Ognio, L., López-Cruz, R., Hyman, J. M., and Castillo-Chavez, C. (2008). Spatial and temporal dynamics of dengue fever in Peru: 1994-2006. Epidemiology and Infection, 136(12):1667-1677.

Combest-Friedman, C., Christie, P., and Miles, E. (2012). Household perceptions of coastal hazards and climate change in the Central Philippines. Journal of Environmental Management, 112:137-148.

Costello, A., Abbas, M., Allen, A., Ball, S., Bell, S., Bellamy, R., and et (2009). Managing the health effects of climate change. The Lancet, 373(9676):1693-1733. 
Côte, J., Salmela, J. H., Baria, A., and Russell, S. (1993). Applied Research Organizing and Interpreting Unstructured Qualitative Data. Sport Psychol, 10:247-260.

Coudeville, L. and Garnett, G. P. (2012). Transmission Dynamics of the Four Dengue Serotypes in Southern Vietnam and the Potential Impact of Vaccination. PLoS ONE, $7(12): \mathrm{e} 51244$.

Cox, P. M., Betts, R. A., Bunton, C., Essery, R. L. H., Rowntree, P. R., and Smith, J. (1999). The impact of new land surface physics on the GCM simulation of climate and climate sensitivity. Climate Dyn., 15:183-203.

Cuong, H., Hien, N., Duong, T., Phong, T., Cam, N., and et Al (2011). Quantifying the emergence of dengue in Hanoi, Vietnam: 1998-2009. PLoS neglected tropical diseases, 5:e1322.

DANIDA (2005). Climate change in Vietnam; Country report; Hanoi, Vietnam. DANIDA.

Dasgupta, S., Laplante, B., Meisner, C., Wheeler, D., and Yan, J. (2009). The impact of sea level rise on developing countries: a comparative analysis. Climatic Change, 93(3-4):379388.

Depradine, C. and Lovell, E. (2004). Climatological variables and the incidence of Dengue fever in Barbados. International Journal of Environmental Health Research, 14(6):429441.

Descloux, E., Mangeas, M., Menkes, C. E., Lengaigne, M., Leroy, A., Tehei, T., Guillaumot, L., Teurlai, M., Gourinat, A.-C., Benzler, J., Pfannstiel, A., Grangeon, J.-P., Degallier, N., and De Lamballerie, X. (2012). Climate-Based Models for Understanding and Forecasting Dengue Epidemics. PLoS Neglected Tropical Diseases, 6(2):e1470.

Dolan, A. H. and Walker, I. J. (2006). Understanding Vulnerability of Coastal Communities to Climate Change Related Risks. Journal of Coastal Research, pages 1316-1323.

Dung, N. T. and Cam, N. N. (2005). Dengue fever/dengue hemorrhagic fever in Hanoi 2003. Journal of Vietnam Preventive Medicine, (1):73-77.

Dunlap, R. E. and McCright, A. M. (2008). A widening gap: republican and democratic 


\section{BIBLIOGRAPHY}

views on climate change. Environment, 50(5):26-35.

Duoc, V. T., Dung, T. C., Phong, T. V., and Chen, C. C. (2011). Dispersal of Aedes aegypti and Aedes albopictus in urban endemic areas of Vietnam. Journal of Medical Research of Vietnam, (9):30-34.

Duong, L. (2009). Climate change: Ha Giang, Vietnam adapting to climate change.

Earnest, A., Tan, S. B., and Wilder-Smith, A. (2012). Meteorological factors and El Niño Southern Oscillation are independently associated with dengue infections. Epidemiology and Infection, 140(07):1244-1251.

Eastin, M. D., Delmelle, E., Casas, I., Wexler, J., and Self, C. (2014). Intra- and Interseasonal Autoregressive Prediction of Dengue Outbreaks Using Local Weather and Regional Climate for a Tropical Environment in Colombia. American Journal of Tropical Medicine and Hygiene, 91(3):598-610.

Ebi, K. L., Hartman, J., Chan, N., Mcconnell, J., Schlesinger, M., and Weyant, J. (2005). Climate Suitability for Stable Malaria Transmission in Zimbabwe Under Different Climate Change Scenarios. Climatic Change, 73(3):375-393.

Ebi, K. L., Lewis, N. D., and Corvalan, C. (2006). Climate Variability and Change and their Potential Health Effects in Small Island States: Information for Adaptation Planning in the Health Sector. Environmental Health Perspectives.

Emch, M., Feldacker, C., Yunus, M., Streatfield, P. K., DinhThiem, V., Canh, D. G., and Ali, M. (2008). Local environmental predictors of cholera in Bangladesh and Vietnam. The American Journal of Tropical Medicine and Hygiene, 78(5):823-832.

Epstein, P. R. (2001). Climate change and emerging infectious diseases. Microbes Infect, $3: 747-754$.

Epstein, P. R. (2005). Climate Change and Human Health. New England Journal of Medicine, 353(14):1433-1436.

Few, R. and Tran, P. G. (2010). Climatic hazards, health risk and response in Vietnam: Case studies on social dimensions of vulnerability. Global Environmental Change, 20(3):529- 
538.

Ford, J. and Furgal, C. (2009). Climate change impacts, adaptation and vulnerability in the Arctic. Polar Research, 28(1):1-9.

Ford, J. D. (2012). Indigenous health and climate change. American Journal of Public Health, 102(7):1260-1266.

Friel, S., Marmot, M., McMichael, A., Kjellstrom, T., and Vger, D. (2008). Global health equity and climate stabilisation - need for a common agenda. The L, 372(9650):1677-1683.

Gesler, W. (1986). The uses of spatial analysis in medical geography: a review. Social Science \& Medicine (1982), 23(10):963-973.

Getis, A., Morrison, A. C., Gray, K., and Scott, T. W. (2003). Characteristics of the spatial pattern of the dengue vector, Aedes aegypti, in Iquitos, Peru. The American Journal of Tropical Medicine and Hygiene, 69(5):494-505.

Gharbi, M., Quenel, P., Gustave, J., Cassadou, S., Ruche, G., Girdary, L., and Marrama, L. (2011). Time series analysis of dengue incidence in Guadeloupe, French West Indies: Forecasting models using climate variables as predictors. BMC Infectious Diseases, 11(1):166.

Gonzalez, L. E. and da Silveira, P. (1997). The people's attitudes towards global environmental phenomena: a case study. Clim Res, (9):95-100.

Gouhier, T. and Grinsted, A. (2013). biwavelet: Conduct univariate and bivariate wavelet analyses.

GSO (2008). Statistical Yearbook of Viet Nam. Hanoi: Statistical Publishing House.

GSO (2012). Statistical Yearbook of Viet Nam. Hanoi: Statistical Publishing House.

Gubler, D. J. (1998). Dengue and dengue hemorrhagic fever. Clinical Microbiology Reviews, 11(3):480-496.

Gubler, D. J. (2011). Dengue, Urbanization and Globalization: The Unholy Trinity of the 21st Century. Tropical Medicine and Health, 39(4 Suppl):3-11.

Gubler, D. J., Reiter, P., Ebi, K. L., Yap, W., Nasci, R., and Patz, J. A. (2001). Climate 


\section{BIBLIOGRAPHY}

variability and change in the United States: potential impacts on vector- and rodent-borne diseases. Environmental Health Perspectives, 109(Suppl 2):223-233.

Ha, D. Q. and Huan, T. Q. (2000). Dengue activity in Vietnam and its control program, 1997-1998. Dengue Bulletin, 21:35-43.

Ha, D. Q. and Ninh, T. U. (2000). Virological surveillance of dengue haemorrhagic fever in Vietnam, 1987-1999. Dengue Bull, 24:18-23.

Hajat, S. and Kosatky, T. (2010). Heat-related mortality: a review and exploration of heterogeneity. Epidemiol. Community Health, 64:753760.

Hales, S., de Wet, N., Maindonald, J., and Woodward, A. (2002). Potential effect of population and climate changes on global distribution of dengue fever: an empirical model. Lancet (London, England), 360(9336):830-834.

Hales, S., N, d. W., Maindonald, J., and Woodward, A. (1999). El Nino and the dynamics of vectorborne disease transmission. Environmental health perspectives, 107:99-102.

Halmar, H. R. and Peter, R. (2011). Early Warning System for Dengue Hemorrhagic Fever (DHF) Epidemics in Makassar. Journal Matematika Dan Sains, 16(2).

Haq, G., Snell, C., Gutman, G., and Brown, D. (2013). Global ageing and environmental change. Attitudes, risks and opportunities; Project report 2013. Stockholm Environment Institute.

Haque, M. A., Yamamoto, S., Malik, A., and Sauerborn, R. (2012). Households' perception of climate change and human health risks: A community perspective. Environmental Health, 11(1):1.

Hartley, L. M., Donnelly, C. A., and Garnett, G. P. (2002). The seasonal pattern of dengue in endemic areas: mathematical models of mechanisms. Transactions of the Royal Society of Tropical Medicine and Hygiene, 96(4):387-397.

Hay, S. I., Myers, M. F., Maynard, N., and Rogers, D. (2002). From remote sensing to relevant sensing in human health. Photogrammetric Engineering and Remote Sensing, 68(2):109. 
Herring, S. C., Hoerling, M. P., Peterson, T. C., Stott, P. A., and et (2014). Explaining Extreme Events of 2013 from a Climate Perspective. Bull. Amer. Meteor. Soc, 95(9):S1S96.

Heukelbach, J., de Oliveira, F. A., Kerr-Pontes, L. R., and Feldmeier, H. (2001). Risk factors associated with an outbreak of dengue fever in a favela in Fortaleza, north-east Brazil. Tropical medicine $\&$ international health: TM $\&$ IH, 6(8):635-642.

Higa, Y., Thi Yen, N., Kawada, H., Hai Son, T., Thuy Hoa, N., and Takagi, M. (2010). Geographic Distribution of Aedes aegypti and Aedes albopictus Collected from Used Tires in Vietnam. Journal of the American Mosquito Control Association, 26(1):1-9.

Honório, N. A., Castro, M. G., de Barros, F. S. M., Magalhães, M. d. A. F. M., and Sabroza, P. C. (2009). The spatial distribution of Aedes aegypti and Aedes albopictus in a transition zone, Rio de Janeiro, Brazil. Cadernos de Saúde Pública, 25(6):1203-1214.

Hosmer, D. W. and Lemeshow, S. (2000). Applied Logistic Regression. Wiley, New York.

Hsieh, Y. H. and Chen, C. W. S. (2009). Turning points, reproduction number, and impact of climatological events for multi-wave dengue outbreaks. Tropical medicine $\mathcal{E}$ international health: TM \& IH, 14(6):628-638.

Hsieh, Y.-H., de Arazoza, H., and Lounes, R. (2013). Temporal trends and regional variability of 2001-2002 multiwave DENV-3 epidemic in Havana City: did Hurricane Michelle contribute to its severity? Tropical medicine $\&$ international health: TM $\mathscr{G} I H, 18(7): 830-$ 838.

Hsueh, Y.-H., Lee, J., and Beltz, L. (2012). Spatio-temporal patterns of dengue fever cases in Kaoshiung City, Taiwan, 20032008. Applied Geography, 34:587-594.

Hu, W., Clements, A., Williams, G., and Tong, S. (2010). Dengue fever and El Nino/Southern Oscillation in Queensland, Australia: a time series predictive model. Occupational and environmental medicine, 67:307-311.

Hu, W., Clements, A., Williams, G., and Tong, S. (2011). Spatial analysis of notified dengue fever infections. Epidemiology and Infection, 139(3):391-399. 
Hu, W., Nicholls, N., Lindsay, M., Dale, P., McMichael, A. J., Mackenzie, J. S., and Tong, S. (2004). Development of a predictive model for ross river virus disease in Brisbane, Australia. The American Journal of Tropical Medicine and Hygiene, 71(2):129-137.

Hunter, P. R. (2003). Climate change and waterborne and vector-borne disease. Journal of Applied Microbiology, 94(s1):37-46.

Huong, L. T. T. and et Al. (2009). Knowledge, attitude and practice on dengue prevention among residences in Binh Thanh commune, Thanh Binh District, Dong Thap province, Vietnam. Journal of Preventive Medicine, 11:36-42.

Huynen, M. M. and Martens, P. (2008). Linkages among globalization, human rights, and health. In: Soskolne, C., Westra, L., Kotze, L., Mackey, B., Rees, W., et al. (Eds.) Sustaining life on earth: environmental and human health through global governance. Lanham: Lexington Books.

Huynen, M. M., Martens, P., Schram, D., Weijenberg, M. P., and Kunst, A. E. (2001). The impact of heat waves and cold spells on mortality rates in the Dutch population. Environmental Health Perspectives, 109(5):463-470.

IFAD, I. F. f. A. D. (2014). Comprehensive environment and climate change assessment in Viet Nam. International Fund for Agricultural Development.

IFPRI (2011). Viet Nam statistics and climate change impacts. A presentation by the International Food Policy Research Institute. Technical report, 5 May 2011. Washington, D.C.

IPCC (2007). Climate change 2007: Synthesis Report. Contribution of Working groups I, II and III to the Fourth Assessment Report of the Intergovernmental Panel on Climate Change. (104).

IPCC (2014). Summary for Policymakers: Climate change 2014. Impacts, Adaptation, and Vulnerability. Technical report.

Itrat, A., Khan, A., Javaid, S., Kamal, M., Khan, H., Javed, S., Kalia, S., Khan, A. H., Sethi, M. I., and Jehan, I. (2008). Knowledge, Awareness and Practices Regarding Dengue Fever 
among the Adult Population of Dengue Hit Cosmopolitan. PLoS ONE, 3(7):e2620.

Jacobs, A. F. G., Heusinkveld, B. H., and Paaijmans, K. P. (2005). Shallow water temperatures and malarial mosquitoes. Bulletin of the American Meteorological Society, 86(1):2526.

Janssen, M. A. and Martens, W. J. (1997). Modeling malaria as a complex adaptive system. Artificial Life, 3(3):213-236.

Johansson, M. A., Dominici, F., and Glass, G. E. (2009). Local and Global Effects of Climate on Dengue Transmission in Puerto Rico. PLoS Neglected Tropical Diseases, 3(2):e382.

Kearney, M., Porter, W. P., Williams, C., Ritchie, S., and Hoffmann, A. A. (2009). Integrating biophysical models and evolutionary theory to predict climatic impacts on species ranges: the dengue mosquito $\mathrm{i}_{i} \mathrm{~A}_{\text {Aedes aegypti }} / \mathrm{i}_{i}$ in Australia. Functional Ecology, $23(3): 528-538$.

Keeling, M. and Rohani, P. (2008). Modeling infectious diseases in humans and animals, volume 38. Princeton University Press, 1 edition.

Khasnis, A. A. and Nettleman, M. D. (2005). Global warming and infectious disease. Archives of Medical Research, 36(6):689-696.

Knox, T. B., Yen, N. T., Nam, V. S., Gatton, M. L., Kay, B. H., and Ryan, P. A. (2007). Critical Evaluation of Quantitative Sampling Methods for Aedes aegypti (Diptera: Culicidae) Immatures in Water Storage Containers in Vietnam. Journal of Medical Entomology, 44(2):192-204.

Knudsen, A. B. (1995). Global distribution and continuing spread of Aedes albopictus. Parassitologia, 37(2-3):91-97.

Koenraadt, C. J. M., Tuiten, W., Sithiprasasna, R., Kijchalao, U., Jones, J. W., and Scott, T. W. (2006). Dengue knowledge and practices and their impact on Aedes aegypti populations in Kamphaeng Phet, Thailand. The American Journal of Tropical Medicine and Hygiene, 74(4):692-700.

Kovats, R. S., Bouma, M. J., Hajat, S., Worrall, E., and Haines, A. (2003). El Niño and 
health. Lancet, 362(9394):1481-1489.

Kroeger, A. and Nathan, M. B. (2006). Dengue: setting the global research agenda. Lancet, 368(9554):2193-2195.

Kulldorff, M. (1997). A spatial scan statistic. Communications in Statistics - Theory and Methods, 26(6):1481-1496.

Kulldorff, M. (2006). SaTScan user guide. 7:19-20.

Lamers, M., Liggett, D., and Amelung, B. (2012). Strategic challenges of tourism development and governance in Antarctica: taking stock and moving forward. Polar Research, $31(0)$.

Lawson, A. B. and Williams, F. L. R. (2001). An Introductory Guide to Disease Mapping. Chichester, UK: John Wiley $\mathcal{G}$ Son.

Leiserowitz, A. A. (2005). American risk perceptions: is climate change dangerous? Risk Analysis: An Official Publication of the Society for Risk Analysis, 25(6):1433-1442.

Lin, C.-F., Wan, S.-W., Cheng, H.-J., Lei, H.-Y., and Lin, Y.-S. (2006). Autoimmune pathogenesis in dengue virus infection. Viral Immunology, 19(2):127-132.

Lowe, R., Bailey, T., Stephenson, D., Graham, R., and et $\mathrm{Al}$ (2008). Climate-based dengue predictions for Brazil. https://www.stat.aau.at/Tagungen/statgis/2009/StatGIS2009 Lowe $_{1} . p d f$.

Lu, L., Lin, H., Tian, L., Yang, W., Sun, J., and Liu, Q. (2009). Time series analysis of dengue fever and weather in Guangzhou, China. BMC Public Health, 9(1):395.

Mai Kien, T., Thi Tuyet Hanh, T., Duc Cuong, H., and Shaw, R. (2010). Chapter 20 Identifying linkages between rates and distributions of malaria, water-born diseases and influenza with climate variability and climate change in Vietnam. In Shaw, R., Pulhin, J. M., and Jacqueline Pereira, J., editors, Climate Change Adaptation and Disaster Risk Reduction: An Asian Perspective, volume 5, pages 417-449. Emerald Group Publishing Limited.

Martens, P. (1997). Vulnerability of human population health to climate change: state of 


\section{BIBLIOGRAPHY}

knowledge and future research directions. Change, (38):5-8.

Martens, P. (2002). Climate change effect on vector-borne diseases: evidence or absence of evidence. Environment, Risques ES Santé, (1):9-12.

Martens, P. and Huynen, M. (2001). Will global climate change reduce thermal stress in the Netherlands? Epidemiology (Cambridge, Mass.), 12(6):753-754.

Martens, W., Jetten, T., Rotmans, J., and Niessen, L. (1995a). Climate change and vectorborne diseases. Global Environmental Change, 5(3):195-209.

Martens, W. J. (1998). Climate change, thermal stress and mortality changes. Social Science E Medicine (1982), 46(3):331-344.

Martens, W. J., Niessen, L. W., Rotmans, J., Jetten, T. H., and McMichael, A. J. (1995b). Potential impact of global climate change on malaria risk. Environmental Health Perspectives, 103(5):458-464.

McConnell, K. J. and Gubler, D. J. (2003). Guidelines on the cost-effectiveness of larval control programs to reduce dengue transmission in Puerto Rico. Revista Panamericana De Salud Pública = Pan American Journal of Public Health, 14(1):9-16.

McElwee, P. (2010). The social dimensions of adaptation of climate change in Vietnam. Development and climate change discussion paper. Washington, DC: World Bank., 17.

McMichael, A. J. and Butler, C. D. (2006). Emerging health issues: the widening challenge for population health promotion. Health Promotion International, 21 Suppl 1:15-24.

McMichael, A. J., Woodruff, R. E., and Hales, S. (2006). Climate change and human health: present and future risks. The Lancet, 367(9513):859-869.

Mihov, C., Tuong, C., and Tuong, H. (1959). Dengue epidemic in Hanoi. Folia Med, 1:169-73.

Minh An, D. T. and Joacim, R. (2014). Epidemiology of dengue fever in Hanoi from 2002 to 2010 and its meteorological determinants. Global Health Action, 7(0).

MOH (1999). Guidelines on the surveillance, diagnosis, and treatment of dengue hemorrhagic fever. Vietnam Ministry of Health. 
MOH (2006). Guidelines for Surveillance, Prevention and Control of Dengue and Dengue Haemorrhagic Fever. Vietnam Ministry of Health.

MOH (2007). National strategy on preventive medicine and master plan on health care system development. Vietnam Ministry of Health.

MOH (2010). Guidance on declaration, communication, and reporting infectious deseases. Vietnam Ministry of Health, Circula 48/2010/TT-BYT.

MONRE (2009). Climate Change, Sea Level Rise Scenarios for Vietnam. Ministry of Natural Resources and Environment.

MONRE/PEP/UNDP (2008). Climate change adaptation and the poor. A study of four coastal communities in Ha Tinh and Ninh Thuan provinces of Vietnam. Ministry of Natural Resources and Environment.

Moore, A., Seng, S. B., and Chong, A. K. (2005). Geostatistical modelling, analysis and mapping of epidemiology of Dengue Fever in Johor State, Malaysia.

Murray, N. E. A., Quam, M. B., and Wilder-Smith, A. (2013). Epidemiology of dengue: past, present and future prospects. Clinical Epidemiology, 5:299-309.

Myers, S. S. and Patz, J. A. (2009). Emerging Threats to Human Health from Global Environmental Change. Annual Review of Environment and Resources, 34(1):223-252.

Nagao, Y., Thavara, U., Chitnumsup, P., Tawatsin, A., Chansang, C., and CampbellLendrum, D. (2003). Climatic and social risk factors for Aedes infestation in rural Thailand. Tropical medicine \& international health: TM \& IH, 8(7):650-659.

Nga, N. T. (1992). Epidemiology of dengue fever/dengue hemorrhagic fever in Hai Ba Trung District, Hanoi City. Master thesis of Hanoi Medical University, Vietnam.

Nguyen, L. A. P., Clements, A. C. A., Jeffery, J. A. L., Yen, N. T., Nam, V. S., Vaughan, G., Shinkfield, R., Kutcher, S. C., Gatton, M. L., Kay, B. H., and Ryan, P. A. (2011). Abundance and prevalence of Aedes aegypti immatures and relationships with household water storage in rural areas in southern Viet Nam. International Health, 3(2):115-125.

Nguyen, N. D. (1999). Knowledge, attitude and practice on dengue/dengue hemorrhagic 


\section{BIBLIOGRAPHY}

fever prevention among mothers in Ho Chi Minh City, Vietnam. Journal of Ho Chi Minh City's Medical Practice, (2):119-124.

NIHE (2009). Final Report on evaluation of communicable diseases surveillance system in Vietnam 2008. Hanoi: National Institute of Hygiene and Epidemiology.

Ninh, N. (2012). Coping with climate change for a sustainability development.

Nishiura, H. (2006). Mathematical and statistical analyses of the spread of dengue. Dengue Bulletin, (30).

NTP (May, 2008). National Target Program to Respond to Climate Change (Unofficial translation of Vietnamese draft version of 13/5/2008). Hanoi.

Ooi, E.-E. and Gubler, D. J. (2009). Dengue in Southeast Asia: epidemiological characteristics and strategic challenges in disease prevention. Cadernos De Saúde Pública, 25 Suppl 1:S115-124.

Patton, M. Q. (2002). Qualitative research \& education methods. Thousand Oaks, CA: Sage.

Patz, J., McGeehin, M., Bernard, S., Ebi, K., Epstein, P., Grambsch, A., and et Al (2000). The potential health impacts of climate variability and change for the United States: executive summary of the report of the health sector of the U.S. National Assessment. Environ Health Perspect, 108(4):367-76.

Patz, J. A., Martens, W. J., Focks, D. A., and Jetten, T. H. (1998). Dengue fever epidemic potential as projected by general circulation models of global climate change. Environmental Health Perspectives, 106(3):147-153.

Paz-Soldan, V. A., Plasai, V., Morrison, A. C., Rios-Lopez, E. J., Guedez-Gonzales, S., Grieco, J. P., Mundal, K., Chareonviriyaphap, T., and Achee, N. L. (2011). Initial Assessment of the Acceptability of a Push-Pull Aedes aegypti Control Strategy in Iquitos, Peru and Kanchanaburi, Thailand. American Journal of Tropical Medicine and Hygiene, $84(2): 208-217$.

Pham, H. V., Doan, H. T. M., Phan, T. T. T., and Tran Minh, N. N. (2011). Ecological 


\section{BIBLIOGRAPHY}

factors associated with dengue fever in a central highlands Province, Vietnam. $B M C$ Infectious Diseases, 11(1):172.

Pinto, E., Coelho, M., Oliver, L., and Massad, E. (2011). The influence of climate variables on dengue in Singapore. International Journal of Environmental Health Research, 21(6):415426.

Preet, R., Nilsson, M., Schumann, B., and Evengå rd, B. (2010). The gender perspective in climate change and global health. Global Health Action, 3(0).

Promprou, S., Jaroensutasinee, M., and Jaroensutasinee, K. (2005a). Climatic factors affecting dengue haemorrhagic fever incidence in Southern Thailand. Dengue Bulletin, 29:41-48.

Promprou, S., Jaroensutasinee, M., and Jaroensutasinee, K. (2005b). Impact of Climatic Factors on Dengue Haemorrhagic Fever Incidence in Southern Thailand. Walailak J Sci \& Tech, 2:59-70.

RCoreTeam (2012). R: A Language and Environment for Statistical Computing R. Foundation for Statistical Computing Vienna, Austria.

Recker, M., Blyuss, K. B., Simmons, C. P., Hien, T. T., Wills, B., Farrar, J., and Gupta, S. (2009). Immunological serotype interactions and their effect on the epidemiological pattern of dengue. Proceedings of the Royal Society B: Biological Sciences, 276(1667):2541-2548.

Reiter, P. (2001). Climate change and mosquito-borne disease. Environmental Health Perspectives, 109(Suppl 1):141-161.

Rogers, D. J. and Randolph, S. E. (2006). Climate change and vector-borne diseases. Advances in Parasitology, 62:345-381.

Rotmans, J. (1998). Methods for IA: The challenges and opportunities ahead. Environmental Modeling $\&$ Assessment, 3(3):155-179.

Rueda, L. M., Patel, K. J., Axtell, R. C., and Stinner, R. E. (1990). Temperature-dependent development and survival rates of Culex quinquefasciatus and Aedes aegypti (Diptera: Culicidae). Journal of Medical Entomology, 27(5):892-898.

Schreiber, K. V. (2001). An investigation of relationships between climate and dengue using 
a water budgeting technique. International Journal of Biometeorology, 45(2):81-89.

Semenza, J. C. and Menne, B. (2009). Climate change and infectious diseases in Europe. The Lancet. Infectious Diseases, 9(6):365-375.

Shaman, J. and Kohn, M. (2009). Absolute humidity modulates influenza survival, transmission, and seasonality. Proceedings of the National Academy of Sciences of the United States of America, 106(9):3243-8.

Shepard, D. S., Undurraga, E. A., and Halasa, Y. A. (2013). Economic and Disease Burden of Dengue in Southeast Asia. PLoS Neglected Tropical Diseases, 7(2).

Silverman, D. (2000). Doing qualitative research: A practical handbook. London, SAGE.

Simoes, T. C., Codeco, C. T., Nobre, A. A., and Eiras, A. E. (2013). Modeling the Non-Stationary Climate Dependent Temporal Dynamics of Aedes aegypti. PLoS ONE, 8(8):e64773.

Small, J., Goetz, S. J., and Hay, S. I. (2003). Climatic suitability for malaria transmission in Africa, 19111995. Proceedings of the National Academy of Sciences, 100(26):15341-15345.

Soper, H. E. (1929). The Interpretation of Periodicity in Disease Prevalence. Journal of the Royal Statistical Society, 92(1):34.

Sutherst, R. W. (2004). Global Change and Human Vulnerability to Vector-Borne Diseases. Clinical Microbiology Reviews, 17(1):136-173.

Syed, M., Saleem, T., Syeda, U.-R., Habib, M., Zahid, R., Bashir, A., Rabbani, M., Khalid, M., Iqbal, A., Rao, E. Z., null Shujja-ur Rehman, and Saleem, S. (2010). Knowledge, attitudes and practices regarding dengue fever among adults of high and low socioeconomic groups. JPMA. The Journal of the Pakistan Medical Association, 60(3):243-247.

Tanser, F. C., Sharp, B., and le Sueur, D. (2003). Potential effect of climate change on malaria transmission in Africa. The Lancet, 362(9398):1792-1798.

Teixeira, M. d. G., Barreto, M. L., Costa, M. d. C. a. N., Ferreira, L. D. A., Vasconcelos, P. F. C., and Cairncross, S. (2002). Dynamics of dengue virus circulation: a silent epidemic in a complex urban area. Tropical medicine $\mathcal{E}$ international health: TM $\mathcal{E}$ IH, 7(9):757-762. 
Thai, K. T. D. and Anders, K. L. (2011). The role of climate variability and change in the transmission dynamics and geographic distribution of dengue. Experimental Biology and Medicine (Maywood, N.J.), 236(8):944-954.

Thai, K. T. D., Cazelles, B., Nguyen, N. V., Vo, L. T., Boni, M. F., Farrar, J., Simmons, C. P., van Doorn, H. R., and de Vries, P. J. (2010). Dengue Dynamics in Binh Thuan Province, Southern Vietnam: Periodicity, Synchronicity and Climate Variability. PLoS Neglected Tropical Diseases, 4(7):e747.

Thang, N., Cuong, H., Mau, N., and Kien, T. (2010). Development of Climate Change scenarios for Vietnam by precis model. Vietnam Institute of Meteorology, Hydrology and Environment.

Thornes, J. E. (2002). IPCC, 2001: Climate change 2001: impacts, adaptation and vulnerability, Contribution of Working Group II to the Third Assessment Report of the Intergovernmental Panel on Climate Change, edited by J. J. McCarthy, O. F. Canziani, N. A. Leary, D. J. Dokken and K. S. White (eds). Cambridge University Press, Cambridge, UK, and New York, USA, 2001. No. of pages: 1032. Price: 34.95, ISBN 0-521-015006 (paperback), ISBN 0-521-80768-9 (hardback). International Journal of Climatology, 22(10):1285-1286.

Thu, H. M., Aye, K. M., and Thein, S. (1998). The effect of temperature and humidity on dengue virus propagation in Aedes aegypti mosquitos. The Southeast Asian Journal of Tropical Medicine and Public Health, 29(2):280-284.

Tien, N. T. K., Ha, D. Q., Tien, T. K., and Quang, L. C. (1999). Predictive indicators for forecasting epidemic of dengue/dengue haemorrhagic fever through epidemiological, virological and entomological surveillance. Dengue Bull, 23:34-39.

Tien, N. T. K., Tuan, N. N. A., Tuan, K. M., Toan, N. T., and Quang, L. C. (2001). Epidemiological analysis of deaths associated with dengue haemorrhagic fever in southern Vietnam in 19992000. Dengue Bulletin, (25):28-32.

Tipayamongkholgul, M., Fang, C.-T., Klinchan, S., Liu, C.-M., and King, C.-C. (2009). Effects of the El Niño-Southern Oscillation on dengue epidemics in Thailand, 1996-2005. 
BMC Public Health, 9(1):422.

Toan, D. T. T., Hoat, L. N., Hu, W., Wright, P., and Martens, P. (2014). Risk factors associated with an outbreak of dengue fever/dengue haemorrhagic fever in Hanoi, Vietnam. Epidemiology and Infection, 143(8):1594-1598.

Toan, D. T. T., Hu, W., Thai, P. Q., Hoat, L. N., Wright, P., and Martens, P. (2013). Hot spot detection and spatio-temporal dispersion of dengue fever in Hanoi, Vietnam. Global Health Action, 6(0).

Torrence, C. and Compo, G. P. (1998). A practical guide to wavelet analysis. Bull. Am. Meteorol. Soc., 79:61-78.

Tseng, W.-C., Chen, C.-C., Chang, C.-C., and Chu, Y.-H. (2009). Estimating the economic impacts of climate change on infectious diseases: a case study on dengue fever in Taiwan. Climatic Change, 92(1-2):123-140.

Tun-Lin, W., Burkot, T. R., and Kay, B. H. (2000). Effects of temperature and larval diet on development rates and survival of the dengue vector Aedes aegypti in north Queensland, Australia. Medical and Veterinary Entomology, 14(1):31-37.

Tun-Lin, W., Kay, B. H., and Barnes, A. (1995). The Premise Condition Index: a tool for streamlining surveys of Aedes aegypti. The American Journal of Tropical Medicine and Hygiene, 53(6):591-594.

Umor, S. M., Mokhta, M. B., Surip, N., and Ahmad, A. (2008). Generating a dengue risk map (DRM) based on environmental factors using remote sensing and GIS technologies. Asian Conference on Remote Sensing.

Valkering, P., Amelung, B., Van der Brugge, R., and Rotmans, J. (2006). More PuzzleSolving for Policy. Integrated Assessment from Theory to Practice. Maastricht: ICIS \& EFIEA.

Van Asselt, M., Mellors, J., Rijkens-Klomp, N., Greeuw, S., Molendijk, K., Jelle Beers, P., and Van Notten, P. (2001). Building Blocks for Participation in Integrated Assessment: A Review of Participatory Methods. ICIS, Maastricht. 
van Asselt Marjolein, B. A. and Rijkens-Klomp, N. (2002). A look in the mirror: reflection on participation in Integrated Assessment from a methodological perspective. Global Environmental Change, 12(3):167-184.

Van Benthem, B. H. B., Vanwambeke, S. O., Khantikul, N., Burghoorn-Maas, C., Panart, K., Oskam, L., Lambin, E. F., and Somboon, P. (2005). Spatial patterns of and risk factors for seropositivity for dengue infection. The American Journal of Tropical Medicine and Hygiene, 72(2):201-208.

van der Sluijs, J. P. (2001). Integrated assessment models and the participatory challenge.

The case of climate change, in: Dunn, W. N., Hisschemoller, M., Hoppe, R. and Ravetz, J. R. (Eds.), Knowledge, Power, and Participation in Environmental Policy Analysis. Transaction Pub, (Special Issue Policy Studies Review Annual):317-348.

Vanwambeke, S. O., Lambin, E. F., Eichhorn, M. P., Flasse, S. P., Harbach, R. E., Oskam, L., Somboon, P., van Beers, S., van Benthem, B. H. B., Walton, C., and Butlin, R. K. (2007). Impact of Land-use Change on Dengue and Malaria in Northern Thailand. EcoHealth, $4(1): 37-51$.

Vazquez-Prokopec, G., Stoddard, S., Paz-Soldan, V., Morrison, A., Elder, J., Kochel, T., Scott, T., and Kitron, U. (2009). Usefulness of commercially available GPS data-loggers for tracking human movement and exposure to dengue virus. Int J Health Geogr, 8(68).

VGP (2011). The National strategy on climate change was issued by Prime Minister Nguyen Tan Dung in Decision 2139/QD-TTg on December 05, 2011. Vietnam Goverment Portal. Vu, S. N., Nguyen, T. Y., Tran, V. P., Truong, U. N., Le, Q. M., Le, V. L., Le, T. N., Bektas, A., Briscombe, A., Aaskov, J. G., Ryan, P. A., and Kay, B. H. (2005). Elimination of dengue by community programs using Mesocyclops(Copepoda) against Aedes aegypti in central Vietnam. The American Journal of Tropical Medicine and Hygiene, 72(1):67-73.

Wallace, J. M. and Hobbs, P. V. (2006). Atmospheric Science, An Introductory Survey. Academic New York, 2nd Ed.

Watts, D. M., Burke, D. S., Harrison, B. A., Whitmire, R. E., and Nisalak, A. (1987). Effect 


\section{BIBLIOGRAPHY}

of temperature on the vector efficiency of Aedes aegypti for dengue 2 virus. The American Journal of Tropical Medicine and Hygiene, 36(1):143-152.

Watts, G. (2010). Water for people: climate change and water availability. chapter 4 in fung f., lopez a. and new m. (eds) modelling the impact of climate change on water resources. John Wiley.

WB (2010). Viet Nam Economics of adaptation to climate change. Technical report, Washington, D.C.: World Bank Group.

WHO (1997). Clinical diagnosis. In Dengue haemorrhagic fever: diagnosis, treatment, prevention and control. World Health Organization, Geneva.

WHO (2008). Experts propose simplified system of dengue classification. TDR news.

WHO, W. H. O. (2003). Climate change and human health - risks and responses. World Health Organization, Geneva.

WHO, W. H. O. (2009). Protecting health from climate change: global research priorities. World Health Organization, Geneva, Switzerland.

Wongkoon, S., Jaroensutasinee, M., and Jaroensutasinee, K. (2011). Climatic variability and dengue virus transmission in Chiang Rai, Thailand. Biomedica, 27:5-13.

Wu, P.-C., Guo, H.-R., Lung, S.-C., Lin, C.-Y., and Su, H.-J. (2007). Weather as an effective predictor for occurrence of dengue fever in Taiwan. Acta Tropica, 103(1):50-57.

Yanai, H., Thiem, V., Matsubayashi, T., Huong, V., Suzuki, M., and et (2007). The Khanh Hoa Health Project: characterization of study population and field site development for clinical epidemiological research on emerging and re-emerging infectious diseases. Tropical Medicine and Health, 35:61-65.

Yi, B., Zhang, Z., Xu, D., Xi, Y., Fu, J., Luo, J., Yuan, M., Liu, S., and Zuo, Z. (2003). Relationship of dengue fever epidemic to Aedes density changed by climate factors in Guangdong Province. Wei Sheng Yan Jiu, 32(2):152-154. 


\section{Summary}

The Intergovernmental Panel on Climate Change has warned that a changing climate could affect public health, which includes diseases like dengue fever. In recent decades, both the incidence and the geographical distribution of dengue have increased dramatically. Chapter 1 in this thesis is an introduction to the current knowledge on climate change, both globally and in Vietnam. A small increase in annual mean temperature could signify moderate variation in daily weather that in turn has an impact on the ecosystems and biodiversity of the sub-tropical regions where this research was done. In Chapter 1, we also mention the climate-health linkage and describe that relationship in the case of dengue fever, in which climate affects the dengue virus and vector populations in both direct and indirect ways. The thesis contributes to the knowledge base on the potential impact of climate change on the transmission of dengue infection, which is needed for formulation of effective policies and strategies.

The central question of our thesis was: In what different ways might climate change affect dengue fever transmission in the specific context of Hanoi?

In answering the research question, a mixture of theoretical and empirical material was used. The theoretical part in Chapter 2 of the thesis offers a literature review that provides more detailed background information on dengue fever and climate change, with specific reference to the city of Hanoi. The 2014 report from IPCC predicted that climate change may lead to more frequent and intense natural disasters such as typhoons, floods, droughts and saline water intrusion. The IPCCs Fourth Assessment characterized Vietnam as a natural disaster hotspot, ranking $7^{\text {th }}$ globally on economic risk, $9^{\text {th }}$ on land area and population exposed, and $22^{\text {nd }}$ on mortality from multiple hazards. One key issue in this last point 
is the growing burden of dengue fever in the world and in Vietnam. Dengue is not one disease entity, but a spectrum of diseases caused by four serotypes of dengue virus, which is the most prevalent arthropod-borne virus affecting human in the tropics and sub-tropics. Because it is transmitted by a mosquito vector, this infection is quite susceptible to variations in the weather. Dengue fever is a growing public health problem in Vietnam that has been recognized as a major cause of mortality and morbidity, ranking among the top ten communicable diseases in terms of overall health burden. The current surveillance and control program on the dengue vector in Vietnam was described. In fact, the relation between dengue fever and climate change has been mentioned in quite a few studies around the world, but has still not received much attention from the Government of Vietnam.

Integrated Assessment provides an overarching research philosophy to collect all the information from different sources on one topic, as presented in Chapter 3. In this thesis, the principles of IA have been realized by combining knowledge from different disciplines and research methods, from both social and natural sciences. Using quantitative methods, we addressed the direct and indirect impact of climate change on dengue fever. To explore the perceptions of people in Hanoi about climate change and perceptions of the impact of climate change on health, we applied participatory, qualitative methods. We also identified a number of limitations related to the methodologies used. The major limitation for the quantitative approaches was the issue of how to control for the uncertainties, especially concerning the quality of the available data. For the qualitative work, the main limitation was the difficulty of getting all stakeholders actively involved to provide their opinions and understanding during group discussions.

Chapters 4 to 7 report on investigations to identify conditions resulting from climate change that directly or indirectly affect the transmission of dengue fever. First we checked for direct links between climate change and dengue fever. By applied wavelet analysis method, we could detect and measure the seasonality of DF and investigate its direct links to climatic variables. In Chapter 4 we 
describe how all three climatic variables investigated - temperature, rainfall and vapor pressure - showed strong seasonality and consistency over time. Meanwhile the relative humidity showed both strong seasonality and a sub-annual periodicity with a decreased in lag time before dengue appears, through time. These two peaks per year were also detected when we calculated the dengue fever reproductive ratio and interpreted it in terms of mosquito population dynamics and immunological interactions between the different dengue serotypes in the human compartment.

To demonstrate an indirect link between climate change and dengue, we also explored other risk factors such as environmental or population factors. In Chapter 5, we explored the risk factors associated with DF in patients admitted to hospitals in Hanoi. We looked at several independent variables including the demographic characteristics of the patients, such as age, education, occupation, and the variables related to their housing conditions as potential risk factors for dengue. This integrated analysis of the eco-social determinants of transmission risk helped to identify the key risk factors for dengue in Hanoi having indirect links with climate change. Factors significantly associated with occurrence of dengue were: living in rented housing, living near open sewers, and living in a house discharging sewage directly into ponds.

Together with the findings from Chapter 5, the contribution of environmental factors to the expansion of dengue transmission was also revealed in Chapter 6. Proximity to transportation arteries such as major roadways or to rivers was found to contribute to the geographical spread of dengue. People living within a zone of 50m around Hanois main roads and within a zone of 50 to $100 \mathrm{~m}$ around water bodies (where the mosquito larvae become adults) tended to have a higher risk of infection than people living outside these zones. Not only areas with low land topography, but also areas with high population density were associated with outbreaks of dengue fever. In the densely populated urban areas, chances of contact between man and mosquito increase. In Hanoi we found that the six central districts in the North and the Center of the city, with the highest 
population density and highest immigration rates, witnessed more dengue cases than other areas.

Six of the 14 districts of Hanoi, where a higher population is concentrated, recorded the highest numbers of space-time clusters of dengue infection, lasting 29 days and within a radius of $1,000 \mathrm{~m}$. This result was described in Chapter 7, which also revealed that the geographical distribution of dengue in Hanoi has expanded in recent years. Moreover, in Chapter 7, we found a significant positive spatial autocorrelation of dengue incidence through time periods, which means that incidence rates per commune can be seen as clusters. By applying spatial autocorrelation methods, we noticed that the closer a commune is to another commune, the larger the chance that its incidence rate has approximately the same value as its neighbor.

The evidence about occurrence of climate change and its impact on human health, especially on dengue fever transmission, has been shown using different methods in all of these chapters. In Chapter 8, we address the perception of the inhabitants of Hanoi about climate change and its possible effects on health. According to the majority of our respondents, climate change is occurring; they interpreted it in the form of changes in rainfall, temperature, or strong wind during the rainy season. Illness among family members, especially children, was thought to have increased during both summer and winter compared to five years earlier. The most common symptoms reported during hot weather were headaches, fatigue, and dizziness; hypertension and other cardiovascular diseases were also reported. During cold weather, people reported experiencing cough, fever, and influenza, as well as pneumonia and emerging infectious diseases such as dengue and Japanese encephalitis. Appreciating the complex relations between climate change and human health as well as awareness of peoples concerns are keys for the policy-makers and planners working towards future sustainable development and effective health protection.

Chapter 9 recapitulates the main conclusions drawn from the results in this $\mathrm{PhD}$ 
thesis, provides a brief reflection on the methods and findings, and offers an outlook to future work. The main research question underlying this study asks in what different ways climate change might affect dengue fever transmission in Hanoi. We answer this question based on the results of investigations into three aspects: potential effects of climate change on dengue transmission; potential changes in dengue fever patterns; and public perceptions on climate change and human health. We demonstrate that climate can affect the size and geographical spread of the dengue epidemic through both direct and indirect effects on the virus, the vector and the population. As the climate continues to change, we can expect continued spread of the disease in Vietnam. This thesis contributes to the understanding of the burden of dengue disease, its epidemiology and disease transmission, for a better understanding of dengue fever epidemiology as climate change goes on in Hanoi, Vietnam. The findings from detailed investigations in this large urban area in which dengue is still an emerging disease will be relevant for other areas especially in Southeast Asia with a similar context. 


\section{Samenvatting}

Het 'Intergovernmental Panel on Climate Change (IPCC) waarschuwt dat een veranderend klimaat de volksgezondheid kan bëınvloeden, onder meer door ziekten als knokkelkoorts. In de afgelopen decennia is zowel de incidentie als de geografische verspreiding van knokkelkoorts aanzienlijk toegenomen. Hoofdstuk 1 van dit proefschrift bevat een inleiding op de huidige kennis van de klimaatverandering, zowel wereldwijd als in Vietnam. Een kleine stijging van de jaarlijkse gemiddelde temperatuur kan tot een gematigde variatie in de dagelijkse weersomstandigheden leiden, die op haar beurt effect heeft op de ecosystemen en biodiversiteit van de subtropische regio's waar dit onderzoek werd verricht. In hoofdstuk 1 noemen we tevens het verband tussen klimaat en gezondheid en beschrijven we deze relatie voor knokkelkoorts: het klimaat heeft zowel direct als indirect invloed op het knokkelkoortsvirus en de vectorpopulaties. Het proefschrift levert een bijdrage aan de kennis van de mogelijke effecten van klimaatverandering op de overdracht van een knokkelkoortsinfectie. Dergelijke kennis is nodig met het oog op de formulering van doeltreffend beleid en dito strategiëen.

De centrale vraag van ons proefschrift luidde: op welke verschillende manieren kan klimaatverandering de overdracht van knokkelkoorts in de specifieke context van Hanoi bënvloeden?

Bij het beantwoorden van de onderzoeksvraag werd een combinatie van theoretisch en empirisch materiaal gebruikt. Het theoretische deel van hoofdstuk 2 van het proefschrift bevat een literatuurstudie waarin meer gedetailleerde achtergrondinformatie over knokkelkoorts en klimaatverandering wordt verschaft, met een specifieke verwijzing naar de stad Hanoi. In het IPCC-rapport van 2014 werd voorspeld dat klimaatverandering tot frequentere en intensere natuurrampen als tyfoons, overstromingen, droogtes en insijpeling van zout water kan leiden. In het vierde rapport van de IPCC werd Vietnam als een "kritiek gebied voor natuurrampen" gekenschetst. Dat land stond op de 7 de plaats wat betreft economische risico's, op de 9de plaats wat betreft de blootgestelde oppervlakte en 
populatie en op de 22ste plaats wat betreft de sterfte als gevolg van diverse gevaren. Belangrijk bij dit laatste punt is de groeiende last van knokkelkoorts in de wereld en in Vietnam. Knokkelkoorts vormt niet één ziekteentiteit, maar een spectrum van ziekten die wordt veroorzaakt door vier serotypen van het knokkelkoortsvirus, het meest voorkomende door muggen overgebracht virus met nadelige gevolgen voor mensen in tropische en subtropische gebieden. Omdat deze infectie via vectormuggen wordt overgedragen, is zij behoorlijk vatbaar voor weersverschillen. Knokkelkoorts is een groeiend volksgezondheidsprobleem in Vietnam dat als een belangrijke oorzaak van sterfte en ziekte is erkend. Knokkelkoorts staat in dat land in de top tien van overdraagbare ziekten wat de algehele gezondheidslast betreft. Er is een lopend programma voor toezicht op en bestrijding van de knokkelkoortsvector in Vietnam. Echter, alhoewel de relatie tussen knokkelkoorts en klimaatverandering in een flink aantal studies over de hele wereld wordt vermeld, schenkt de Vietnamese regering daar nog steeds weinig aandacht aan.

Een gëntegreerde benadering (Integrated Assessment (IA)) biedt een overkoepelende onderzoeksfilosofie om alle informatie over één onderwerp uit diverse bronnen te verzamelen. Dit komt aan de orde in hoofdstuk 3. In dit proefschrift zijn de beginselen van een IA toegepast door kennis uit verschillende disciplines en onderzoeksmethoden van zowel sociale als natuurwetenschappen te combineren. Met behulp van kwantitatieve methoden hebben we het directe en indirecte effect van klimaatverandering op knokkelkoorts onder de loep genomen. Om de opvattingen van mensen in Hanoi over klimaatverandering en de opvattingen over het effect van klimaatverandering op de gezondheid te onderzoeken, hebben we participatieve, kwalitatieve methoden toegepast. Ook hebben we een aantal beperkingen vastgesteld die met de gehanteerde methodieken verband houden. De belangrijkste beperking bij de kwantitatieve benaderingen was de vraag hoe voor onzekerheden moest worden gecontroleerd, met name wat de kwaliteit van de beschikbare gegevens betreft. Ten aanzien van de kwalitatieve analyses was de grootste beperking de moeilijkheid om alle belanghebbenden actief betrokken te krijgen zodat zij hun standpunten en inzichten tijdens groepsdiscussies kenbaar zouden maken.

In de hoofdstukken $4 \mathrm{t} / \mathrm{m} 7$ wordt verslag gedaan van onderzoek naar omstandigheden als gevolg van klimaatverandering die de overdracht van 
knokkelkoorts direct of indirect bënvloeden. Eerst hebben we gekeken naar directe verbanden tussen klimaatverandering en knokkelkoorts. Met behulp van een wavelet-analysemethode konden we opsporen en meten hoe seizoensgebonden knokkelkoorts is en de directe verbanden tussen deze ziekte en klimaatvariabelen onderzoeken. In hoofdstuk 4 beschrijven we hoe alle drie onderzochte klimaatvariabelen - temperatuur, regenval en dampdruk in de loop van de tijd een sterke seizoensgebondenheid en consistentie vertoonden. Ondertussen liet de relatieve luchtvochtigheid zowel een sterke seizoensgebondenheid als een subjaarlijkse periodiciteit zien met in de loop van de tijd een kortere aanlooptijd. Deze twee pieken in het jaar werden eveneens gedetecteerd toen we de reproductieratio van knokkelkoorts berekenden en deze interpreteerden in termen van muggenpopulatiedynamiek en immunologische interacties tussen de verschillende serotypen van knokkelkoorts in het humane compartiment.

Om een indirect verband tussen klimaatverandering en knokkelkoorts aan te tonen, hebben we ook onderzoek verricht naar andere risicofactoren, zoals milieu- of populatiefactoren. In hoofdstuk 5 onderzoeken we de risicofactoren die met knokkelkoorts worden geassocieerd, bij patiënten in ziekenhuizen in Hanoi. We hebben gekeken naar diverse onafhankelijke variabelen waaronder de demografische kenmerken van de patiënten, zoals leeftijd, opleiding en beroep, en naar de variabelen die verband houden met hun woonomstandigheden als potentiële risicofactoren voor knokkelkoorts. Mede dankzij deze gëntegreerde analyse van de ecosociale determinanten van het overdrachtsrisico konden de belangrijkste risicofactoren voor knokkelkoorts in Hanoi die indirect met klimaatverandering in verband staan, worden vastgesteld. Factoren die significant met het voorkomen van knokkelkoorts werden geassocieerd, waren: wonen in een huurhuis, wonen bij een open riool en wonen in een huis waarvan het rioolwater rechtstreeks in een vijver wordt geloosd.

De bijdrage van milieufactoren aan de toegenomen overdracht van knokkelkoorts komt niet alleen aan de orde in hoofdstuk 5, maar ook in hoofdstuk 6. Gebleken is dat de nabijheid van verkeersaders zoals grote wegen of van rivieren een bijdrage levert aan de geografische verspreiding van knokkelkoorts. Mensen die binnen een straal van $50 \mathrm{~m}$ rond de hoofdwegen en binnen een straal van 50-100 m rond waterlichamen (waar de mug- 
genlarven volwassen worden) woonden, liepen meer kans besmet te raken dan mensen die buiten deze gebieden woonden. Niet alleen laag liggende gebieden, maar ook gebieden met een hoge bevolkingsdichtheid werden geassocieerd met uitbraken van knokkelkoorts. In de dichtbevolkte stedelijke gebieden is de kans op contact tussen mensen en muggen het grootst. In Hanoi constateerden we dat in de zes centrale districten in het noorden en het centrum van de stad met de hoogste bevolkingsdichtheid en de hoogste immigratiecijfers, meer gevallen van knokkelkoorts voorkwamen dan in andere gebieden.

In zes van de veertien districten van Hanoi, die het dichtstbevolkt zijn, werden de grootste aantallen tijd-ruimteclusters van knokkelkoortsinfectie geregistreerd (29 dagen binnen een straal van $1.000 \mathrm{~m}$ ). Dit resultaat wordt beschreven in hoofdstuk 7, waar ook staat vermeld dat de geografische verspreiding van knokkelkoorts in Hanoi de afgelopen jaren is vergroot. Bovendien hebben we in hoofdstuk 7 vastgesteld dat er sprake is van een significante positieve ruimtelijke autocorrelatie van de incidentie van knokkelkoorts gedurende verschillende tijdsperioden, wat betekent dat de incidentiecijfers per gemeente als clusters kunnen worden beschouwd. Met behulp van ruimtelijke-autocorrelatiemethoden hebben we geconstateerd dat hoe dichter een gemeente bij een andere gemeente ligt, hoe groter de kans is dat haar incidentiecijfer ongeveer dezelfde waarde heeft als dat van de buurgemeente.

Dat het klimaat verandert en dat dit fenomeen invloed heeft op de menselijke gezondheid, vooral op de overdracht van knokkelkoorts, is in al deze hoofdstukken met behulp van diverse methoden aangetoond. In hoofdstuk 8 gaan we in op de vraag hoe de inwoners van Hanoi tegen klimaatverandering en de mogelijke effecten daarvan op de gezondheid aankijken. Volgens de meeste van onze respondenten was er sprake van een veranderd patroon van weersfactoren; dat leidden ze af uit veranderingen in regenval, temperatuur of een krachtige wind tijdens het regenseizoen. Men dacht dat gezinsleden, vooral kinderen, vaker ziek waren dan vijf jaar daarvoor, zowel in de zomer als in de winter. De symptomen bij warm weer die het vaakst werden genoemd, waren hoofdpijn, vermoeidheid en duizeligheid; verder werd melding gemaakt van hoge bloeddruk en harten vaatziekten. Bij koud weer hadden mensen last van hoesten, koorts en griep, en tevens van longontsteking en opkomende infectieziekten 
als knokkelkoorts en Japanse encefalitis. Voor de beleidsmakers en planners die aan duurzame ontwikkeling en doeltreffende bescherming van de gezondheid in de toekomst werken, is het van essentieel belang dat zij inzicht hebben in de complexe relaties tussen klimaatverandering en menselijke gezondheid en zich bewust zijn van de zorgen die mensen bezighouden.

In hoofdstuk 9 worden de belangrijkste conclusies die uit de resultaten van dit proefschrift kunnen worden getrokken, samengevat, met tevens een korte reflectie op de methoden en bevindingen en een vooruitzicht op toekomstige werkzaamheden. De belangrijkste onderzoeksvraag die aan deze studie ten grondslag ligt, is op welke manieren klimaatverandering de overdracht van knokkelkoorts in Hanoi kan bënvloeden. Wij beantwoorden deze vraag op basis van de uitkomsten van onderzoek naar drie aspecten: potentiële effecten van klimaatverandering op de overdracht van knokkelkoorts; potentiële wijzigingen in patronen van knokkelkoorts; en de opvattingen van de bevolking over klimaatverandering en menselijke gezondheid. We tonen aan dat het klimaat invloed kan hebben op de omvang en geografische verspreiding van de knokkelkoortsepidemie via zowel directe als indirecte effecten op het virus, de vector en de populatie. Omdat het klimaat blijft veranderen, kunnen we verwachten dat de verspreiding van de ziekte in Vietnam zal doorgaan. Dit proefschrift helpt meer inzicht te krijgen in de last van knokkelkoorts en het epidemische karakter en de overdracht ervan. Doel is een beter inzicht in het epidemische karakter van knokkelkoorts te verkrijgen, aangezien het klimaat in Hanoi, Vietnam zal blijven veranderen. De bevindingen van gedetailleerd onderzoek in dit grote stedelijke gebied waarin knokkelkoorts nog steeds een opkomende ziekte is, zijn relevant voor andere gebieden binnen een soortgelijke context, met name in Zuidoost-Azië. 


\section{Valorisation}

At the core of the valorisation process is the notion of translation of knowledge into products and services. The topic of our research thesis was the translation of information on the direct or indirect impacts of climate change into the transmission of dengue fever in Hanoi. The thesis consists of five empirical chapters, a theory chapter, a methodology chapter plus the introduction and conclusions chapter.

It is motivated by the observation that even increases in dengue incidence and severity are related to many factors including urbanization, increasing migration from rural to urban areas, mobility of people up and down the city, habitat pollution, floods, and climate factors such as temperature, rainfall, and humidity, little attention on these impacts, especially for the impact of climate change is being given by the Government of Vietnam. The purpose of this study is to contribute to the literature on the relative importance of social and ecological variables in the transmission cycles of DF that will be aid into public health planning and policy making. Based on that, the Vietnamese Government will develop effective strategies to control this increasingly wide-spread disease. Although the research for this thesis was conducted only in Hanoi, a city with a typically sub-tropical climate and considered an endemic region where large dengue outbreaks occur, our findings show potential for application to other similar contexts. Our thesis provided some main messages. A model-based dengue early warning system that uses climatic variables would provide evidence-based or scientific estimation of the risk of dengue in future weeks or months, estimate risk of dengue using a long period of historical data. Using weekly aggregated data of dengue incidence and four climatic variables, we found temperature, 
rainfall, and vapor pressure have strong seasonality, while DF and relative humidity show both strong seasonality and a sub-annual periodicity. Temperature, rainfall and vapor pressure lead DF incidence by a lag of 8-10 weeks, constant through time. Relative humidity leads DF by a constant lag of 18 weeks for the annual cycle and a lag decreasing from 14 to 5 weeks for the sub-annual cycle. The estimation from the Vietnam National Centre for Hydro-Meteorological Forecasting showed that the average temperature in Hanoi has the tendency to rise overtime. According to the high emission scenario (A2), the yearly temperature in 2020 in Hanoi should increase by about $0.7^{\circ} \mathrm{C}$, or by $0.5^{\circ} \mathrm{C}$ according to medium (B2) and low emission scenarios (B1). In 2030, this increase according to high, medium and low emission scenarios would be $0.9^{\circ} \mathrm{C}, 0.8^{\circ} \mathrm{C}$ and $0.7^{\circ} \mathrm{C}$ respectively. Evidence is accumulating from many studies including ours about the relationship between climate change and DF. The statistical numbers estimated from three scenarios in Hanoi have clearly warned about a continued increase of DF epidemics in Hanoi in the future. The rising of the climatic variables and the time distance between climatic variables and DF could be indicators for a warning of increasing risk of dengue cases for the local authorities. The primary audience for our research are policy makers in the public health sector. Therefore, public health authorities in Hanoi should be prepared for controlling the continuing increase of DF in the future. Our thesis also demonstrated that the DF reproductive ratio is increasing through time and displays two clear peaks per year, reflecting the sub-annual periodicity of DF incidence. The results are interpreted in terms of mosquito population dynamics and immunological interactions between the different dengue serotypes in the human compartment. Given its important population size, its strong seasonality and its dengue emergence, Hanoi offers an ideal natural experiment to test hypotheses on dengue serotype interactions, knowledge that is of prime importance for vaccine development.

Climate change should not stand alone, it should be considered within the context of the socio-demographic, economic and immunological determinants that 
contribute to the spread of dengue. The other message in our thesis is about the essential role of environmental factors in the increased incidence and prevalence of DF. We found that people living near bodies of stagnant water, like ponds, lakes and rivers or open sewers, or near favorable mosquito breeding places like garbage collection points, had higher rates of morbidity. We also detected the presence of long term hotspots of DF occurrence in six districts of Hanoi where has higher population density. These findings can provide public health officials with necessary information, thus enabling them to chalk out more effective strategies to control DF. Furthermore, our thesis provides a message to Hanoi Preventive Medicine Center that hotspot analysis for DF should be widely used in DF surveillance, since it can help them to allocate resources to deal with outbreaks more effectively.

Another target group in our research is the community members. As the results revealed that many of them still had incorrect ideas about climate change and little awareness about links between climate change and human health, more effort is needed to raise awareness that climate change is a public health issue. A high level of awareness on the links between global environmental change and human health may help to increase the success of the National Prevention Program on Climate Change. The Program should also make use of community groups as climate change community channels, especially for the more excluded groups such as women and people with lower levels of education who presently have less awareness of the issues.

Finally, the research results are valuable for the research and science community. Our research used real databases from local/national statistics stations to build up statistics model based for Hanoi a typical sub-tropical city. The successful application of a range of methods and tools demonstrated how useful they could be in describing a complex system and identifying key factors where interventions may be possible. Thus our application could show the potential of the proposed model for other similar contexts. 


\section{Acknowledgements}

This research project was realized with funding from the Netherlands Higher Education (NTP) project on Strengtherning teaching and research capacity in preventive medicine in Vietnam.

This dissertation would not have been possible without the support and encouragement of many people.

Special mentions goes to my promotor Prof. Pim Martens and co-promotors Ass.Prof. Luu Ngoc Hoat and Prof. Pamela Wright, for providing me with the opportunity to complete my $\mathrm{PhD}$ thesis at the Maastricht University. At all times throughout the course of my $\mathrm{PhD}$ study, they have maintained diligence, interest and enthusiasm for my research. Their critical and thoughtful comments, guidance, support and advice have helped me a lot in doing this thesis. Dr. Pim, your enthusiasm inspired me and your experience with the theories and models was my guide through this complex field. Dr. Hoat, as my boss you were always ready to give a helping hand and support me through the hard work and difficulties, and keep me motivated to complete the work. Dr. Pamela, you welcomed me to your $\mathrm{PhD}$ house in Amsterdam, where several Vietnamese have written their theses, shared meals, but also shared English lessons and along the way lessons about reading and writing critically for science.

I gratefully acknowledge to my co-authors Wenbiao Hu, Marc Choisy, Bram Oosterbroek and Pham Quang Thai. Their statistical expertise has taught me a lot, and been essential in helping me complete my studies.

Thanks also to my colleagues from Hanoi Preventive Medicine Center and Vietnam National Centre for Hydro Meteorological Forecasting, who helped me to 
finish my field work and provided me with the extensive data sets necessary for my work.

Many thanks to MUNDO projects staff in Maastricht, particularly Mike Robertson, Hennie Sijen and Sjoerd Kusters for your memorial supports in order to make my trips in Maastricht smooth and comfortable. Also in Maastricht I shared with and learned from fellow students, Jane Prieto and her husband Yos and Chang Chiung whose companionship I enjoyed.

Also, many thanks to all staff in the International Centre for Integrated assessment and Sustainable development (ICIS), Maastricht University for welcoming and supporting me, particularly Mrs. Anja van Bogaert and Mrs. Annet Grol for very helpful administrative support.

My thanks are due also to the leaders, teachers and staff of the Institute of Training for Preventive Medicine and Public Health, Hanoi Medical University, where I have been working since the year 2001. Particularly, to Ass.Prof. Le Thi Huong, the Director of the Institute for her trust in me and Dr. Le Thi Thanh Xuan and Dr. Nguyen Thi Thuy Hanh for the way they treated me as their younger sister. I am especially grateful to my colleagues in the Department of Biostatistics and Medical Informatics (Ass.Prof. Luu Ngoc Hoat, Le Van Hoi, Hoang Hai Van, Le Xuan Hung and Dinh Thai Son). Without their support, encouragement and sharing of my duties, I could not have done this $\mathrm{PhD}$ thesis. I am also grateful to my colleagues at the Department of Administrative management (Dr. Le Thi Thanh Xuan, Nguyen Huu Thang, Nguyen Thi Thinh, Nguyen Thuy Hien, Le Thu Huong, Nguyen Xuan Tuan and Nguyen Thi Binh) where I was the Vice Head of Department from 2012. The colleagues always encouraged me and shared my work when I was absent for my study.

My big thanks to other PhD students at NUFFIC project, Ms. Pham Bich Diep, Nguyen Van Anh and Nguyen Phuong Lan for home and street-sharing and supporting me during my time away from home by preparing food and a daily life chat. 
Words can not express how grateful I am to my father, my mother and in particularly my father, who sadly passed away before the publication of this book. Spiritual support also came from all other members of my family, including the in-laws and I would like to thank to them. Finally, but by no means least, special thanks go to my husband and my two children. To my beloved husband, Dr. Nguyen Quoc Ai, I would like to express my thanks for supporting me for everything, and especially I cant thank you enough for encouraging me throughout this experience. To my beloved son Nguyen Duc Lan and daughter Nguyen Do Minh Anh, I would like to express my thanks for being such a good children always cheering me up. They are the most important people in my world and I dedicate this thesis to them. 